Keywords SMECT, weight percent solids

Retention: permanent

\title{
EVALUATION OF A TURBIDITY METER FOR USE AT THE DEFENSE WASTE PROCESSING FACILITY
}

R.N. Mahannah

T.B. Edwards

January 2013

Applied Computational Engineering and Statistics Savannah River National Laboratory

Aiken, SC 29808

This document was prepared in conjunction with work accomplished under Contract No. DE-AC09-08SR22470 with the U.S. Department of Energy.

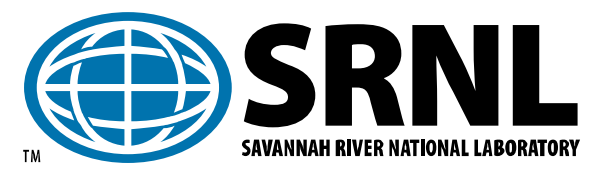


SRNL-STI-2013-00016

Revision 0

\section{DISCLAIMER}

This work was prepared under an agreement with and funded by the U.S. Government. Neither the U.S. Government or its employees, nor any of its contractors, subcontractors or their employees, makes any express or implied: 1. warranty or assumes any legal liability for the accuracy, completeness, or for the use or results of such use of any information, product, or process disclosed; or 2. representation that such use or results of such use would not infringe privately owned rights; or 3. endorsement or recommendation of any specifically identified commercial product, process, or service. Any views and opinions of authors expressed in this work do not necessarily state or reflect those of the United States Government, or its contractors, or subcontractors.

This document was prepared in conjunction with work accomplished under Contract No. DE-AC09-08SR22470 with the U.S. Department of Energy. 
SRNL-STI-2013-00016

Revision 0

\section{REVIEWS AND APPROVALS}

\section{AUTHORS:}

R. N. Mahannah, Waste Services Laboratory

Date

Savannah River Remediation

T. B. Edwards, Applied Computational Engineering and Statistics

Date

Savannah River National Laboratory

\section{TECHNICAL REVIEWER:}

E. P. Shine, Applied Computational Engineering and Statistics Savannah River National Laboratory

Date

Savannah River National Laboratory

\section{APPROVERS:}

P. L. Lee, Manager, Applied Computational Engineering and Statistics Savannah River National Laboratory

C. C. Herman, Manager, Process Technology Programs

Date

Savannah River National Laboratory

R. T. McNew, Manager, Waste Services Laboratory

Date

Savannah River Remediation

E. J. Freed, Manager, Waste Solidification Engineering

Date

Savannah River Remediation 


\section{EXECUTIVE SUMMARY}

Savannah River Remediation's (SRR's) Defense Waste Processing Facility (DWPF) Laboratory currently tests for sludge carry-over into the Recycle Collection Tank (RCT) by evaluating the iron concentration in the Slurry Mix Evaporator Condensate Tank (SMECT) and relating this iron concentration to the amount of sludge solids present. A new method was proposed for detecting the amount of sludge in the SMECT that involves the use of an Optek turbidity sensor. Waste Services Laboratory (WSL) personnel conducted testing on two of these units following a test plan developed by Waste Solidification Engineering (WSE). Both Optek units (SN64217 and SN65164) use sensor model AF16-N and signal converter model series C4000. The sensor body of each unit was modified to hold a standard DWPF $12 \mathrm{cc}$ sample vial, also known as a "peanut" vial. The purpose of this testing was to evaluate the use of this model of turbidity sensor, or meter, to provide a measurement of the sludge solids present in the SMECT based upon samples from that tank.

During discussions of the results from this study by WSE, WSL, and Savannah River National Laboratory (SRNL) personnel, an upper limit on the acceptable level of solids in SMECT samples was set at $0.14 \mathrm{wt} \%$. A "go/no-go" decision criterion was to be developed for the critical turbidity response, which is expressed in concentration units (CUs), for each Optek unit based upon the 0.14 wt $\%$ solids value. An acceptable or a "go" decision for the SMECT should reflect the situation that there is an identified risk (e.g. 5\%) for a CU response from the Optek unit to be less than the critical CU value when the solids content of the SMECT is actually $0.14 \mathrm{wt} \%$ or greater, while a "no-go" determination (i.e., an Optek $\mathrm{CU}$ response above the critical $\mathrm{CU}$ value, a conservative decision relative to risk) would lead to additional evaluations of the SMECT to better quantify the possible solids content of the tank.

A sludge simulant was used to develop standards for testing both Optek units and to determine the viability of a "go/no-go" CU response for each of the units. Statistical methods were used by SRNL to develop the critical CU value for the "go/no-go" decision for these standards for each Optek unit. Since only one sludge simulant was available for this testing, the sensitivity of these results to other simulants and to actual sludge material is not known. However, limited testing with samples from the actual DWPF process (both SRAT product samples and SMECT samples) demonstrated that the use of the "go/no-go" criteria developed from the sludge simulant testing was conservative for these samples taken from Sludge Batch $7 \mathrm{~b}$ (SB7b), the sludge batch currently being processed.

While both of the Optek units performed very reliably during this testing, there were statistically significant differences (although small on a practical scale) between the two units. Thus, testing should be conducted on any new unit of this Optek model to qualify it before it is used to support the DWPF operation. 


\section{TABLE OF CONTENTS}

LIST OF ABBREVIATIONS ................................................................................... vi

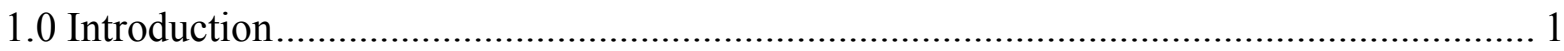

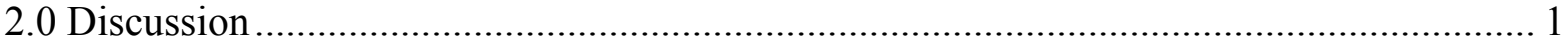

2.1 Measurements of Standards Prepared from Sludge Simulant ................................... 2

2.1.1 Results from Deionized Water Standards .......................................................... 2

2.1.2 Relating CUs to wt\% for a Sludge Simulant ...................................................... 2

2.1.3 Developing a “Go/No-Go" Criterion ............................................................ 3

2.1.4 Effect of Time-Delays before Completing Turbidity Measurements ..................... 7

2.2 Differences among Sludge Batches …................................................................ 7

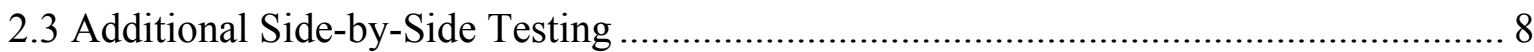

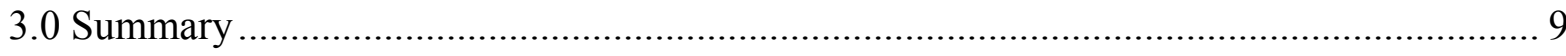

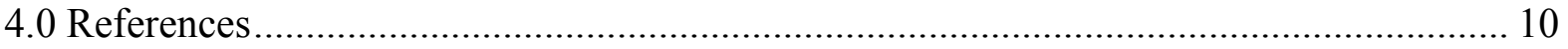




\section{LIST OF ABBREVIATIONS}

CU Concentration unit (unit of measure for turbidity meter)

DI Deionized (as in DI water)

DWPF Defense Waste Processing Facility

JMP

Pronounced "jump", commercial software

LTL

Lower tolerance limit

RCT

Recycle Collection Tank

SB7b

Sludge Batch 7b

SMECT

Slurry Mix Evaporator Condensate Tank

SRAT

Sludge Receipt and Adjustment Tank

SRNL

Savannah River National Laboratory

SRR

Savannah River Remediation, LLC

TTR

Technical Task Request

TTQAP

Task Technical and Quality Assurance Plan

WSE

Waste Solidification Engineering

WSL

Waste Services Laboratory 


\subsection{INTRODUCTION}

Savannah River Remediation's (SRR's) Defense Waste Processing Facility (DWPF) Laboratory currently tests for sludge carry-over into the Recycle Collection Tank (RCT) by evaluating the iron concentration in the Slurry Mix Evaporator Condensate Tank (SMECT) and relating this iron concentration to the amount of sludge solids present. A new method was proposed for detecting the amount of sludge in the SMECT that involves the use of a turbidity sensor. SRR's Waste Solidification Engineering (WSE) developed a test plan [1] to evaluate the use of a turbidity sensor, or meter, to provide a measurement of the sludge solids present in the SMECT based upon samples from that tank.

The calibration of the performance of the sensor was to be based on the use of sludge simulants. Different concentrations of the sludge simulant had to be prepared for laboratory testing. WSE issued a Task Technical Request [2] for the Savannah River National Laboratory (SRNL) to provide support in the selection of the concentrations of the sludge simulant to be utilized for calibrating the turbidity sensor and in evaluating the outcome of the calibration process as well as in the development of the procedural steps to complete the calibration. SRNL issued a Task Technical and Quality Assurance Plan (TTQAP) [3] in response to the SRR request, and in accordance with this plan, SRNL supported SRR in the development of the turbidity meter test plan.

The type of turbidity meter that was tested was an Optek inline control unit with a sensor model AF16-N and a signal converter model series C4000. The directions for calibration and operation of this type of meter are provided in the instruction manual for the instrument (Optek Manual - 10041004-02 - C4121-US-2012-01-26). The sensor body of the unit was modified to hold a standard DWPF 12 cubic centimeter (cc) sample vial, also known as a "peanut" vial. Two of these units were tested during this study. In this study, the two units are designated as System 1 and System 2. The serial number of System 1 is SN64217, and the serial number of System 2 is SN65164. The measurements generated by this type of turbidity meter are expressed in concentration units (CUs), which range from 0 to 6 in value.

The study activities, outlined in the test plan [1], were conducted by SRR's Waste Services Laboratory (WSL) personnel, and statistical evaluations of the measurements generated by these activities were performed by SRNL personnel. The results from these analyses were reviewed by SRNL, WSE, and WSL personnel to facilitate a decision as to the acceptable performance of the turbidity meter and as to a recommendation for its use by the DWPF Laboratory to support the measurement of the amount of sludge in the SMECT.

The purpose of this report is to document the measurements generated from this study, the results from the statistical analyses of these measurements, and the conclusions made regarding the performance of the turbidity meters evaluated by this testing. JMP Version 9.0.0 was used to support this study [4].

\subsection{DisCUSSION}

The test plan [1] outlined several activities that were to be conducted to support this investigation into the use of a turbidity meter to support the evaluation of SMECT samples for sludge solids. In the following sections, the measurements generated by these activities are presented and evaluated. 


\subsection{Measurements of Standards Prepared from Sludge Simulant}

As outlined in the test plan, WSL personnel prepared concentrations of a sludge simulant, 11-HG85869 (which was acquired from SRNL), targeting a range of weight percent (wt\%) solids concentrations. These sludge simulant "standards" were then sub-sampled by WSL to prepare a set of peanut vials that were measured by the two turbidity meters (designated as System 1 and System 2) a number of times over a period of several days. The resulting CU data were provided to SRNL for statistical analysis. Table A1 in Appendix A provides the identifiers for these peanut vial samples. The identifier (ID) designates the targeted $\mathrm{wt} \%$ solids value for the peanut vial, the bottle number (1, 2 , or 3 ) from which the peanut sample was taken and the run number $(1,2$, or 3$)$ of the peanut vial from the bottle. The turbidity meter used to conduct the measurement, the date of the measurement, the ambient temperature at the time of the measurement, and the resulting measurement itself (reported in CUs) are also provided as part of the information in Table A1.

\subsubsection{Results from Deionized Water Standards}

Included in Table A1 are the turbidity measurements of the peanut vial samples prepared using deionized (DI) water. Testing protocol item \#1 of the test plan [1] indicates that the results from these measurements are to be evaluated to provide insight into the repeatability of each of the turbidity meters and into the variation in the measurements due to differences in the peanut vials.

Exhibit A1 in Appendix A provides a plot of the CU data for these DI standards. The variation in the $\mathrm{CU}$ measurements is due to: differences in peanut vials, the position of the peanut vial (specifically, back, forward, left, or right), and day-to-day effects. It may also be noted that System 1 provides larger average CU for the standards than System 2. While the sources of these patterns in the CU data are discernible, the practical impact relative to the performance of these meters for the intended purpose under study appears to be limited. This is evident from the information in Table 1, which provides summary statistics including standard deviations for these measurements by meter including standard deviations.

Table 1. CU Data Summary Statistics for DI Standards

\begin{tabular}{|c|c|c|c|c|}
\hline Meter & Standard Wt\% & $\begin{array}{c}\text { Number of } \\
\text { Measurements }\end{array}$ & CU Average & $\begin{array}{c}\text { CU Standard } \\
\text { Deviation }\end{array}$ \\
\hline System 1 & 0 & 72 & 0.125 & 0.0354 \\
\hline System 2 & 0 & 84 & 0.106 & 0.0341 \\
\hline
\end{tabular}

\subsubsection{Relating CUs to wt\% for a Sludge Simulant}

One of the primary goals outlined in the test plan was to investigate the relationship between the CU value indicated by the turbidity measurement and the $\mathrm{wt} \%$ solids value of the standards created from a sludge simulant. The measurements provided in Table A1 allow for this investigation, and Figure 1 provides a plot of the $\mathrm{CU}$ versus $\mathrm{wt} \%$ values for each turbidity unit.

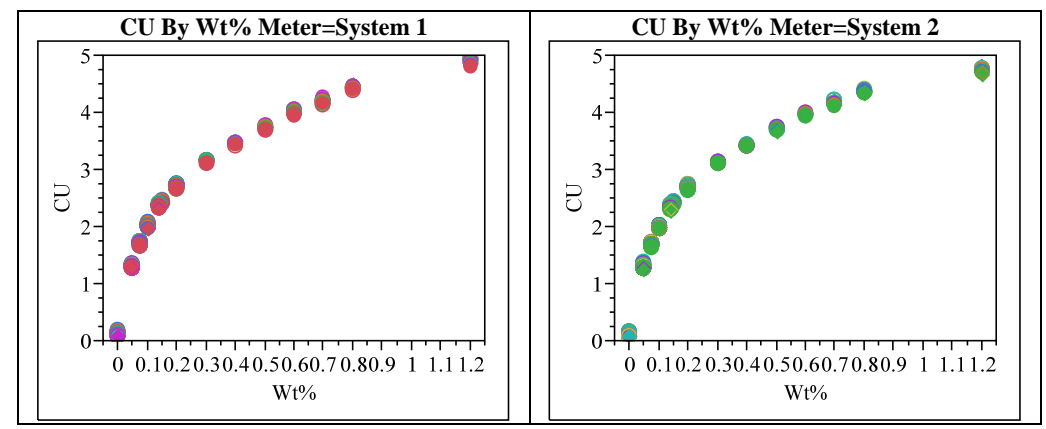

Figure 1. CU versus Simulant wt\% by Turbidity Meter 
The data of Figure 1 cover all of the measurements of Table A1, and the plots indicate very repeatable $\mathrm{CU}$ values for these sludge simulant standards for each of the two meters. However, there is value in taking a closer look at these data. Exhibit A2 in Appendix was prepared to help explore the CU values for each targeted $\mathrm{wt} \%$ solids value in more detail. In this exhibit, there is a plot of $\mathrm{CU}$ measurements for each of the turbidity meters for each of the targeted $\mathrm{wt} \%$ solids values. The measurements are grouped, as identified on the x-axis, by date and peanut vial ID. The ambient temperature for that date and the bottle and run numbers for the peanut vial are also indicated. A CU value is plotted for each of the four positions used in placing the peanut vial in the turbidity meter. The symbols used in these plots are: $\bigcirc$ - forward (FRWRD), • - backward (BACK), $\diamond$ - left (LEFT), and $\bullet$ - right (RIGHT). For each of these plots, there are several mechanisms in play that contribute to the variation seen in the $\mathrm{CU}$ values for a given wt\% solids target. Some of this variation is due to differences in the uniformity of construction among the peanut vials, (i.e., differences in the thickness of the glass walls on the four sides of each peanut vial and differences in the thickness of the glass walls between peanut vials). Given that the turbidity meter passes a beam of light through two opposing sides of a peanut vial during the measurement process, smaller differences are seen between the forward and backward pair and between the left and right pair than between other pairs of CU values for a given peanut vial. While in some instances there are day-to-day differences in the $\mathrm{CU}$ measurements of the same peanut vial, these differences do not appear to be temperature related.

\subsubsection{Developing a "Go/No-Go" Criterion}

The major objective of the test plan [1] directing this study is the evaluation of the effectiveness of this model of Optek turbidity meter to identify the presence of solids in a SMECT sample. During discussions of the results from this study by WSE, WSL, and SRNL personnel, an upper limit on the acceptable level of solids in such samples was set at $0.14 \mathrm{wt} \%$.

The question is: Can the CU measurement of a SMECT sample by an Optek unit be used to reliably determine that the solids content of the SMECT sample is no greater than $0.14 \mathrm{wt} \%$ ? The acceptable or "go" decision from this determination should be made at a high (e.g. 95\%) confidence level, while a "no-go" determination would lead to additional evaluations of the SMECT to better understand the possible solids content of the tank.

Thus, the CU measurement of a SMECT sample is to be evaluated to determine:

1. a "go" decision (i.e., an acceptable level of solids in the SMECT sample) if the $\mathrm{CU}$ value is small enough to indicate a corresponding $\mathrm{wt} \%$ value for the sample no greater than 0.14 with sufficient (e.g., $95 \%$ ) confidence. or

2. a "no-go" decision (i.e., a possibly unacceptable level of solids in the SMECT sample) if the CU value was large enough so that the "go" decision could not be made with sufficient confidence.

The measurements of the sludge simulant standards of Table A1 were used to evaluate the effectiveness of this model of Optek turbidity meter in making the determination of the solids content of samples of this material. The approach was to develop a statistical model for the relationship between the $\mathrm{wt} \%$ solids values of the samples and the corresponding CU values for each meter. Recall that these relationships are graphically indicated in Figure 1 and that the variation in the CU measurements for the peanut vials at a given $w t \%$ solids value was due to differences among peanut vials, differences in positioning the peanut vials in the instrument, and differences in day-to-day effects as discussed in the previous section.

The modeling approach was one based on how a "go/no-go" decision would be determined for each of the two Optek units on a given day utilizing only one measurement of each of the available peanut 
vial samples. The modeling approach was also focused on the relationship between the CU measurements and the corresponding wt $\%$ solids values for the interval of solids values between 0.05 $\mathrm{wt} \%$ and $0.3 \mathrm{wt} \%$. This approach was found to provide a linear model in relating the CU response to the natural logarithm of the $\mathrm{wt} \%$ solids values. Thus, the form of the model was:

$$
\text { CU value }=b_{0}+b_{1} \times \ln (w t \% \text { value })
$$

where $b_{0}$ is the term for the $y$-intercept, $b_{1}$ is the term for the slope, and $\ln (w t \%$ value) is the natural logarithm of the $\mathrm{wt} \%$ value of the standard peanut vial sample. Exhibit A3 provides the JMP results from fitting this linear model to the CU measurements by turbidity system, by date, by peanut vial position.

Some observations from Exhibit A3 are noteworthy. The results include a "Lack of Fit" evaluation for each of the linear models. A significant "Lack of Fit" for the model (at a 5\% significance level) is indicated if the "Prob > F" value in the JMP results is 0.05 or smaller. Out of the 52 fitted models from these data, only 2 yielded a statistically significant lack of fit at the $5 \%$ significance level. In addition, the estimated intercepts (the estimations of the $b_{0}$ term of the model above) and estimated slopes (the $b_{1}$ 's above) of the fitted models are very similar. Also, the $\mathrm{R}^{2}$ values for all of the fitted models are at 0.997 or above.

Thus, on any given day with only one CU measurement per peanut vial sample of the sludge simulant, there is a very reliable relationship between the wt $\%$ solids values and the CU response for both of the turbidity meters. For any given day, a statistical approach can be used to establish the "go/no-go" decision criterion as described above. This involves the determination of a lower tolerance limit (LTL) for the CU response corresponding to a wt $\%$ solids value of $0.14 \%$, and it is based upon an approach described by Miller [5]. The resulting $100(1-\alpha) \% / 100\left(1-\alpha_{0}\right) \% \mathrm{LTL}^{\mathrm{a}}$ is for $100\left(1-\alpha_{0}\right) \%$ of the CU values for these standards of the sludge simulate at a confidence of $100(1-\alpha) \%$, and the equation for the LTL for the CUs for peanut vials with a measured $\mathrm{wt}_{\mathrm{t}} \%$ of $\mathrm{w}_{\mathrm{i}}$ is given by:

$$
\mathrm{LTL}_{\mathrm{CU}}=\mathrm{b}+\mathrm{m} \cdot \ln \left(\mathrm{w}_{\mathrm{i}}\right)-\mathrm{s}\left\{\sqrt{\mathrm{pF} \mathrm{F}_{\alpha}(\mathrm{p}, \mathrm{n}-\mathrm{p})} \sqrt{\underline{\mathrm{c}}_{0}\left(\mathbf{X}^{\mathrm{T}} \mathbf{X}\right)^{-1} \underline{\mathrm{c}}_{0}{ }^{\mathrm{T}}}+\mathrm{z}_{1-\alpha_{0}} \sqrt{\frac{\mathrm{n}-\mathrm{p}}{\chi_{\alpha / 2}^{2}, \mathrm{n}-\mathrm{p}}}\right\}
$$

where

- LTL $_{\mathrm{CUi}}$ represents the lower tolerance interval (LTL) for the CU values of peanut vial samples with a wt $\%$ measurement of $\mathrm{w}_{\mathrm{i}}$,

- the values for the estimated slope $(\mathrm{m})$ and intercept (b) of the fitted model for the given day and positioning choice for the peanut vial are from the results presented in Exhibit A3,

- $\mathrm{s}$ is the root mean square error (RMSE) for the given fitted model, whose value is also available from Exhibit A3,

- $\mathrm{F}_{\alpha}(\mathrm{p}, \mathrm{n}-\mathrm{p})$ is the $100(1-\alpha) \%$ quantile of the $\mathrm{F}$ distribution, which depends on $\mathrm{n}$ (the number of data points used to fit this $p$-parameter $(\mathrm{p}=2)$ model), and the desired confidence level for bounding the estimated CU value when the $\mathrm{wt}_{\mathrm{t}} \%$ is $\mathrm{w}_{\mathrm{i}}$ is represented by $100(1-\alpha) \%$,

- the inverse product-moment matrix is represented by $\left(\mathbf{X}^{\mathrm{T}} \mathbf{X}\right)^{-1}$ where the product moment matrix contains information describing the data for the independent variable (i.e., the natural

\footnotetext{
${ }^{a}$ The LTLs were determined using the approach provided on page 124 of Miller. The notation $\mathrm{x} \% / \mathrm{y} \% \mathrm{LTL}$, such as $95 \% / 95 \%$ LTL, will be used to represent these LTLs. The notation refers to the $\mathrm{x} \%$ confidence tolerance limit with $\mathrm{y} \%$ of the CU values being greater than the LTL. The approach is based on a normal probability distribution.
} 
logarithms of the $\mathrm{wt} \%$ values) used to generate the correlation equation (the $\mathrm{wt} \%$ values of this matrix for the given linear model are given as part of the information of Table A1),

- $\underline{\mathrm{c}}_{0}$ is the vector, $\left[1 \ln \left(\mathrm{w}_{\mathrm{i}}\right)\right]$, containing the natural logarithm of the $\mathrm{wt} \%$ value, $\mathrm{w}_{\mathrm{i}}$,

- $\mathrm{z}_{1-\alpha_{0}}$ represents the one-sided $100\left(1-\alpha_{0}\right) \%$ percentile point from the standard normal distribution representing the $1-\alpha_{0}$ fraction of the model predictions to be covered, and

- $\quad \chi_{\alpha / 2, \mathrm{n}-\mathrm{p}}^{2}$ represents the lower (i.e., $100(\alpha / 2) \%$ ) percentile point of the $\chi^{2}$ distribution with $(n-p)$ degrees of freedom, used to establish an upper bound for the variance of the CU values around the fitted line.

For a given fitted model (i.e., one of the models from Exhibit A3), when equation (2) is evaluated at $95 \% / 95 \%$ for a $\mathrm{wt} \%$ value of 0.14 , the resulting LTL value provides a lower tolerance limit for $95 \%$ of the CU measurements that are likely to be generated by the given turbidity meter for samples of this sludge simulant with $\mathrm{wt} \%$ values of 0.14 or greater, and this statement is true with $95 \%$ confidence. Thus, if a peanut vial sample of the sludge simulant at an unknown $\mathrm{wt} \%$ solids concentration were to be measured by the selected turbidity meter and the resulting CU value fall below this LTL, then the conclusion is that the $\mathrm{wt} \%$ solids value of the sample is no greater than $0.14 \%$, which yields a "go" decision. A CU value at or above the LTL would lead to a "no-go" decision (i.e. the $\mathrm{wt} \%$ solids content of the sample is not at or below the $0.14 \%$ value with sufficient confidence). Table 2 provides the LTL value for each of the fitted models of Exhibit A3.

Table 2. Lower Tolerance Limits (LTLs) for CU Values for Fitted Models

\begin{tabular}{|c|c|c|c|c|}
\hline \multicolumn{2}{|c|}{ Turbidity System 1 } & & \multicolumn{2}{c|}{ Turbidity System 2 } \\
\hline Data Set & $\mathbf{9 5 / 9 5 ~ L T L}$ & & Data Set & $\mathbf{9 5}$ /95 LTL \\
\hline 14-Nov-Back & 2.301 & & 19-Nov-Back & 2.275 \\
\hline 14-Nov-Frwrd & 2.307 & & 19-Nov-Frwrd & 2.277 \\
\hline 14-Nov-Left & 2.301 & & 19-Nov-Left & 2.283 \\
\hline 14-Nov-Right & 2.3 & & 19-Nov-Right & 2.284 \\
\hline 19-Nov-Back & 2.313 & & 20-Nov-Back & 2.294 \\
\hline 19-Nov-Frwrd & 2.321 & & 20-Nov-Frwrd & 2.291 \\
\hline 19-Nov-Left & 2.314 & & 20-Nov-Left & 2.294 \\
\hline 19-Nov-Right & 2.314 & & 20-Nov-Right & 2.294 \\
\hline 20-Nov-Back & 2.324 & & 21-Nov-Back & 2.287 \\
\hline 20-Nov-Frwrd & 2.333 & & 21-Nov-Frwrd & 2.291 \\
\hline 20-Nov-Left & 2.323 & & 21-Nov-Left & 2.301 \\
\hline 20-Nov-Right & 2.327 & & 21-Nov-Right & 2.295 \\
\hline 21-Nov-Back & 2.318 & & 26-Nov-Back & 2.3 \\
\hline 21-Nov-Frwrd & 2.325 & & 26-Nov-Frwrd & 2.3 \\
\hline 21-Nov-Left & 2.32 & & 26-Nov-Left & 2.31 \\
\hline 21-Nov-Right & 2.321 & & 26-Nov-Right & 2.309 \\
\hline 26-Nov-Back & 2.323 & & 27-Nov-Back & 2.301 \\
\hline 26-Nov-Frwrd & 2.33 & & 27-Nov-Frwrd & 2.297 \\
\hline 26-Nov-Left & 2.327 & & 27-Nov-Left & 2.297 \\
\hline 26-Nov-Right & 2.33 & & 27-Nov-Right & 2.298 \\
\hline 5-Dec-Back & 2.312 & & 3-Dec-Back & 2.305 \\
\hline 5-Dec-Frwrd & 2.315 & & 3-Dec-Frwrd & 2.305 \\
\hline 5-Dec-Left & 2.315 & & 3-Dec-Left & 2.302 \\
\hline 5-Dec-Right & 2.313 & & 3-Dec-Right & 2.3 \\
\hline & & & 4-Dec-Back & 2.307 \\
\hline & & & 4-Dec-Frwrd & 2.305 \\
\hline & & & 4-Dec-Left & 2.309 \\
\hline & & & & 2.305 \\
\hline
\end{tabular}


Figure 2 provides comparisons of these two sets of LTL values. The figure includes Levene's test for a difference in variation for the two sets of values. The p-value for this test is 0.8575 , which indicates that the claim that the variances of the two sets are equal cannot be rejected at a $5 \%$ significance level. The figure also contains a statistical comparison of the means of the two sets of LTL values, which does indicate that the means for the two turbidity meters are different at a significant level of 5\% (note the $p$-value indicated in Figure 2 by Prob $>|t|$ is $<0.0001$ ). Thus, even though the variation in these LTL values is comparable for the two meters, the difference between their mean LTL values (2.318 CU for System 1 and 2.297 CU for System 2) is statistically significant.

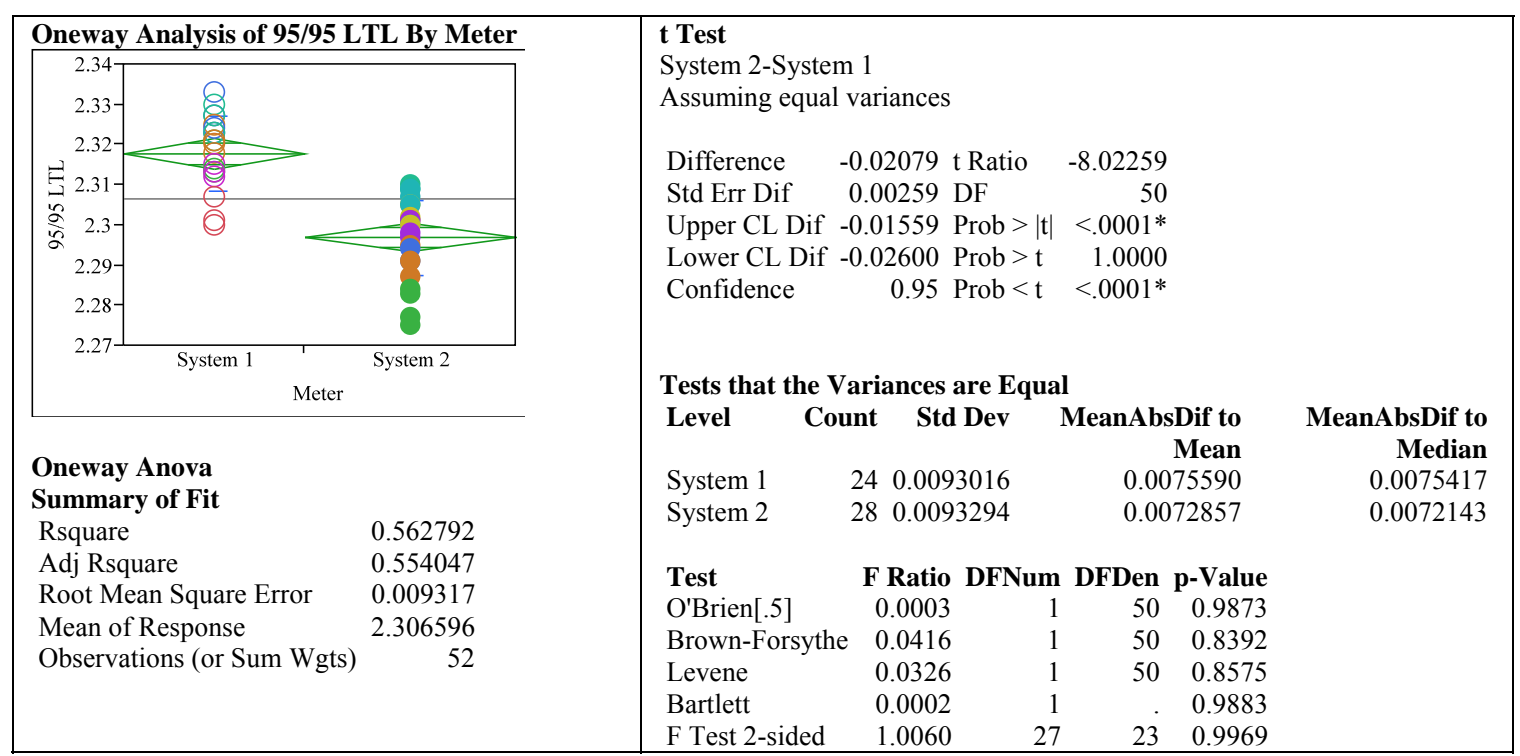

Figure 2. Comparison of LTL Values for the Two Turbidity Meters

Note than the $\%$ relative standard deviations in these sets of LTL are $100 \% \times 0.0093016 / 2.318=$ $0.4 \%$ for System 1 and $100 \% \times 0.0093294 / 2.297=0.4 \%$ for System 2 . Thus, there is little variation in the LTL results for a particular meter, but there is a statistically significant difference between the two meters.

Exhibit A4 provides the results of an additional investigation into these LTL values. This exhibit, which was generated using JMP [4], provides descriptive statistics as well as a test of the hypothesis of a normal distribution for each set of LTL values. The p-values for this test (indicated by the Prob $<\mathrm{W}$ values) are 0.3101 for System 1 and 0.0949 for System 2, and as a result the normal hypothesis for the LTL values cannot be rejected for either turbidity system. Using the normal distribution as a basis, JMP was used to determine a one-sided, lower tolerance interval for each set of LTL values. These values are provided in Exhibit A4, and they are 2.296 CU for turbidity System 1 and 2.276 CU for turbidity System 2. Thus, these are the critical values that would be used for these two turbidity meters to establish a "go/no-go" decision regarding a wt $\%$ solids value of $0.14 \mathrm{wt} \%$ in measuring peanut samples of the simulated sludge used in this study with the wt\% solids of these samples being unknown.

The "go/no-go" decision criterion for the critical turbidity response for System 2 (the installed unit) based upon the $0.14 \mathrm{wt} \%$ solids value may be restated as follows. An acceptable or a "go" decision for the SMECT should reflect the situation that there is an identified risk (e.g. 5\%) for a CU response from this Optek unit to be less than the critical $\mathrm{CU}$ value $(2.276 \mathrm{CU})$ when the solids content of the 
SMECT is actually $0.14 \mathrm{wt} \%$ or greater, while a "no-go" determination (i.e., an Optek CU response > $2.276 \mathrm{CU}$ ) a conservative decision relative to risk) would lead to additional evaluations of the SMECT to better understand the possible solids content of the tank.

\subsubsection{Effect of Time-Delays before Completing Turbidity Measurements}

While the CU measurements discussed so far in this report were completed without any delays between the time the peanut vial sample was shaken and well-mixed, the time the vial was placed in the turbidity meter, and the time a CU value determined, the test plan [1] requested that settling time effects be investigated as part of this study. Table 2 provides the results from this investigation.

Table 2. CU Measurements over Time

\begin{tabular}{|c|c|c|c|c|c|c|}
\hline Time (sec) & $0.05 \mathrm{Wt} \%$ & $0.1 \mathrm{Wt} \%$ & $0.15 \mathrm{Wt} \%$ & $0.2 \mathrm{Wt} \%$ & $0.4 \mathrm{Wt} \%$ & $0.8 \mathrm{Wt} \%$ \\
\hline 10 & 1.32 & 2.01 & 2.47 & 2.76 & \begin{tabular}{|l|}
3.48 \\
\end{tabular} & \begin{tabular}{|l|}
4.47 \\
\end{tabular} \\
\hline 20 & 1.33 & 2 & 2.46 & 2.75 & 3.47 & 4.45 \\
\hline 30 & 1.32 & 2 & 2.46 & 2.75 & 3.46 & 4.44 \\
\hline 40 & 1.32 & 1.99 & 2.45 & 2.74 & 3.45 & 4.42 \\
\hline 50 & 1.32 & 1.99 & 2.45 & 2.74 & 3.44 & 4.38 \\
\hline 60 & 1.32 & 1.99 & 2.44 & 2.73 & 3.43 & 4.33 \\
\hline 70 & 1.32 & 1.99 & 2.44 & 2.73 & 3.42 & 4.25 \\
\hline 80 & 1.32 & 1.98 & 2.44 & 2.73 & 3.41 & 4.14 \\
\hline 90 & 1.32 & 1.98 & 2.44 & 2.73 & 3.4 & 4 \\
\hline 100 & 1.31 & 1.98 & 2.43 & 2.72 & 3.39 & 3.84 \\
\hline 110 & 1.31 & 1.98 & 2.43 & 2.72 & 3.38 & 3.66 \\
\hline 120 & 1.31 & 1.98 & 2.43 & 2.72 & 3.37 & 3.48 \\
\hline 130 & 1.31 & 1.98 & 2.43 & 2.72 & 3.35 & 3.28 \\
\hline 140 & 1.31 & 1.97 & 2.42 & 2.72 & 3.34 & 3.11 \\
\hline 150 & 1.31 & 1.97 & 2.42 & 2.71 & 3.32 & 2.94 \\
\hline 160 & 1.31 & 1.97 & 2.42 & 2.71 & 3.3 & 2.82 \\
\hline 170 & 1.31 & 1.97 & 2.42 & 2.71 & 3.29 & 2.73 \\
\hline 180 & 1.31 & 1.97 & 2.42 & 2.71 & 3.26 & 2.65 \\
\hline 190 & 1.31 & 1.97 & 2.41 & 2.7 & 3.23 & 2.59 \\
\hline 200 & 1.31 & 1.96 & 2.41 & 2.7 & 3.2 & 2.52 \\
\hline 210 & 1.31 & 1.96 & 2.41 & 2.69 & 3.16 & 2.46 \\
\hline 220 & 1.31 & 1.96 & 2.41 & 2.69 & 3.13 & 2.36 \\
\hline 230 & 1.3 & 1.96 & 2.41 & 2.69 & 3.09 & 2.31 \\
\hline 240 & 1.3 & 1.96 & 2.4 & 2.69 & 3.06 & 2.26 \\
\hline 250 & 1.3 & 1.96 & 2.4 & 2.69 & 3.03 & 2.23 \\
\hline 260 & 1.3 & 1.96 & 2.4 & 2.68 & 3 & 2.21 \\
\hline 270 & 1.3 & 1.95 & 2.4 & 2.68 & 2.97 & 2.17 \\
\hline 280 & 1.3 & 1.95 & 2.4 & 2.68 & 2.93 & 2.15 \\
\hline 290 & 1.3 & 1.95 & 2.39 & 2.68 & 2.89 & 2.11 \\
\hline 300 & 1.3 & 1.95 & 2.39 & 2.67 & 2.86 & 2.07 \\
\hline
\end{tabular}

Exhibit A5 in Appendix A provides a plot these CU responses over time for each of the standards of the simulated sludge used in this study. A review of these plots suggests that sample handling is an issue and that the procedures providing guidance for the completion of these measurements for SMECT samples must ensure that the sample is well-mixed as the peanut vial is introduced into the turbidity meter. Also, note that the results shown here are only for the simulated sludge used in this study and that samples of real sludge material may be more or less sensitive to these timing issues.

\subsection{Differences among Sludge Batches}

Another goal of the test plan [1] was to use a second sludge simulant as part of this study. However, a second simulant was not available, but two sets of standards were developed using a sample of the product material from the Sludge Receipt and Adjustment Tank (SRAT). Table 3 provides the CU measurements for each set of SRAT standards, which were made and measured using the installed 
turbidity meter (i.e., System 2) on two different days. Measurements of standards at the same wt\% values for the sludge simulant are also provided in this table.

Table 3. CU Measurements for SRAT Standards

\begin{tabular}{|c|c|c|c|}
\hline Day & Standard $\mathrm{wt} \%$ & SRAT Sample (CU) & Simulant Sample (CU) \\
\hline 1 & 0.05 & 1.30 & 1.31 \\
\hline 1 & 0.10 & 2.24 & 2.02 \\
\hline 1 & 0.14 & 2.65 & 2.35 \\
\hline 2 & 0.05 & 1.29 & 1.31 \\
\hline 2 & 0.10 & 2.26 & 2.02 \\
\hline 2 & 0.14 & 2.77 & 2.35 \\
\hline
\end{tabular}

Note that while there is good repeatability in these results over both days, the CU measurements for the SRAT material are larger than the CU measurements for their simulant counterparts. Thus, these side-by-side comparisons suggest that any "go/no-go" criteria developed using the CU measurements for the sludge simulant standards may be conservative (i.e., lead to more "no-go" outcomes when the true $\mathrm{wt} \%$ is actually less than $0.14 \mathrm{wt} \%$ ) if applied to the CU measurements for the sludge represented by this SRAT product material.

\subsection{Additional Side-by-Side Testing}

The test plan guiding this study included a series of "side-by-side" testing that was to involve the measurement of standards developed from SRAT samples during the processing of the current sludge batch, Sludge Batch 7b (SB7b). While the results from Section 2.2 provide some feedback on such "side-by-side" (actual versus simulated sludge) comparisons of actual versus simulated sludge, additional "side-by-side" testing involving actual SMECT samples was conducted by WSL personnel as part of this study. The turbidity measurement results from this testing are provided in Table 4.

Table 4. CU Measurements of SMECT Samples

\begin{tabular}{|c|c|c|c|c|c|c|c|c|c|c|}
\hline DATE & 7-Dec-12 & 9-Dec-12 & $\begin{array}{c}\text { 11-Dec- } \\
12\end{array}$ & $\begin{array}{c}\text { 13-Dec- } \\
12\end{array}$ & $\begin{array}{c}\text { 17-Dec- } \\
12 \\
\end{array}$ & $\begin{array}{c}\text { 19-Dec- } \\
12\end{array}$ & $\begin{array}{c}\text { 20-Dec- } \\
12\end{array}$ & $\begin{array}{c}\text { 22-Dec- } \\
12\end{array}$ & $\begin{array}{c}\text { 26-Dec- } \\
12 \\
\end{array}$ & $\begin{array}{c}\text { 27-Dec- } \\
12\end{array}$ \\
\hline LIMS ID & 200015581 & 200015590 & 200015601 & 200015614 & 200015655 & 200015662 & 200015686 & 200015697 & 200015716 & 200015721 \\
\hline Batch & 4060 & 4061 & 4062 & 4063 & 4065 & 4066 & 4067 & 4067 & 4068 & 4069 \\
\hline $\mathrm{Fe}$ MA corr $1 \mathrm{mg} / \mathrm{L}$ & 381 & 188 & $<121$ & $<119$ & $<120$ & $<121$ & $<127$ & $<123$ & $<124$ & 941 \\
\hline Fe_MA corr $2 \mathrm{mg} / \mathrm{L}$ & 298 & 191 & 128 & $<118$ & $<122$ & $<114$ & $<127$ & $<115$ & $<118$ & 956 \\
\hline Average $\mathrm{mg} / \mathrm{L}$ & 339 & 190 & $<128$ & $<119$ & $<122$ & $<121$ & $<127$ & $<123$ & $<124$ & 949 \\
\hline \multicolumn{11}{|l|}{ Turbidity CU } \\
\hline rep 1 & 3.76 & 2.94 & 2.27 & 1.76 & 0.58 & 1.15 & 1.51 & 1.51 & 1.11 & 4.74 \\
\hline rep 2 & 3.78 & 2.92 & 2.28 & ------ & 0.63 & 1.08 & 1.54 & 1.54 & 1.11 & 3.78 \\
\hline Average CU & 3.77 & 2.93 & 2.28 & 1.76 & 0.61 & 1.12 & 1.53 & 1.53 & 1.11 & 4.26 \\
\hline $\begin{array}{c}\text { Standard Open } \\
{[0.14 \mathrm{wt} \%]}\end{array}$ & 2.34 & 2.34 & 2.33 & 2.34 & 2.32 & 2.36 & 2.36 & 2.34 & 2.36 & 2.34 \\
\hline $\begin{array}{c}\text { Standard Close } \\
{[0.14 \mathrm{wt} \%]}\end{array}$ & 2.34 & 2.34 & 2.35 & 2.34 & 2.33 & 2.36 & 2.36 & 2.37 & 2.35 & 2.35 \\
\hline
\end{tabular}

Two SMECT samples were measured using the installed meter on each of the days indicated in this table. Measurements of a $0.14 \mathrm{wt} \%$ standard prepared from the sludge simulant used for this study which were made at the beginning and the end of each measurement set are also provided in this table. These measurements were made using the installed turbidity meter, i.e., System 2. The laboratory information management system (LIMS) identifier (ID) and batch number for the results are also provided in the table along with the iron $(\mathrm{Fe})$ elemental measurement from each mixed acid (MA) prepared SMECT sample. The Fe numbers are the critical points of comparison for this testing. 
Recall that when the "go/no-go" decision yields a "no-go" outcome, additional testing in the form of Fe measurements is to be conducted for the SMECT samples. Using the 2.276 CU value developed above as the "go/no-go" decision point for System 2 (for the sludge simulant), the data shaded in green in Table 4 would have been "go" decisions while the data shaded in red would have been "nogo" situations, which would have required Fe measurements for the corresponding SMECT samples. Also note that for all of the "go" situations (i.e., those in green), the corresponding Fe measurements are below detection, which implies minimal sludge content (i.e., an acceptable amount of solids content) for these situations. A closer look at the Fe results from Table 4 indicates a sludge carry-over into the SMECT on 7-Dec with the sludge solids in the SMECT being diluted during subsequent processing until a second sludge carry-over on 27-Dec. Significantly, the System 2 turbidity measurements for these SMECT samples reliably mimic the pattern revealed by the Fe measurements, with the "go/no-go" decision point (i.e., the $2.276 \mathrm{CU}$ value) conservatively protecting the operation from an incorrect "go" decision.

\subsection{SUMMARY}

The DWPF Laboratory currently tests for sludge carry-over into the RCT by evaluating the iron concentration in the SMECT and relating this iron concentration to the amount of sludge solids present. A new method was proposed for detecting the amount of sludge in the SMECT that involves the use of an Optek turbidity sensor. WSL personnel conducted testing on two of these units following a test plan developed by WSE. Both Optek units (SN64217 and SN65164) use sensor model AF16-N and signal converter model series C4000. The sensor body of each unit was modified to hold a standard DWPF 12 cc sample vial, also known as a "peanut" vial. The purpose of this testing was to evaluate the use of this model of turbidity sensor, or meter, to provide a measurement of the sludge solids present in the SMECT based upon samples from that tank.

During discussions of the results from this study by WSE, WSL, and SRNL personnel, an upper limit on the acceptable level of solids in SMECT samples was set at $0.14 \mathrm{wt} \%$. A "go/no-go" decision criterion was developed for the critical turbidity response, which is expressed in concentrations units (CUs), for each Optek unit based upon the $0.14 \mathrm{wt} \%$ solids value. An acceptable or "go" decision for the SMECT should reflect the situation that there is an identified risk (e.g. 5\%) for a CU response from the Optek unit less than the critical CU value when the solids content of the SMECT is actually $0.14 \mathrm{wt} \%$ or greater, while a "no-go" determination (i.e., an Optek CU response above the critical CU value, a conservative decision relative to risk) would lead to additional evaluations of the SMECT to better quantify the possible solids content of the tank.

A sludge simulant was used to develop standards for testing both Optek units and to determine the viability of a "go/no-go" CU response for each of the units. Statistical methods were used by SRNL to develop the critical CU value for the "go/no-go" decision for these standards for each Optek unit. Since only one sludge simulant was available for this testing, the sensitivity of these results to other simulants and to actual sludge material is not known. However, limited testing with samples from the actual DWPF process (both SRAT product samples and SMECT samples) demonstrated that the use of the "go/no-go" criteria developed from the sludge simulant testing was conservative for these samples taken from the sludge batch currently being processed, SB7b.

Also, while both of the two Optek units performed very reliably during this testing, there were statistically significant differences (although small on a practical scale) between the two units. Thus, testing should be conducted on any new unit of this Optek model to qualify its performance before it is used to support the DWPF operation. 


\subsection{REFERENCES}

[1] Behrouzi, A.M., “Test Plan: Turbidity Meter Testing,” SRR-WSE-2012-00208, November $16,2012$.

[2] Behrouzi, A.M., "Technical Task Request: Inputs for Turbidity Meter Testing and Data Analysis,” HLW-DWPF-TTR-2013-0007, November 8, 2012.

[3] Edwards, T.B., "Task Technical and Quality Assurance Plan: Inputs for Turbidity Meter Testing and Data Analysis," SRNL-RP-2012-00821, November 14, 2012.

[4] JMP ${ }^{\circledR}$ Version 9.0.0, SAS Institute Inc., Cary, NC, 2010.

[5] Miller, R.G., Simultaneous Statistical Inference, Second Edition, Springer-Verlag, New York, 1989. 
APPENDIX A. SupPlemental TABLES AND EXHIBITS

Table A1. Concentration Unit (CU) Measurements

\begin{tabular}{|c|c|c|c|c|c|c|c|c|c|c|}
\hline \multirow{2}{*}{ Meter } & \multirow{2}{*}{$\begin{array}{l}\text { Peanut } \\
\text { Vial ID }\end{array}$} & \multirow{2}{*}{ Wt $\%$} & \multirow{2}{*}{ Bottle } & \multirow{2}{*}{ Run } & \multirow{2}{*}{ Date } & \multirow{2}{*}{ Temp } & \multicolumn{4}{|c|}{ Concentration Units (CUs) } \\
\hline & & & & & & & Back & Forward & Left & Right \\
\hline System 1 & DI 1-1 & 0 & 1 & 1 & 14-Nov & 73 & 0.09 & 0.09 & 0.1 & 0.1 \\
\hline System 1 & DI 1-1 & 0 & 1 & 1 & 19-Nov & 73 & 0.09 & 0.09 & 0.11 & 0.12 \\
\hline System 1 & DI $1-1$ & 0 & 1 & 1 & 20-Nov & 63 & 0.09 & 0.1 & 0.11 & 0.12 \\
\hline System 1 & DI 1-1 & 0 & 1 & 1 & 21-Nov & 70 & 0.09 & 0.08 & 0.11 & 0.11 \\
\hline System 1 & DI $1-1$ & 0 & 1 & 1 & 26-Nov & 70 & 0.08 & 0.08 & 0.11 & 0.12 \\
\hline System 1 & DI 1-1 & 0 & 1 & 1 & 5-Dec & 74 & 0.09 & 0.08 & 0.08 & 0.09 \\
\hline System 1 & DI 1-2 & 0 & 1 & 2 & 14-Nov & 73 & 0.14 & 0.17 & 0.16 & 0.16 \\
\hline System 1 & DI $1-2$ & 0 & 1 & 2 & 19-Nov & 73 & 0.13 & 0.15 & 0.1 & 0.08 \\
\hline System 1 & DI 1-2 & 0 & 1 & 2 & 20-Nov & 63 & 0.12 & 0.15 & 0.1 & 0.08 \\
\hline System 1 & DI $1-2$ & 0 & 1 & 2 & 21-Nov & 70 & 0.12 & 0.14 & 0.09 & 0.08 \\
\hline System 1 & DI 1-2 & 0 & 1 & 2 & 26-Nov & 70 & 0.12 & 0.13 & 0.09 & 0.08 \\
\hline System 1 & DI 1-2 & 0 & 1 & 2 & 5-Dec & 74 & 0.11 & 0.13 & 0.08 & 0.07 \\
\hline System 1 & DI 1-3 & 0 & 1 & 3 & 14-Nov & 73 & 0.13 & 0.17 & 0.16 & 0.17 \\
\hline System 1 & DI 1-3 & 0 & 1 & 3 & 19-Nov & 73 & 0.13 & 0.18 & 0.17 & 0.18 \\
\hline System 1 & DI $1-3$ & 0 & 1 & 3 & 20-Nov & 63 & 0.14 & 0.19 & 0.16 & 0.18 \\
\hline System 1 & DI 1-3 & 0 & 1 & 3 & 21-Nov & 70 & 0.13 & 0.17 & 0.16 & 0.18 \\
\hline System 1 & DI $1-3$ & 0 & 1 & 3 & 26-Nov & 70 & 0.13 & 0.18 & 0.17 & 0.18 \\
\hline System 1 & DI $1-3$ & 0 & 1 & 3 & 5-Dec & 74 & 0.14 & 0.18 & 0.15 & 0.17 \\
\hline System 1 & $0.051-1$ & 0.05 & 1 & 1 & 14-Nov & 73 & 1.3 & 1.3 & 1.28 & 1.27 \\
\hline System 1 & $0.051-1$ & 0.05 & 1 & 1 & 19-Nov & 73 & 1.31 & 1.31 & 1.29 & 1.3 \\
\hline System 1 & $0.051-1$ & 0.05 & 1 & 1 & 20-Nov & 63 & 1.32 & 1.33 & 1.3 & 1.3 \\
\hline System 1 & $0.051-1$ & 0.05 & 1 & 1 & 21-Nov & 70 & 1.32 & 1.33 & 1.3 & 1.3 \\
\hline System 1 & $0.051-1$ & 0.05 & 1 & 1 & 26-Nov & 70 & 1.33 & 1.34 & 1.3 & 1.3 \\
\hline System 1 & $0.051-1$ & 0.05 & 1 & 1 & 5-Dec & 74 & 1.31 & 1.32 & 1.31 & 1.29 \\
\hline System 1 & $0.051-2$ & 0.05 & 1 & 2 & 14-Nov & 73 & 1.28 & 1.28 & 1.29 & 1.28 \\
\hline System 1 & $0.051-2$ & 0.05 & 1 & 2 & 19-Nov & 73 & 1.28 & 1.29 & 1.3 & 1.29 \\
\hline System 1 & $0.051-2$ & 0.05 & 1 & 2 & 20-Nov & 63 & 1.3 & 1.31 & 1.33 & 1.31 \\
\hline System 1 & $0.051-2$ & 0.05 & 1 & 2 & 21-Nov & 70 & 1.29 & 1.3 & 1.31 & 1.31 \\
\hline System 1 & $0.051-2$ & 0.05 & 1 & 2 & 26-Nov & 70 & 1.3 & 1.31 & 1.32 & 1.32 \\
\hline System 1 & $0.051-2$ & 0.05 & 1 & 2 & 5-Dec & 74 & 1.31 & 1.31 & 1.32 & 1.32 \\
\hline System 1 & $0.051-3$ & 0.05 & 1 & 3 & 14-Nov & 73 & 1.28 & 1.28 & 1.29 & 1.28 \\
\hline System 1 & $0.051-3$ & 0.05 & 1 & 3 & 19-Nov & 73 & 1.31 & 1.32 & 1.3 & 1.29 \\
\hline System 1 & $0.051-3$ & 0.05 & 1 & 3 & 20-Nov & 63 & 1.32 & 1.33 & 1.31 & 1.32 \\
\hline System 1 & $0.051-3$ & 0.05 & 1 & 3 & 21-Nov & 70 & 1.32 & 1.33 & 1.32 & 1.33 \\
\hline System 1 & $0.051-3$ & 0.05 & 1 & 3 & 26-Nov & 70 & 1.31 & 1.33 & 1.33 & 1.33 \\
\hline System 1 & $0.051-3$ & 0.05 & 1 & 3 & 5-Dec & 74 & 1.31 & 1.32 & 1.3 & 1.3 \\
\hline System 1 & $0.052-1$ & 0.05 & 2 & 1 & 14-Nov & 73 & 1.29 & 1.28 & 1.29 & 1.31 \\
\hline System 1 & $0.052-1$ & 0.05 & 2 & 1 & 19-Nov & 73 & 1.3 & 1.31 & 1.31 & 1.3 \\
\hline System 1 & $0.052-1$ & 0.05 & 2 & 1 & 20-Nov & 63 & 1.31 & 1.31 & 1.31 & 1.32 \\
\hline System 1 & $0.052-1$ & 0.05 & 2 & 1 & 21-Nov & 70 & 1.31 & 1.31 & 1.32 & 1.31 \\
\hline System 1 & $0.052-1$ & 0.05 & 2 & 1 & 26-Nov & 70 & 1.32 & 1.32 & 1.33 & 1.33 \\
\hline System 1 & $0.052-1$ & 0.05 & 2 & 1 & $5-\mathrm{Dec}$ & 74 & 1.3 & 1.29 & 1.3 & 1.31 \\
\hline System 1 & $0.052-2$ & 0.05 & 2 & 2 & 14-Nov & 73 & 1.3 & 1.31 & 1.31 & 1.3 \\
\hline System 1 & $0.052-2$ & 0.05 & 2 & 2 & 19-Nov & 73 & 1.31 & 1.31 & 1.34 & 1.33 \\
\hline System 1 & $0.052-2$ & 0.05 & 2 & 2 & 20-Nov & 63 & 1.32 & 1.32 & 1.36 & 1.34 \\
\hline System 1 & $0.052-2$ & 0.05 & 2 & 2 & 21-Nov & 70 & 1.33 & 1.31 & 1.35 & 1.34 \\
\hline System 1 & $0.052-2$ & 0.05 & 2 & 2 & 26-Nov & 70 & 1.33 & 1.3 & 1.37 & 1.35 \\
\hline System 1 & $0.052-2$ & 0.05 & 2 & 2 & 5-Dec & 74 & 1.36 & 1.36 & 1.34 & 1.33 \\
\hline System 1 & $0.052-3$ & 0.05 & 2 & 3 & 14-Nov & 73 & 1.3 & 1.28 & 1.29 & 1.28 \\
\hline System 1 & $0.052-3$ & 0.05 & 2 & 3 & 19-Nov & 73 & 1.32 & 1.32 & 1.3 & 1.31 \\
\hline System 1 & $0.052-3$ & 0.05 & 2 & 3 & 20-Nov & 63 & 1.33 & 1.33 & 1.32 & 1.32 \\
\hline System 1 & $0.052-3$ & 0.05 & 2 & 3 & 21-Nov & 70 & 1.31 & 1.33 & 1.32 & 1.31 \\
\hline System 1 & $0.052-3$ & 0.05 & 2 & 3 & 26-Nov & 70 & 1.33 & 1.34 & 1.32 & 1.32 \\
\hline System 1 & $0.052-3$ & 0.05 & 2 & 3 & 5-Dec & 74 & 1.31 & 1.31 & 1.3 & 1.29 \\
\hline System 1 & $0.053-1$ & 0.05 & 3 & 1 & 14-Nov & 73 & 1.28 & 1.29 & 1.29 & 1.29 \\
\hline System 1 & $0.053-1$ & 0.05 & 3 & 1 & 19-Nov & 73 & 1.3 & 1.31 & 1.31 & 1.31 \\
\hline System 1 & $0.053-1$ & 0.05 & 3 & 1 & 20-Nov & 63 & 1.31 & 1.33 & 1.32 & 1.32 \\
\hline System 1 & $0.053-1$ & 0.05 & 3 & 1 & 21-Nov & 70 & 1.3 & 1.32 & 1.31 & 1.32 \\
\hline System 1 & $0.053-1$ & 0.05 & 3 & 1 & 26-Nov & 70 & 1.31 & 1.32 & 1.32 & 1.33 \\
\hline System 1 & $0.053-1$ & 0.05 & 3 & 1 & 5-Dec & 74 & 1.29 & 1.29 & 1.3 & 1.3 \\
\hline System 1 & $0.053-2$ & 0.05 & 3 & 2 & 14-Nov & 73 & 1.29 & 1.3 & 1.3 & 1.29 \\
\hline System 1 & $0.053-2$ & 0.05 & 3 & 2 & 19-Nov & 73 & 1.32 & 1.32 & 1.33 & 1.31 \\
\hline System 1 & $0.053-2$ & 0.05 & 3 & 2 & 20-Nov & 63 & 1.33 & 1.33 & 1.34 & 1.33 \\
\hline System 1 & $0.053-2$ & 0.05 & 3 & 2 & 21-Nov & 70 & 1.32 & 1.33 & 1.33 & 1.32 \\
\hline System 1 & $0.053-2$ & 0.05 & 3 & 2 & 26-Nov & 70 & 1.34 & 1.34 & 1.34 & 1.33 \\
\hline System 1 & $0.053-2$ & 0.05 & 3 & 2 & 5-Dec & 74 & 1.33 & 1.32 & 1.33 & 1.31 \\
\hline System 1 & $0.053-3$ & 0.05 & 3 & 3 & 14-Nov & 73 & 1.28 & 1.29 & 1.28 & 1.27 \\
\hline
\end{tabular}


Table A1. Concentration Unit (CU) Measurements

\begin{tabular}{|c|c|c|c|c|c|c|c|c|c|c|}
\hline \multirow{2}{*}{ Meter } & \multirow{2}{*}{$\begin{array}{l}\text { Peanut } \\
\text { Vial ID } \\
\end{array}$} & \multirow{2}{*}{ Wt\% } & \multirow{2}{*}{ Bottle } & \multirow{2}{*}{ Run } & \multirow{2}{*}{ Date } & \multirow{2}{*}{ Temp } & & ncentratio & Jnits ( & \\
\hline & & & & & & & Back & Forward & Left & Right \\
\hline System 1 & $0.053-3$ & 0.05 & 3 & 3 & 19-Nov & 73 & 1.31 & 1.31 & 1.3 & 1.29 \\
\hline System 1 & $0.053-3$ & 0.05 & 3 & 3 & 20-Nov & 63 & 1.32 & 1.32 & 1.31 & 1.3 \\
\hline System 1 & $0.053-3$ & 0.05 & 3 & 3 & 21-Nov & 70 & 1.31 & 1.31 & 1.31 & 1.29 \\
\hline System 1 & $0.053-3$ & 0.05 & 3 & 3 & 26-Nov & 70 & 1.32 & 1.32 & 1.32 & 1.3 \\
\hline System 1 & $0.053-3$ & 0.05 & 3 & 3 & 5-Dec & 74 & 1.3 & 1.3 & 1.28 & 1.28 \\
\hline System 1 & $0.0751-1$ & 0.075 & 1 & 1 & 14-Nov & 73 & 1.7 & 1.71 & 1.71 & 1.7 \\
\hline System 1 & 0.075 1-1 & 0.075 & 1 & 1 & 19-Nov & 73 & 1.73 & 1.73 & 1.72 & 1.73 \\
\hline System 1 & $0.0751-1$ & 0.075 & 1 & 1 & 20-Nov & 63 & 1.73 & 1.73 & 1.74 & 1.74 \\
\hline System 1 & $0.0751-1$ & 0.075 & 1 & 1 & 21-Nov & 70 & 1.73 & 1.74 & 1.74 & 1.74 \\
\hline System 1 & $0.0751-1$ & 0.075 & 1 & 1 & 26-Nov & 70 & 1.75 & 1.74 & 1.74 & 1.75 \\
\hline System 1 & $0.0751-1$ & 0.075 & 1 & 1 & 5-Dec & 74 & 1.71 & 1.7 & 1.72 & 1.71 \\
\hline System 1 & $0.0751-2$ & 0.075 & 1 & 2 & 14-Nov & 73 & 1.66 & 1.67 & 1.66 & 1.66 \\
\hline System 1 & $0.075 \quad 1-2$ & 0.075 & 1 & 2 & 19-Nov & 73 & 1.67 & 1.68 & 1.67 & 1.67 \\
\hline System 1 & $0.0751-2$ & 0.075 & 1 & 2 & 20-Nov & 63 & 1.69 & 1.69 & 1.68 & 1.69 \\
\hline System 1 & $0.0751-2$ & 0.075 & 1 & 2 & 21-Nov & 70 & 1.67 & 1.68 & 1.67 & 1.68 \\
\hline System 1 & $0.0751-2$ & 0.075 & 1 & 2 & 26-Nov & 70 & 1.67 & 1.68 & 1.68 & 1.69 \\
\hline System 1 & $0.0751-2$ & 0.075 & 1 & 2 & 5-Dec & 74 & 1.67 & 1.68 & 1.68 & 1.67 \\
\hline System 1 & $0.0751-3$ & 0.075 & 1 & 3 & 14-Nov & 73 & 1.69 & 1.71 & 1.7 & 1.7 \\
\hline System 1 & $0.0751-3$ & 0.075 & 1 & 3 & 19-Nov & 73 & 1.72 & 1.72 & 1.71 & 1.71 \\
\hline System 1 & $0.075 \quad 1-3$ & 0.075 & 1 & 3 & 20-Nov & 63 & 1.73 & 1.73 & 1.72 & 1.71 \\
\hline System 1 & $0.0751-3$ & 0.075 & 1 & 3 & 21-Nov & 70 & 1.72 & 1.73 & 1.73 & 1.71 \\
\hline System 1 & $0.0751-3$ & 0.075 & 1 & 3 & 26-Nov & 70 & 1.73 & 1.74 & 1.74 & 1.72 \\
\hline System 1 & $0.0751-3$ & 0.075 & 1 & 3 & 5-Dec & 74 & 1.72 & 1.73 & 1.72 & 1.7 \\
\hline System 1 & $0.0752-1$ & 0.075 & 2 & 1 & 14-Nov & 73 & 1.72 & 1.7 & 1.7 & 1.7 \\
\hline System 1 & $0.0752-1$ & 0.075 & 2 & 1 & 19-Nov & 73 & 1.73 & 1.71 & 1.7 & 1.72 \\
\hline System 1 & $0.0752-1$ & 0.075 & 2 & 1 & 20-Nov & 63 & 1.73 & 1.73 & 1.72 & 1.73 \\
\hline System 1 & $0.0752-1$ & 0.075 & 2 & 1 & 21-Nov & 70 & 1.74 & 1.72 & 1.71 & 1.72 \\
\hline System 1 & $0.0752-1$ & 0.075 & 2 & 1 & 26-Nov & 70 & 1.75 & 1.73 & 1.73 & 1.74 \\
\hline System 1 & $0.0752-1$ & 0.075 & 2 & 1 & 5-Dec & 74 & 1.72 & 1.7 & 1.69 & 1.71 \\
\hline System 1 & $0.0752-2$ & 0.075 & 2 & 2 & 14-Nov & 73 & 1.72 & 1.73 & 1.71 & 1.71 \\
\hline System 1 & $0.0752-2$ & 0.075 & 2 & 2 & 19-Nov & 73 & 1.74 & 1.74 & 1.72 & 1.71 \\
\hline System 1 & $0.0752-2$ & 0.075 & 2 & 2 & 20-Nov & 63 & 1.75 & 1.75 & 1.73 & 1.72 \\
\hline System 1 & $0.0752-2$ & 0.075 & 2 & 2 & 21-Nov & 70 & 1.75 & 1.74 & 1.73 & 1.72 \\
\hline System 1 & $0.0752-2$ & 0.075 & 2 & 2 & 26-Nov & 70 & 1.76 & 1.76 & 1.74 & 1.73 \\
\hline System 1 & $0.0752-2$ & 0.075 & 2 & 2 & 5-Dec & 74 & 1.73 & 1.72 & 1.72 & 1.72 \\
\hline System 1 & $0.0752-3$ & 0.075 & 2 & 3 & 14-Nov & 73 & 1.71 & 1.71 & 1.71 & 1.71 \\
\hline System 1 & $0.0752-3$ & 0.075 & 2 & 3 & 19-Nov & 73 & 1.73 & 1.72 & 1.73 & 1.73 \\
\hline System 1 & $0.0752-3$ & 0.075 & 2 & 3 & 20-Nov & 63 & 1.74 & 1.73 & 1.74 & 1.73 \\
\hline System 1 & $0.0752-3$ & 0.075 & 2 & 3 & 21-Nov & 70 & 1.72 & 1.73 & 1.73 & 1.73 \\
\hline System 1 & $0.0752-3$ & 0.075 & 2 & 3 & 26-Nov & 70 & 1.74 & 1.74 & 1.74 & 1.74 \\
\hline System 1 & $0.0752-3$ & 0.075 & 2 & 3 & 5-Dec & 74 & 1.71 & 1.72 & 1.71 & 1.72 \\
\hline System 1 & $0.0753-1$ & 0.075 & 3 & 1 & 14-Nov & 73 & 1.71 & 1.71 & 1.71 & 1.72 \\
\hline System 1 & $0.0753-1$ & 0.075 & 3 & 1 & 19-Nov & 73 & 1.71 & 1.72 & 1.73 & 1.72 \\
\hline System 1 & $0.0753-1$ & 0.075 & 3 & 1 & 20-Nov & 63 & 1.72 & 1.73 & 1.73 & 1.74 \\
\hline System 1 & $0.0753-1$ & 0.075 & 3 & 1 & 21-Nov & 70 & 1.71 & 1.73 & 1.73 & 1.73 \\
\hline System 1 & $0.0753-1$ & 0.075 & 3 & 1 & 26-Nov & 70 & 1.72 & 1.74 & 1.75 & 1.72 \\
\hline System 1 & $0.0753-1$ & 0.075 & 3 & 1 & 5-Dec & 74 & 1.71 & 1.71 & 1.72 & 1.72 \\
\hline System 1 & $0.0753-2$ & 0.075 & 3 & 2 & 14-Nov & 73 & 1.71 & 1.72 & 1.72 & 1.72 \\
\hline System 1 & $0.0753-2$ & 0.075 & 3 & 2 & 19-Nov & 73 & 1.73 & 1.72 & 1.74 & 1.74 \\
\hline System 1 & $0.0753-2$ & 0.075 & 3 & 2 & 20-Nov & 63 & 1.73 & 1.73 & 1.74 & 1.73 \\
\hline System 1 & $0.0753-2$ & 0.075 & 3 & 2 & 21-Nov & 70 & 1.74 & 1.73 & 1.75 & 1.74 \\
\hline System 1 & $0.0753-2$ & 0.075 & 3 & 2 & 26-Nov & 70 & 1.74 & 1.74 & 1.75 & 1.75 \\
\hline System 1 & $0.0753-2$ & 0.075 & 3 & 2 & 5-Dec & 74 & 1.71 & 1.7 & 1.72 & 1.71 \\
\hline System 1 & $0.0753-3$ & 0.075 & 3 & 3 & 14-Nov & 73 & 1.7 & 1.71 & 1.72 & 1.72 \\
\hline System 1 & $0.0753-3$ & 0.075 & 3 & 3 & 19-Nov & 73 & 1.72 & 1.71 & 1.72 & 1.72 \\
\hline System 1 & $0.0753-3$ & 0.075 & 3 & 3 & 20-Nov & 63 & 1.72 & 1.72 & 1.73 & 1.72 \\
\hline System 1 & $0.0753-3$ & 0.075 & 3 & 3 & 21-Nov & 70 & 1.71 & 1.72 & 1.73 & 1.72 \\
\hline System 1 & $0.0753-3$ & 0.075 & 3 & 3 & 26-Nov & 70 & 1.73 & 1.73 & 1.74 & 1.73 \\
\hline System 1 & $0.0753-3$ & 0.075 & 3 & 3 & 5-Dec & 74 & 1.71 & 1.71 & 1.72 & 1.71 \\
\hline System 1 & $0.11-1$ & 0.1 & 1 & 1 & 14-Nov & 73 & 2.02 & 2.03 & 2.03 & 2 \\
\hline System 1 & $0.11-1$ & 0.1 & 1 & 1 & 19-Nov & 73 & 2.04 & 2.04 & 2.03 & 2.02 \\
\hline System 1 & $0.11-1$ & 0.1 & 1 & 1 & 20-Nov & 63 & 2.02 & 2.05 & 2.04 & 2.02 \\
\hline System 1 & $0.11-1$ & 0.1 & 1 & 1 & 21-Nov & 70 & 2.04 & 2.05 & 2.05 & 2.03 \\
\hline System 1 & $0.11-1$ & 0.1 & 1 & 1 & 26-Nov & 70 & 2.05 & 2.05 & 2.05 & 2.03 \\
\hline System 1 & $0.11-1$ & 0.1 & 1 & 1 & 5-Dec & 74 & 2.02 & 2.03 & 2.03 & 2.02 \\
\hline System 1 & $0.11-2$ & 0.1 & 1 & 2 & 14-Nov & 73 & 2.01 & 2.02 & 1.97 & 2 \\
\hline System 1 & $0.11-2$ & 0.1 & 1 & 2 & 19-Nov & 73 & 2.02 & 2.04 & 1.98 & 2.01 \\
\hline System 1 & $0.11-2$ & 0.1 & 1 & 2 & 20-Nov & 63 & 2.02 & 2.05 & 2 & 2.02 \\
\hline System 1 & $0.11-2$ & 0.1 & 1 & 2 & 21-Nov & 70 & 2.01 & 2.05 & 1.99 & 2.02 \\
\hline System 1 & $0.11-2$ & 0.1 & 1 & 2 & 26-Nov & 70 & 2.03 & 2.06 & 2.01 & 2.02 \\
\hline System 1 & $0.11-2$ & 0.1 & 1 & 2 & 5-Dec & 74 & 2.02 & 2.02 & 1.98 & 2 \\
\hline
\end{tabular}


Table A1. Concentration Unit (CU) Measurements

\begin{tabular}{|c|c|c|c|c|c|c|c|c|c|c|}
\hline \multirow{2}{*}{ Meter } & \multirow{2}{*}{$\begin{array}{l}\text { Peanut } \\
\text { Vial ID }\end{array}$} & \multirow{2}{*}{ Wt\% } & \multirow{2}{*}{ Bottle } & \multirow{2}{*}{ Run } & \multirow{2}{*}{ Date } & \multirow{2}{*}{ Temp } & & ncentratio & Jnits ( & \\
\hline & & & & & & & Back & Forward & Left & Right \\
\hline System 1 & $0.11-3$ & 0.1 & 1 & 3 & 14-Nov & 73 & 1.99 & 2 & 2.01 & 2.01 \\
\hline System 1 & $0.11-3$ & 0.1 & 1 & 3 & 19-Nov & 73 & 2 & 2 & 2 & 2.01 \\
\hline System 1 & $0.11-3$ & 0.1 & 1 & 3 & 20-Nov & 63 & 2 & 2 & 2.02 & 2.01 \\
\hline System 1 & $0.11-3$ & 0.1 & 1 & 3 & 21-Nov & 70 & 2.01 & 2.01 & 2.01 & 2 \\
\hline System 1 & $0.11-3$ & 0.1 & 1 & 3 & 26-Nov & 70 & 2.02 & 2.02 & 2.02 & 2.02 \\
\hline System 1 & $0.11-3$ & 0.1 & 1 & 3 & 5-Dec & 74 & 1.98 & 1.99 & 2.01 & 2 \\
\hline System 1 & $0.12-1$ & 0.1 & 2 & 1 & 14-Nov & 73 & 2 & 2.01 & 1.98 & 1.98 \\
\hline System 1 & $0.12-1$ & 0.1 & 2 & 1 & 19-Nov & 73 & 2.01 & 2.03 & 2 & 2 \\
\hline System 1 & $0.12-1$ & 0.1 & 2 & 1 & 20-Nov & 63 & 2.04 & 2.04 & 2.02 & 2.02 \\
\hline System 1 & $0.12-1$ & 0.1 & 2 & 1 & 21-Nov & 70 & 2.04 & 2.04 & 2.03 & 2.02 \\
\hline System 1 & $0.12-1$ & 0.1 & 2 & 1 & 26-Nov & 70 & 2.04 & 2.05 & 2.04 & 2.03 \\
\hline System 1 & $0.12-1$ & 0.1 & 2 & 1 & 5-Dec & 74 & 2.03 & 2.03 & 2.02 & 2.02 \\
\hline System 1 & $0.12-2$ & 0.1 & 2 & 2 & 14-Nov & 73 & 2.01 & 2.02 & 2.02 & 2.01 \\
\hline System 1 & $0.12-2$ & 0.1 & 2 & 2 & 19-Nov & 73 & 2.03 & 2.03 & 2.03 & 2.03 \\
\hline System 1 & $0.12-2$ & 0.1 & 2 & 2 & 20-Nov & 63 & 2.04 & 2.03 & 2.04 & 2.03 \\
\hline System 1 & $0.12-2$ & 0.1 & 2 & 2 & 21-Nov & 70 & 2.04 & 2.04 & 2.04 & 2.04 \\
\hline System 1 & $0.12-2$ & 0.1 & 2 & 2 & 26-Nov & 70 & 2.04 & 2.05 & 2.05 & 2.04 \\
\hline System 1 & $0.12-2$ & 0.1 & 2 & 2 & 5-Dec & 74 & 2.04 & 2.03 & 2.05 & 2.05 \\
\hline System 1 & $0.12-3$ & 0.1 & 2 & 3 & 14-Nov & 73 & 2.03 & 2.03 & 2.01 & 2.01 \\
\hline System 1 & $0.12-3$ & 0.1 & 2 & 3 & 19-Nov & 73 & 2.04 & 2.05 & 2.02 & 2.03 \\
\hline System 1 & $0.12-3$ & 0.1 & 2 & 3 & 20-Nov & 63 & 2.05 & 2.07 & 2.04 & 2.03 \\
\hline System 1 & $0.12-3$ & 0.1 & 2 & 3 & 21-Nov & 70 & 2.05 & 2.06 & 2.03 & 2.03 \\
\hline System 1 & $0.12-3$ & 0.1 & 2 & 3 & 26-Nov & 70 & 2.06 & 2.08 & 2.04 & 2.04 \\
\hline System 1 & $0.12-3$ & 0.1 & 2 & 3 & 5-Dec & 74 & 2.06 & 2.05 & 2.03 & 2.03 \\
\hline System 1 & $0.13-1$ & 0.1 & 3 & 1 & 14-Nov & 73 & 2 & 2 & 1.99 & 1.97 \\
\hline System 1 & $0.13-1$ & 0.1 & 3 & 1 & 19-Nov & 73 & 1.99 & 2.01 & 1.99 & 2 \\
\hline System 1 & $0.13-1$ & 0.1 & 3 & 1 & 20-Nov & 63 & 2 & 2.02 & 2.01 & 2.01 \\
\hline System 1 & $0.13-1$ & 0.1 & 3 & 1 & 21-Nov & 70 & 2.01 & 2.01 & 2 & 2 \\
\hline System 1 & $0.13-1$ & 0.1 & 3 & 1 & 26-Nov & 70 & 2.02 & 2.03 & 2.02 & 2.01 \\
\hline System 1 & $0.13-1$ & 0.1 & 3 & 1 & 5-Dec & 74 & 2.01 & 2 & 2 & 2.01 \\
\hline System 1 & $0.13-2$ & 0.1 & 3 & 2 & 14-Nov & 73 & 2 & 2.01 & 2.02 & 2.02 \\
\hline System 1 & $0.13-2$ & 0.1 & 3 & 2 & 19-Nov & 73 & 2.01 & 2.02 & 2.02 & 2.02 \\
\hline System 1 & $0.13-2$ & 0.1 & 3 & 2 & 20 -Nov & 63 & 2.04 & 2.04 & 2.05 & 2.05 \\
\hline System 1 & $0.13-2$ & 0.1 & 3 & 2 & 21-Nov & 70 & 2.03 & 2.05 & 2.05 & 2.04 \\
\hline System 1 & $0.13-2$ & 0.1 & 3 & 2 & 26-Nov & 70 & 2.04 & 2.04 & 2.06 & 2.05 \\
\hline System 1 & $0.13-2$ & 0.1 & 3 & 2 & 5-Dec & 74 & 2.02 & 2.02 & 2.03 & 2.02 \\
\hline System 1 & $0.13-3$ & 0.1 & 3 & 3 & 14-Nov & 73 & 1.99 & 1.99 & 1.99 & 2 \\
\hline System 1 & $0.13-3$ & 0.1 & 3 & 3 & 19-Nov & 73 & 1.98 & 2 & 2 & 2.01 \\
\hline System 1 & $0.13-3$ & 0.1 & 3 & 3 & 20-Nov & 63 & 1.98 & 2.01 & 2.01 & 2.02 \\
\hline System 1 & $0.13-3$ & 0.1 & 3 & 3 & 21-Nov & 70 & 1.99 & 2 & 2.01 & 2 \\
\hline System 1 & $0.13-3$ & 0.1 & 3 & 3 & 26-Nov & 70 & 2 & 2.02 & 2.02 & 2.02 \\
\hline System 1 & $0.13-3$ & 0.1 & 3 & 3 & 5-Dec & 74 & 2 & 2.01 & 2.02 & 2.02 \\
\hline System 1 & $0.141-1$ & 0.14 & 1 & 1 & 14-Nov & 73 & 2.35 & 2.36 & 2.35 & 2.33 \\
\hline System 1 & $0.141-1$ & 0.14 & 1 & 1 & 19-Nov & 73 & 2.38 & 2.38 & 2.36 & 2.35 \\
\hline System 1 & $0.141-1$ & 0.14 & 1 & 1 & 20-Nov & 63 & 2.39 & 2.4 & 2.38 & 2.37 \\
\hline System 1 & $0.141-1$ & 0.14 & 1 & 1 & 21-Nov & 70 & 2.39 & 2.4 & 2.39 & 2.38 \\
\hline System 1 & $0.141-1$ & 0.14 & 1 & 1 & 26-Nov & 70 & 2.41 & 2.4 & 2.39 & 2.38 \\
\hline System 1 & $0.141-1$ & 0.14 & 1 & 1 & 5-Dec & 74 & 2.39 & 2.4 & 2.37 & 2.35 \\
\hline System 1 & $0.141-2$ & 0.14 & 1 & 2 & 14-Nov & 73 & 2.33 & 2.34 & 2.33 & 2.32 \\
\hline System 1 & $0.14 \quad 1-2$ & 0.14 & 1 & 2 & 19-Nov & 73 & 2.35 & 2.37 & 2.37 & 2.35 \\
\hline System 1 & $0.141-2$ & 0.14 & 1 & 2 & 20-Nov & 63 & 2.37 & 2.38 & 2.38 & 2.36 \\
\hline System 1 & $0.14 \quad 1-2$ & 0.14 & 1 & 2 & 21-Nov & 70 & 2.36 & 2.38 & 2.38 & 2.35 \\
\hline System 1 & $0.141-2$ & 0.14 & 1 & 2 & 26-Nov & 70 & 2.37 & 2.39 & 2.39 & 2.37 \\
\hline System 1 & $0.141-2$ & 0.14 & 1 & 2 & 5-Dec & 74 & 2.35 & 2.38 & 2.36 & 2.33 \\
\hline System 1 & $0.141-3$ & 0.14 & 1 & 3 & 14-Nov & 73 & 2.36 & 2.36 & 2.33 & 2.32 \\
\hline System 1 & $0.141-3$ & 0.14 & 1 & 3 & 19-Nov & 73 & 2.37 & 2.37 & 2.35 & 2.34 \\
\hline System 1 & $0.14 \quad 1-3$ & 0.14 & 1 & 3 & 20-Nov & 63 & 2.38 & 2.39 & 2.37 & 2.34 \\
\hline System 1 & $0.141-3$ & 0.14 & 1 & 3 & 21-Nov & 70 & 2.38 & 2.4 & 2.36 & 2.34 \\
\hline System 1 & $0.14 \quad 1-3$ & 0.14 & 1 & 3 & 26-Nov & 70 & 2.39 & 2.41 & 2.39 & 2.36 \\
\hline System 1 & $0.141-3$ & 0.14 & 1 & 3 & 5-Dec & 74 & 2.37 & 2.37 & 2.34 & 2.33 \\
\hline System 1 & $0.142-1$ & 0.14 & 2 & 1 & 14-Nov & 73 & 2.32 & 2.34 & 2.34 & 2.34 \\
\hline System 1 & $0.142-1$ & 0.14 & 2 & 1 & 19-Nov & 73 & 2.35 & 2.36 & 2.36 & 2.36 \\
\hline System 1 & $0.142-1$ & 0.14 & 2 & 1 & 20-Nov & 63 & 2.36 & 2.38 & 2.38 & 2.37 \\
\hline System 1 & $0.142-1$ & 0.14 & 2 & 1 & 21-Nov & 70 & 2.36 & 2.37 & 2.38 & 2.37 \\
\hline System 1 & $0.142-1$ & 0.14 & 2 & 1 & 26-Nov & 70 & 2.37 & 2.38 & 2.37 & 2.38 \\
\hline System 1 & $0.142-1$ & 0.14 & 2 & 1 & 5-Dec & 74 & 2.35 & 2.35 & 2.35 & 2.35 \\
\hline System 1 & $0.142-2$ & 0.14 & 2 & 2 & 14-Nov & 73 & 2.32 & 2.33 & 2.32 & 2.31 \\
\hline System 1 & $0.142-2$ & 0.14 & 2 & 2 & 19-Nov & 73 & 2.33 & 2.35 & 2.34 & 2.32 \\
\hline System 1 & $0.142-2$ & 0.14 & 2 & 2 & 20-Nov & 63 & 2.35 & 2.36 & 2.35 & 2.34 \\
\hline System 1 & $0.142-2$ & 0.14 & 2 & 2 & 21-Nov & 70 & 2.34 & 2.35 & 2.34 & 2.34 \\
\hline System 1 & $0.142-2$ & 0.14 & 2 & 2 & 26-Nov & 70 & 2.35 & 2.36 & 2.35 & 2.34 \\
\hline
\end{tabular}


Table A1. Concentration Unit (CU) Measurements

\begin{tabular}{|c|c|c|c|c|c|c|c|c|c|c|}
\hline \multirow{2}{*}{ Meter } & \multirow{2}{*}{$\begin{array}{l}\text { Peanut } \\
\text { Vial ID } \\
\end{array}$} & \multirow{2}{*}{ Wt\% } & \multirow{2}{*}{ Bottle } & \multirow{2}{*}{ Run } & \multirow{2}{*}{ Date } & \multirow{2}{*}{ Temp } & & ncentratio & Jnits ( & \\
\hline & & & & & & & Back & Forward & Left & Right \\
\hline System 1 & $0.142-2$ & 0.14 & 2 & 2 & 5-Dec & 74 & 2.35 & 2.34 & 2.34 & 2.32 \\
\hline System 1 & $0.142-3$ & 0.14 & 2 & 3 & 14-Nov & 73 & 2.36 & 2.37 & 2.35 & 2.35 \\
\hline System 1 & $0.142-3$ & 0.14 & 2 & 3 & 19-Nov & 73 & 2.39 & 2.4 & 2.38 & 2.38 \\
\hline System 1 & $0.142-3$ & 0.14 & 2 & 3 & 20-Nov & 63 & 2.39 & 2.39 & 2.41 & 2.38 \\
\hline System 1 & $0.142-3$ & 0.14 & 2 & 3 & 21-Nov & 70 & 2.38 & 2.39 & 2.4 & 2.4 \\
\hline System 1 & $0.142-3$ & 0.14 & 2 & 3 & 26-Nov & 70 & 2.39 & 2.41 & 2.4 & 2.41 \\
\hline System 1 & $0.142-3$ & 0.14 & 2 & 3 & 5-Dec & 74 & 2.37 & 2.39 & 2.36 & 2.38 \\
\hline System 1 & $0.143-1$ & 0.14 & 3 & 1 & 14-Nov & 73 & 2.33 & 2.35 & 2.37 & 2.37 \\
\hline System 1 & $0.143-1$ & 0.14 & 3 & 1 & 19-Nov & 73 & 2.36 & 2.37 & 2.39 & 2.39 \\
\hline System 1 & $0.143-1$ & 0.14 & 3 & 1 & 20-Nov & 63 & 2.37 & 2.38 & 2.4 & 2.4 \\
\hline System 1 & $0.143-1$ & 0.14 & 3 & 1 & 21-Nov & 70 & 2.38 & 2.4 & 2.39 & 2.38 \\
\hline System 1 & $0.143-1$ & 0.14 & 3 & 1 & 26-Nov & 70 & 2.38 & 2.39 & 2.41 & 2.4 \\
\hline System 1 & $0.143-1$ & 0.14 & 3 & 1 & 5-Dec & 74 & 2.35 & 2.37 & 2.38 & 2.38 \\
\hline System 1 & $0.143-2$ & 0.14 & 3 & 2 & 14-Nov & 73 & 2.36 & 2.37 & 2.36 & 2.36 \\
\hline System 1 & $0.143-2$ & 0.14 & 3 & 2 & 19-Nov & 73 & 2.38 & 2.39 & 2.39 & 2.38 \\
\hline System 1 & $0.143-2$ & 0.14 & 3 & 2 & 20-Nov & 63 & 2.4 & 2.41 & 2.41 & 2.4 \\
\hline System 1 & $0.143-2$ & 0.14 & 3 & 2 & 21-Nov & 70 & 2.38 & 2.39 & 2.39 & 2.39 \\
\hline System 1 & $0.143-2$ & 0.14 & 3 & 2 & 26-Nov & 70 & 2.4 & 2.41 & 2.4 & 2.39 \\
\hline System 1 & $0.143-2$ & 0.14 & 3 & 2 & 5-Dec & 74 & 2.39 & 2.39 & 2.38 & 2.38 \\
\hline System 1 & $0.143-3$ & 0.14 & 3 & 3 & 14-Nov & 73 & 2.36 & 2.36 & 2.36 & 2.35 \\
\hline System 1 & $0.143-3$ & 0.14 & 3 & 3 & 19-Nov & 73 & 2.38 & 2.38 & 2.38 & 2.37 \\
\hline System 1 & $0.143-3$ & 0.14 & 3 & 3 & 20-Nov & 63 & 2.39 & 2.39 & 2.39 & 2.39 \\
\hline System 1 & $0.143-3$ & 0.14 & 3 & 3 & 21-Nov & 70 & 2.39 & 2.4 & 2.39 & 2.38 \\
\hline System 1 & $0.143-3$ & 0.14 & 3 & 3 & 26-Nov & 70 & 2.39 & 2.4 & 2.4 & 2.39 \\
\hline System 1 & $0.143-3$ & 0.14 & 3 & 3 & 5-Dec & 74 & 2.37 & 2.39 & 2.38 & 2.38 \\
\hline System 1 & 0.15 & 0.15 & 1 & 1 & 14-Nov & 73 & 2.43 & 2.42 & 2.43 & 2.43 \\
\hline System 1 & 0.15 & 0.15 & 1 & 1 & 19-Nov & 73 & 2.45 & 2.43 & 2.43 & 2.45 \\
\hline System 1 & 0.15 & 0.15 & 1 & 1 & 20-Nov & 63 & 2.46 & 2.45 & 2.45 & 2.46 \\
\hline System 1 & 0.15 & 0.15 & 1 & 1 & 21-Nov & 70 & 2.46 & 2.45 & 2.44 & 2.46 \\
\hline System 1 & 0.15 & 0.15 & 1 & 1 & 26-Nov & 70 & 2.47 & 2.47 & 2.45 & 2.46 \\
\hline System 1 & 0.15 & 0.15 & 1 & 1 & 5-Dec & 74 & 2.46 & 2.43 & 2.45 & 2.46 \\
\hline System 1 & $0.21-1$ & 0.2 & 1 & 1 & 14-Nov & 73 & 2.7 & 2.7 & 2.71 & 2.71 \\
\hline System 1 & $0.21-1$ & 0.2 & 1 & 1 & 19-Nov & 73 & 2.71 & 2.72 & 2.73 & 2.73 \\
\hline System 1 & $0.21-1$ & 0.2 & 1 & 1 & 20-Nov & 63 & 2.72 & 2.73 & 2.74 & 2.74 \\
\hline System 1 & $0.21-1$ & 0.2 & 1 & 1 & 21-Nov & 70 & 2.71 & 2.72 & 2.74 & 2.73 \\
\hline System 1 & $0.21-1$ & 0.2 & 1 & 1 & 26-Nov & 70 & 2.71 & 2.73 & 2.75 & 2.72 \\
\hline System 1 & $0.21-1$ & 0.2 & 1 & 1 & 5-Dec & 74 & 2.72 & 2.72 & 2.73 & 2.73 \\
\hline System 1 & $0.21-2$ & 0.2 & 1 & 2 & 14-Nov & 73 & 2.69 & 2.69 & 2.69 & 2.69 \\
\hline System 1 & $0.21-2$ & 0.2 & 1 & 2 & 19-Nov & 73 & 2.7 & 2.71 & 2.71 & 2.7 \\
\hline System 1 & $0.21-2$ & 0.2 & 1 & 2 & 20-Nov & 63 & 2.71 & 2.72 & 2.7 & 2.71 \\
\hline System 1 & $0.21-2$ & 0.2 & 1 & 2 & 21-Nov & 70 & 2.71 & 2.72 & 2.71 & 2.71 \\
\hline System 1 & $0.21-2$ & 0.2 & 1 & 2 & 26-Nov & 70 & 2.72 & 2.72 & 2.71 & 2.72 \\
\hline System 1 & $0.21-2$ & 0.2 & 1 & 2 & 5-Dec & 74 & 2.71 & 2.7 & 2.7 & 2.7 \\
\hline System 1 & $0.21-3$ & 0.2 & 1 & 3 & 14-Nov & 73 & 2.72 & 2.72 & 2.69 & 2.69 \\
\hline System 1 & $0.21-3$ & 0.2 & 1 & 3 & 19-Nov & 73 & 2.74 & 2.71 & 2.71 & 2.71 \\
\hline System 1 & $0.21-3$ & 0.2 & 1 & 3 & 20-Nov & 63 & 2.75 & 2.75 & 2.72 & 2.72 \\
\hline System 1 & $0.21-3$ & 0.2 & 1 & 3 & 21-Nov & 70 & 2.75 & 2.75 & 2.72 & 2.72 \\
\hline System 1 & $0.21-3$ & 0.2 & 1 & 3 & 26-Nov & 70 & 2.77 & 2.76 & 2.73 & 2.73 \\
\hline System 1 & $0.21-3$ & 0.2 & 1 & 3 & 5-Dec & 74 & 2.75 & 2.75 & 2.72 & 2.73 \\
\hline System 1 & $0.22-1$ & 0.2 & 2 & 1 & 14-Nov & 73 & 2.69 & 2.7 & 2.71 & 2.71 \\
\hline System 1 & $0.22-1$ & 0.2 & 2 & 1 & 19-Nov & 73 & 2.73 & 2.73 & 2.73 & 2.73 \\
\hline System 1 & $0.22-1$ & 0.2 & 2 & 1 & 20-Nov & 63 & 2.74 & 2.74 & 2.74 & 2.73 \\
\hline System 1 & $0.22-1$ & 0.2 & 2 & 1 & 21-Nov & 70 & 2.74 & 2.73 & 2.74 & 2.74 \\
\hline System 1 & $0.22-1$ & 0.2 & 2 & 1 & 26-Nov & 70 & 2.75 & 2.74 & 2.76 & 2.75 \\
\hline System 1 & $0.22-1$ & 0.2 & 2 & 1 & 5-Dec & 74 & 2.73 & 2.74 & 2.75 & 2.74 \\
\hline System 1 & $0.22-2$ & 0.2 & 2 & 2 & 14-Nov & 73 & 2.71 & 2.71 & 2.69 & 2.69 \\
\hline System 1 & $0.22-2$ & 0.2 & 2 & 2 & 19-Nov & 73 & 2.74 & 2.74 & 2.72 & 2.72 \\
\hline System 1 & $0.22-2$ & 0.2 & 2 & 2 & 20-Nov & 63 & 2.75 & 2.75 & 2.73 & 2.73 \\
\hline System 1 & $0.22-2$ & 0.2 & 2 & 2 & 21-Nov & 70 & 2.75 & 2.76 & 2.73 & 2.73 \\
\hline System 1 & $0.22-2$ & 0.2 & 2 & 2 & 26-Nov & 70 & 2.76 & 2.76 & 2.74 & 2.75 \\
\hline System 1 & $0.22-2$ & 0.2 & 2 & 2 & 5-Dec & 74 & 2.76 & 2.76 & 2.75 & 2.75 \\
\hline System 1 & $0.22-3$ & 0.2 & 2 & 3 & 14-Nov & 73 & 2.7 & 2.7 & 2.72 & 2.72 \\
\hline System 1 & $0.22-3$ & 0.2 & 2 & 3 & 19-Nov & 73 & 2.73 & 2.73 & 2.75 & 2.75 \\
\hline System 1 & $0.22-3$ & 0.2 & 2 & 3 & 20-Nov & 63 & 2.73 & 2.74 & 2.76 & 2.76 \\
\hline System 1 & $0.22-3$ & 0.2 & 2 & 3 & 21-Nov & 70 & 2.73 & 2.74 & 2.76 & 2.75 \\
\hline System 1 & $0.22-3$ & 0.2 & 2 & 3 & 26-Nov & 70 & 2.73 & 2.74 & 2.76 & 2.76 \\
\hline System 1 & $0.22-3$ & 0.2 & 2 & 3 & 5-Dec & 74 & 2.74 & 2.74 & 2.77 & 2.76 \\
\hline System 1 & $0.23-1$ & 0.2 & 3 & 1 & 14-Nov & 73 & 2.69 & 2.7 & 2.7 & 2.71 \\
\hline System 1 & $0.23-1$ & 0.2 & 3 & 1 & 19-Nov & 73 & 2.73 & 2.73 & 2.74 & 2.74 \\
\hline System 1 & $0.23-1$ & 0.2 & 3 & 1 & 20-Nov & 63 & 2.74 & 2.74 & 2.75 & 2.74 \\
\hline System 1 & $0.23-1$ & 0.2 & 3 & 1 & 21-Nov & 70 & 2.74 & 2.75 & 2.75 & 2.75 \\
\hline
\end{tabular}


Table A1. Concentration Unit (CU) Measurements

\begin{tabular}{|c|c|c|c|c|c|c|c|c|c|c|}
\hline \multirow{2}{*}{ Meter } & \multirow{2}{*}{$\begin{array}{l}\text { Peanut } \\
\text { Vial ID }\end{array}$} & \multirow{2}{*}{ Wt\% } & \multirow{2}{*}{ Bottle } & \multirow{2}{*}{ Run } & \multirow{2}{*}{ Date } & \multirow{2}{*}{ Temp } & & oncentratior & Jnits ( & \\
\hline & & & & & & & Back & Forward & Left & Right \\
\hline System 1 & $0.23-1$ & 0.2 & 3 & 1 & 26-Nov & 70 & 2.75 & 2.76 & 2.76 & 2.76 \\
\hline System 1 & $0.23-1$ & 0.2 & 3 & 1 & 5-Dec & 74 & 2.71 & 2.75 & 2.76 & 2.76 \\
\hline System 1 & $0.23-2$ & 0.2 & 3 & 2 & 14-Nov & 73 & 2.69 & 2.7 & 2.68 & 2.69 \\
\hline System 1 & $0.23-2$ & 0.2 & 3 & 2 & 19-Nov & 73 & 2.71 & 2.72 & 2.7 & 2.7 \\
\hline System 1 & $0.23-2$ & 0.2 & 3 & 2 & 20-Nov & 63 & 2.72 & 2.74 & 2.71 & 2.72 \\
\hline System 1 & $0.23-2$ & 0.2 & 3 & 2 & 21-Nov & 70 & 2.71 & 2.73 & 2.71 & 2.71 \\
\hline System 1 & $0.23-2$ & 0.2 & 3 & 2 & 26-Nov & 70 & 2.73 & 2.74 & 2.72 & 2.73 \\
\hline System 1 & $0.23-2$ & 0.2 & 3 & 2 & 5-Dec & 74 & 2.72 & 2.73 & 2.7 & 2.71 \\
\hline System 1 & $0.23-3$ & 0.2 & 3 & 3 & 14-Nov & 73 & 2.67 & 2.67 & 2.68 & 2.69 \\
\hline System 1 & $0.23-3$ & 0.2 & 3 & 3 & 19-Nov & 73 & 2.68 & 2.68 & 2.7 & 2.69 \\
\hline System 1 & $0.23-3$ & 0.2 & 3 & 3 & 20-Nov & 63 & 2.69 & 2.69 & 2.71 & 2.71 \\
\hline System 1 & $0.23-3$ & 0.2 & 3 & 3 & 21-Nov & 70 & 2.68 & 2.68 & 2.7 & 2.7 \\
\hline System 1 & $0.23-3$ & 0.2 & 3 & 3 & 26-Nov & 70 & 2.68 & 2.68 & 2.69 & 2.7 \\
\hline System 1 & $0.23-3$ & 0.2 & 3 & 3 & 5-Dec & 74 & 2.69 & 2.69 & 2.71 & 2.71 \\
\hline System 1 & 0.3 & 0.3 & 1 & 1 & 14-Nov & 73 & 3.12 & 3.12 & 3.13 & 3.13 \\
\hline System 1 & 0.3 & 0.3 & 1 & 1 & 19-Nov & 73 & 3.14 & 3.16 & 3.16 & 3.16 \\
\hline System 1 & 0.3 & 0.3 & 1 & 1 & 20-Nov & 63 & 3.16 & 3.17 & 3.18 & 3.17 \\
\hline System 1 & 0.3 & 0.3 & 1 & 1 & 21-Nov & 70 & 3.17 & 3.17 & 3.18 & 3.17 \\
\hline System 1 & 0.3 & 0.3 & 1 & 1 & 26-Nov & 70 & 3.16 & 3.18 & 3.19 & 3.18 \\
\hline System 1 & 0.3 & 0.3 & 1 & 1 & 5-Dec & 74 & 3.17 & 3.18 & 3.18 & 3.18 \\
\hline System 1 & 0.4 & 0.4 & 1 & 1 & 14-Nov & 73 & 3.44 & 3.43 & 3.46 & 3.46 \\
\hline System 1 & 0.4 & 0.4 & 1 & 1 & 19-Nov & 73 & 3.45 & 3.46 & 3.49 & 3.48 \\
\hline System 1 & 0.4 & 0.4 & 1 & 1 & 20-Nov & 63 & 3.46 & 3.47 & 3.5 & 3.49 \\
\hline System 1 & 0.4 & 0.4 & 1 & 1 & 21-Nov & 70 & 3.46 & 3.46 & 3.49 & 3.49 \\
\hline System 1 & 0.4 & 0.4 & 1 & 1 & 26-Nov & 70 & 3.46 & 3.46 & 3.5 & 3.49 \\
\hline System 1 & 0.4 & 0.4 & 1 & 1 & 5-Dec & 74 & 3.47 & 3.46 & 3.49 & 3.49 \\
\hline System 1 & 0.5 & 0.5 & 1 & 1 & 14-Nov & 73 & 3.7 & 3.69 & 3.69 & 3.7 \\
\hline System 1 & 0.5 & 0.5 & 1 & 1 & 19-Nov & 73 & 3.74 & 3.73 & 3.73 & 3.74 \\
\hline System 1 & 0.5 & 0.5 & 1 & 1 & 20-Nov & 63 & 3.76 & 3.75 & 3.75 & 3.76 \\
\hline System 1 & 0.5 & 0.5 & 1 & 1 & 21-Nov & 70 & 3.75 & 3.74 & 3.75 & 3.75 \\
\hline System 1 & 0.5 & 0.5 & 1 & 1 & 26-Nov & 70 & 3.76 & 3.75 & 3.76 & 3.77 \\
\hline System 1 & 0.5 & 0.5 & 1 & 1 & 5-Dec & 74 & 3.78 & 3.77 & 3.76 & 3.77 \\
\hline System 1 & 0.6 & 0.6 & 1 & 1 & 14-Nov & 73 & 3.95 & 3.96 & 3.96 & 3.96 \\
\hline System 1 & 0.6 & 0.6 & 1 & 1 & 19-Nov & 73 & 3.99 & 4 & 3.99 & 4 \\
\hline System 1 & 0.6 & 0.6 & 1 & 1 & 20-Nov & 63 & 4.03 & 4.03 & 4.02 & 4.02 \\
\hline System 1 & 0.6 & 0.6 & 1 & 1 & 21-Nov & 70 & 4.02 & 4.04 & 4.02 & 4.01 \\
\hline System 1 & 0.6 & 0.6 & 1 & 1 & 26-Nov & 70 & 4.03 & 4.05 & 4.03 & 4.03 \\
\hline System 1 & 0.6 & 0.6 & 1 & 1 & 5-Dec & 74 & 4.04 & 4.05 & 4.03 & 4.04 \\
\hline System 1 & 0.7 & 0.7 & 1 & 1 & 14-Nov & 73 & 4.18 & 4.13 & 4.17 & 4.18 \\
\hline System 1 & 0.7 & 0.7 & 1 & 1 & 19-Nov & 73 & 4.23 & 4.17 & 4.22 & 4.23 \\
\hline System 1 & 0.7 & 0.7 & 1 & 1 & 20-Nov & 63 & 4.25 & 4.2 & 4.24 & 4.25 \\
\hline System 1 & 0.7 & 0.7 & 1 & 1 & 21-Nov & 70 & 4.24 & 4.2 & 4.24 & 4.25 \\
\hline System 1 & 0.7 & 0.7 & 1 & 1 & 26-Nov & 70 & 4.26 & 4.22 & 4.25 & 4.26 \\
\hline System 1 & 0.7 & 0.7 & 1 & 1 & 5-Dec & 74 & 4.27 & 4.22 & 4.26 & 4.26 \\
\hline System 1 & 0.8 & 0.8 & 1 & 1 & 14-Nov & 73 & 4.41 & 4.4 & 4.43 & 4.42 \\
\hline System 1 & 0.8 & 0.8 & 1 & 1 & 19-Nov & 73 & 4.44 & 4.43 & 4.48 & 4.44 \\
\hline System 1 & 0.8 & 0.8 & 1 & 1 & 20-Nov & 63 & 4.46 & 4.45 & 4.48 & 4.45 \\
\hline System 1 & 0.8 & 0.8 & 1 & 1 & 21-Nov & 70 & 4.47 & 4.43 & 4.49 & 4.45 \\
\hline System 1 & 0.8 & 0.8 & 1 & 1 & 26-Nov & 70 & 4.48 & 4.45 & 4.5 & 4.46 \\
\hline System 1 & 0.8 & 0.8 & 1 & 1 & 5-Dec & 74 & 4.46 & 4.44 & 4.48 & 4.44 \\
\hline System 1 & 1.2 & 1.2 & 1 & 1 & 14-Nov & 73 & 4.8 & 4.89 & 4.85 & 4.87 \\
\hline System 1 & 1.2 & 1.2 & 1 & 1 & 19-Nov & 73 & 4.85 & 4.94 & 4.88 & 4.91 \\
\hline System 1 & 1.2 & 1.2 & 1 & 1 & 20-Nov & 63 & 4.86 & 4.95 & 4.94 & 4.92 \\
\hline System 1 & 1.2 & 1.2 & 1 & 1 & 21-Nov & 70 & 4.87 & 4.95 & 4.92 & 4.93 \\
\hline System 1 & 1.2 & 1.2 & 1 & 1 & 26-Nov & 70 & 4.87 & 4.97 & 4.94 & 4.96 \\
\hline System 1 & 1.2 & 1.2 & 1 & 1 & 5-Dec & 74 & 4.82 & 4.89 & 4.86 & 4.83 \\
\hline System 2 & DI 1-1 & 0 & 1 & 1 & 19-Nov & 73 & 0.09 & 0.09 & 0.07 & 0.07 \\
\hline System 2 & DI 1-1 & 0 & 1 & 1 & 20-Nov & 63 & 0.09 & 0.09 & 0.07 & 0.07 \\
\hline System 2 & DI 1-1 & 0 & 1 & 1 & 21-Nov & 70 & 0.09 & 0.09 & 0.07 & 0.07 \\
\hline System 2 & DI 1-1 & 0 & 1 & 1 & 26-Nov & 70 & 0.09 & 0.09 & 0.07 & 0.07 \\
\hline System 2 & DI $1-1$ & 0 & 1 & 1 & 27-Nov & 73 & 0.09 & 0.09 & 0.07 & 0.07 \\
\hline System 2 & DI 1-1 & 0 & 1 & 1 & 3-Dec & 73 & 0.1 & 0.08 & 0.07 & 0.07 \\
\hline System 2 & DI 1-1 & 0 & 1 & 1 & 4-Dec & 70 & 0.1 & 0.1 & 0.07 & 0.07 \\
\hline System 2 & DI 1-2 & 0 & 1 & 2 & 19-Nov & 73 & 0.11 & 0.12 & 0.09 & 0.08 \\
\hline System 2 & DI 1-2 & 0 & 1 & 2 & 20-Nov & 63 & 0.12 & 0.12 & 0.09 & 0.08 \\
\hline System 2 & DI $1-2$ & 0 & 1 & 2 & 21-Nov & 70 & 0.13 & 0.12 & 0.08 & 0.08 \\
\hline System 2 & DI 1-2 & 0 & 1 & 2 & 26-Nov & 70 & 0.12 & 0.12 & 0.08 & 0.08 \\
\hline System 2 & DI $1-2$ & 0 & 1 & 2 & 27-Nov & 73 & 0.13 & 0.12 & 0.08 & 0.08 \\
\hline System 2 & DI 1-2 & 0 & 1 & 2 & 3-Dec & 73 & 0.13 & 0.12 & 0.08 & 0.08 \\
\hline System 2 & DI 1-2 & 0 & 1 & 2 & 4-Dec & 70 & 0.13 & 0.13 & 0.08 & 0.08 \\
\hline System 2 & DI 1-3 & 0 & 1 & 3 & 19-Nov & 73 & 0.17 & 0.16 & 0.1 & 0.08 \\
\hline
\end{tabular}


Table A1. Concentration Unit (CU) Measurements

\begin{tabular}{|c|c|c|c|c|c|c|c|c|c|c|}
\hline \multirow{2}{*}{ Meter } & \multirow{2}{*}{$\begin{array}{l}\text { Peanut } \\
\text { Vial ID } \\
\end{array}$} & \multirow{2}{*}{ Wt\% } & \multirow{2}{*}{ Bottle } & \multirow{2}{*}{ Run } & \multirow{2}{*}{ Date } & \multirow{2}{*}{ Temp } & & oncentratio & Jnits ( & \\
\hline & & & & & & & Back & Forward & Left & Right \\
\hline System 2 & DI 1-3 & 0 & 1 & 3 & 20-Nov & 63 & 0.17 & 0.17 & 0.09 & 0.08 \\
\hline System 2 & DI 1-3 & 0 & 1 & 3 & 21-Nov & 70 & 0.17 & 0.16 & 0.1 & 0.09 \\
\hline System 2 & DI 1-3 & 0 & 1 & 3 & 26-Nov & 70 & 0.17 & 0.16 & 0.1 & 0.09 \\
\hline System 2 & DI $1-3$ & 0 & 1 & 3 & 27-Nov & 73 & 0.18 & 0.18 & 0.11 & 0.1 \\
\hline System 2 & DI 1-3 & 0 & 1 & 3 & 3-Dec & 73 & 0.18 & 0.17 & 0.11 & 0.1 \\
\hline System 2 & DI 1-3 & 0 & 1 & 3 & 4-Dec & 70 & 0.18 & 0.17 & 0.11 & 0.1 \\
\hline System 2 & $0.051-1$ & 0.05 & 1 & 1 & 19-Nov & 73 & 1.29 & 1.29 & 1.27 & 1.27 \\
\hline System 2 & $0.051-1$ & 0.05 & 1 & 1 & 20-Nov & 63 & 1.29 & 1.29 & 1.28 & 1.29 \\
\hline System 2 & $0.051-1$ & 0.05 & 1 & 1 & 21-Nov & 70 & 1.3 & 1.29 & 1.29 & 1.28 \\
\hline System 2 & $0.051-1$ & 0.05 & 1 & 1 & 26-Nov & 70 & 1.31 & 1.3 & 1.28 & 1.3 \\
\hline System 2 & $0.051-1$ & 0.05 & 1 & 1 & 27-Nov & 73 & 1.31 & 1.31 & 1.27 & 1.29 \\
\hline System 2 & $0.051-1$ & 0.05 & 1 & 1 & 3-Dec & 73 & 1.31 & 1.31 & 1.29 & 1.3 \\
\hline System 2 & $0.051-1$ & 0.05 & 1 & 1 & 4-Dec & 70 & 1.3 & 1.31 & 1.29 & 1.29 \\
\hline System 2 & $0.051-2$ & 0.05 & 1 & 2 & 19-Nov & 73 & 1.27 & 1.27 & 1.29 & 1.28 \\
\hline System 2 & $0.051-2$ & 0.05 & 1 & 2 & 20-Nov & 63 & 1.28 & 1.28 & 1.3 & 1.3 \\
\hline System 2 & $0.051-2$ & 0.05 & 1 & 2 & 21-Nov & 70 & 1.28 & 1.28 & 1.3 & 1.3 \\
\hline System 2 & $0.051-2$ & 0.05 & 1 & 2 & 26-Nov & 70 & 1.3 & 1.3 & 1.31 & 1.32 \\
\hline System 2 & $0.051-2$ & 0.05 & 1 & 2 & 27-Nov & 73 & 1.29 & 1.29 & 1.29 & 1.29 \\
\hline System 2 & $0.051-2$ & 0.05 & 1 & 2 & 3-Dec & 73 & 1.3 & 1.3 & 1.32 & 1.33 \\
\hline System 2 & $0.051-2$ & 0.05 & 1 & 2 & 4-Dec & 70 & 1.31 & 1.31 & 1.32 & 1.31 \\
\hline System 2 & $0.051-3$ & 0.05 & 1 & 3 & 19-Nov & 73 & 1.28 & 1.28 & 1.26 & 1.26 \\
\hline System 2 & $0.051-3$ & 0.05 & 1 & 3 & 20-Nov & 63 & 1.3 & 1.3 & 1.28 & 1.27 \\
\hline System 2 & $0.051-3$ & 0.05 & 1 & 3 & 21-Nov & 70 & 1.31 & 1.29 & 1.27 & 1.27 \\
\hline System 2 & $0.051-3$ & 0.05 & 1 & 3 & 26-Nov & 70 & 1.31 & 1.31 & 1.31 & 1.28 \\
\hline System 2 & $0.051-3$ & 0.05 & 1 & 3 & 27-Nov & 73 & 1.29 & 1.28 & 1.31 & 1.29 \\
\hline System 2 & $0.051-3$ & 0.05 & 1 & 3 & 3-Dec & 73 & 1.31 & 1.3 & 1.31 & 1.28 \\
\hline System 2 & $0.051-3$ & 0.05 & 1 & 3 & 4-Dec & 70 & 1.31 & 1.3 & 1.28 & 1.28 \\
\hline System 2 & $0.052-1$ & 0.05 & 2 & 1 & 19-Nov & 73 & 1.27 & 1.29 & 1.28 & 1.28 \\
\hline System 2 & $0.052-1$ & 0.05 & 2 & 1 & 20-Nov & 63 & 1.27 & 1.29 & 1.29 & 1.29 \\
\hline System 2 & $0.052-1$ & 0.05 & 2 & 1 & 21-Nov & 70 & 1.28 & 1.28 & 1.29 & 1.29 \\
\hline System 2 & $0.052-1$ & 0.05 & 2 & 1 & 26-Nov & 70 & 1.29 & 1.3 & 1.3 & 1.3 \\
\hline System 2 & $0.052-1$ & 0.05 & 2 & 1 & 27-Nov & 73 & 1.3 & 1.28 & 1.3 & 1.31 \\
\hline System 2 & $0.052-1$ & 0.05 & 2 & 1 & 3-Dec & 73 & 1.3 & 1.29 & 1.3 & 1.3 \\
\hline System 2 & $0.052-1$ & 0.05 & 2 & 1 & 4-Dec & 70 & 1.29 & 1.29 & 1.29 & 1.29 \\
\hline System 2 & $0.052-2$ & 0.05 & 2 & 2 & 19-Nov & 73 & 1.33 & 1.33 & 1.29 & 1.3 \\
\hline System 2 & $0.052-2$ & 0.05 & 2 & 2 & 20-Nov & 63 & 1.35 & 1.35 & 1.31 & 1.32 \\
\hline System 2 & $0.052-2$ & 0.05 & 2 & 2 & 21-Nov & 70 & 1.36 & 1.36 & 1.32 & 1.32 \\
\hline System 2 & $0.052-2$ & 0.05 & 2 & 2 & 26-Nov & 70 & 1.37 & 1.38 & 1.32 & 1.33 \\
\hline System 2 & $0.052-2$ & 0.05 & 2 & 2 & 27-Nov & 73 & 1.35 & 1.36 & 1.31 & 1.33 \\
\hline System 2 & $0.052-2$ & 0.05 & 2 & 2 & 3-Dec & 73 & 1.37 & 1.37 & 1.31 & 1.34 \\
\hline System 2 & $0.052-2$ & 0.05 & 2 & 2 & 4-Dec & 70 & 1.36 & 1.37 & 1.31 & 1.33 \\
\hline System 2 & $0.052-3$ & 0.05 & 2 & 3 & 19-Nov & 73 & 1.27 & 1.27 & 1.28 & 1.27 \\
\hline System 2 & $0.052-3$ & 0.05 & 2 & 3 & 20-Nov & 63 & 1.29 & 1.28 & 1.28 & 1.28 \\
\hline System 2 & $0.052-3$ & 0.05 & 2 & 3 & 21-Nov & 70 & 1.29 & 1.28 & 1.28 & 1.28 \\
\hline System 2 & $0.052-3$ & 0.05 & 2 & 3 & 26-Nov & 70 & 1.3 & 1.29 & 1.3 & 1.3 \\
\hline System 2 & $0.052-3$ & 0.05 & 2 & 3 & 27-Nov & 73 & 1.3 & 1.3 & 1.29 & 1.3 \\
\hline System 2 & $0.052-3$ & 0.05 & 2 & 3 & 3-Dec & 73 & 1.3 & 1.3 & 1.28 & 1.29 \\
\hline System 2 & $0.052-3$ & 0.05 & 2 & 3 & 4-Dec & 70 & 1.3 & 1.3 & 1.29 & 1.3 \\
\hline System 2 & $0.053-1$ & 0.05 & 3 & 1 & 19-Nov & 73 & 1.28 & 1.28 & 1.29 & 1.29 \\
\hline System 2 & $0.053-1$ & 0.05 & 3 & 1 & 20-Nov & 63 & 1.28 & 1.29 & 1.3 & 1.29 \\
\hline System 2 & $0.053-1$ & 0.05 & 3 & 1 & 21-Nov & 70 & 1.39 & 1.29 & 1.3 & 1.29 \\
\hline System 2 & $0.053-1$ & 0.05 & 3 & 1 & 26-Nov & 70 & 1.3 & 1.3 & 1.31 & 1.3 \\
\hline System 2 & $0.053-1$ & 0.05 & 3 & 1 & 27-Nov & 73 & 1.29 & 1.3 & 1.28 & 1.27 \\
\hline System 2 & $0.053-1$ & 0.05 & 3 & 1 & 3-Dec & 73 & 1.29 & 1.3 & 1.3 & 1.29 \\
\hline System 2 & $0.053-1$ & 0.05 & 3 & 1 & 4-Dec & 70 & 1.29 & 1.3 & 1.3 & 1.29 \\
\hline System 2 & $0.053-2$ & 0.05 & 3 & 2 & 19-Nov & 73 & 1.29 & 1.29 & 1.28 & 1.29 \\
\hline System 2 & $0.053-2$ & 0.05 & 3 & 2 & 20-Nov & 63 & 1.3 & 1.31 & 1.3 & 1.3 \\
\hline System 2 & $0.053-2$ & 0.05 & 3 & 2 & 21-Nov & 70 & 1.31 & 1.31 & 1.3 & 1.31 \\
\hline System 2 & $0.053-2$ & 0.05 & 3 & 2 & 26-Nov & 70 & 1.32 & 1.31 & 1.32 & 1.32 \\
\hline System 2 & $0.053-2$ & 0.05 & 3 & 2 & 27-Nov & 73 & 1.31 & 1.31 & 1.32 & 1.31 \\
\hline System 2 & $0.053-2$ & 0.05 & 3 & 2 & 3-Dec & 73 & 1.31 & 1.31 & 1.31 & 1.31 \\
\hline System 2 & $0.053-2$ & 0.05 & 3 & 2 & 4-Dec & 70 & 1.31 & 1.31 & 1.3 & 1.31 \\
\hline System 2 & $0.053-3$ & 0.05 & 3 & 3 & 19-Nov & 73 & 1.28 & 1.29 & 1.27 & 1.28 \\
\hline System 2 & $0.053-3$ & 0.05 & 3 & 3 & 20-Nov & 63 & 1.29 & 1.3 & 1.28 & 1.29 \\
\hline System 2 & $0.053-3$ & 0.05 & 3 & 3 & 21-Nov & 70 & 1.3 & 1.32 & 1.29 & 1.29 \\
\hline System 2 & $0.053-3$ & 0.05 & 3 & 3 & 26-Nov & 70 & 1.3 & 1.32 & 1.3 & 1.3 \\
\hline System 2 & $0.053-3$ & 0.05 & 3 & 3 & 27-Nov & 73 & 1.28 & 1.29 & 1.27 & 1.27 \\
\hline System 2 & $0.053-3$ & 0.05 & 3 & 3 & 3-Dec & 73 & 1.29 & 1.3 & 1.28 & 1.28 \\
\hline System 2 & $0.053-3$ & 0.05 & 3 & 3 & 4-Dec & 70 & 1.29 & 1.31 & 1.28 & 1.29 \\
\hline System 2 & $0.0751-1$ & 0.075 & 1 & 1 & 19-Nov & 73 & 1.67 & 1.66 & 1.68 & 1.68 \\
\hline System 2 & $0.0751-1$ & 0.075 & 1 & 1 & 20-Nov & 63 & 1.68 & 1.68 & 1.71 & 1.69 \\
\hline
\end{tabular}


Table A1. Concentration Unit (CU) Measurements

\begin{tabular}{|c|c|c|c|c|c|c|c|c|c|c|}
\hline \multirow{2}{*}{ Meter } & \multirow{2}{*}{$\begin{array}{l}\text { Peanut } \\
\text { Vial ID } \\
\end{array}$} & \multirow{2}{*}{ Wt\% } & \multirow{2}{*}{ Bottle } & \multirow{2}{*}{ Run } & \multirow{2}{*}{ Date } & \multirow{2}{*}{ Temp } & & oncentratio & Jnits ( & \\
\hline & & & & & & & Back & Forward & Left & Right \\
\hline System 2 & $0.0751-1$ & 0.075 & 1 & 1 & 21-Nov & 70 & 1.69 & 1.7 & 1.7 & 1.71 \\
\hline System 2 & $0.0751-1$ & 0.075 & 1 & 1 & 26-Nov & 70 & 1.7 & 1.7 & 1.73 & 1.71 \\
\hline System 2 & $0.0751-1$ & 0.075 & 1 & 1 & 27-Nov & 73 & 1.69 & 1.69 & 1.7 & 1.7 \\
\hline System 2 & $0.0751-1$ & 0.075 & 1 & 1 & 3-Dec & 73 & 1.7 & 1.7 & 1.71 & 1.7 \\
\hline System 2 & $0.0751-1$ & 0.075 & 1 & 1 & 4-Dec & 70 & 1.69 & 1.7 & 1.71 & 1.7 \\
\hline System 2 & $0.075 \quad 1-2$ & 0.075 & 1 & 2 & 19-Nov & 73 & 1.64 & 1.65 & 1.65 & 1.65 \\
\hline System 2 & $0.0751-2$ & 0.075 & 1 & 2 & 20-Nov & 63 & 1.66 & 1.67 & 1.67 & 1.67 \\
\hline System 2 & $0.075 \quad 1-2$ & 0.075 & 1 & 2 & 21-Nov & 70 & 1.68 & 1.67 & 1.69 & 1.67 \\
\hline System 2 & $0.0751-2$ & 0.075 & 1 & 2 & 26-Nov & 70 & 1.68 & 1.68 & 1.68 & 1.69 \\
\hline System 2 & $0.0751-2$ & 0.075 & 1 & 2 & 27-Nov & 73 & 1.67 & 1.67 & 1.67 & 1.67 \\
\hline System 2 & $0.0751-2$ & 0.075 & 1 & 2 & 3-Dec & 73 & 1.68 & 1.68 & 1.66 & 1.67 \\
\hline System 2 & $0.0751-2$ & 0.075 & 1 & 2 & 4-Dec & 70 & 1.68 & 1.67 & 1.68 & 1.68 \\
\hline System 2 & $0.075 \quad 1-3$ & 0.075 & 1 & 3 & 19-Nov & 73 & 1.69 & 1.68 & 1.67 & 1.69 \\
\hline System 2 & $0.0751-3$ & 0.075 & 1 & 3 & 20-Nov & 63 & 1.7 & 1.69 & 1.7 & 1.69 \\
\hline System 2 & $0.0751-3$ & 0.075 & 1 & 3 & 21-Nov & 70 & 1.7 & 1.7 & 1.69 & 1.69 \\
\hline System 2 & $0.0751-3$ & 0.075 & 1 & 3 & 26-Nov & 70 & 1.71 & 1.72 & 1.7 & 1.72 \\
\hline System 2 & $0.0751-3$ & 0.075 & 1 & 3 & 27-Nov & 73 & 1.71 & 1.72 & 1.7 & 1.7 \\
\hline System 2 & $0.0751-3$ & 0.075 & 1 & 3 & 3-Dec & 73 & 1.7 & 1.71 & 1.69 & 1.71 \\
\hline System 2 & $0.0751-3$ & 0.075 & 1 & 3 & 4-Dec & 70 & 1.71 & 1.7 & 1.69 & 1.7 \\
\hline System 2 & $0.0752-1$ & 0.075 & 2 & 1 & 19-Nov & 73 & 1.67 & 1.68 & 1.68 & 1.67 \\
\hline System 2 & $0.0752-1$ & 0.075 & 2 & 1 & 20-Nov & 63 & 1.7 & 1.72 & 1.7 & 1.7 \\
\hline System 2 & $0.0752-1$ & 0.075 & 2 & 1 & 21-Nov & 70 & 1.7 & 1.72 & 1.7 & 1.69 \\
\hline System 2 & $0.0752-1$ & 0.075 & 2 & 1 & 26-Nov & 70 & 1.71 & 1.73 & 1.72 & 1.71 \\
\hline System 2 & $0.0752-1$ & 0.075 & 2 & 1 & 27-Nov & 73 & 1.7 & 1.73 & 1.71 & 1.69 \\
\hline System 2 & $0.0752-1$ & 0.075 & 2 & 1 & 3-Dec & 73 & 1.7 & 1.72 & 1.7 & 1.69 \\
\hline System 2 & $0.0752-1$ & 0.075 & 2 & 1 & 4-Dec & 70 & 1.7 & 1.71 & 1.7 & 1.69 \\
\hline System 2 & $0.0752-2$ & 0.075 & 2 & 2 & 19-Nov & 73 & 1.7 & 1.72 & 1.69 & 1.7 \\
\hline System 2 & $0.0752-2$ & 0.075 & 2 & 2 & 20-Nov & 63 & 1.72 & 1.73 & 1.71 & 1.72 \\
\hline System 2 & $0.0752-2$ & 0.075 & 2 & 2 & 21-Nov & 70 & 1.73 & 1.73 & 1.71 & 1.72 \\
\hline System 2 & $0.0752-2$ & 0.075 & 2 & 2 & 26-Nov & 70 & 1.74 & 1.73 & 1.72 & 1.73 \\
\hline System 2 & $0.0752-2$ & 0.075 & 2 & 2 & 27-Nov & 73 & 1.73 & 1.73 & 1.72 & 1.72 \\
\hline System 2 & $0.0752-2$ & 0.075 & 2 & 2 & 3-Dec & 73 & 1.74 & 1.73 & 1.72 & 1.72 \\
\hline System 2 & $0.0752-2$ & 0.075 & 2 & 2 & 4-Dec & 70 & 1.72 & 1.72 & 1.71 & 1.71 \\
\hline System 2 & $0.0752-3$ & 0.075 & 2 & 3 & 19-Nov & 73 & 1.69 & 1.69 & 1.71 & 1.69 \\
\hline System 2 & $0.0752-3$ & 0.075 & 2 & 3 & 20-Nov & 63 & 1.71 & 1.71 & 1.71 & 1.71 \\
\hline System 2 & $0.0752-3$ & 0.075 & 2 & 3 & 21-Nov & 70 & 1.71 & 1.72 & 1.72 & 1.71 \\
\hline System 2 & $0.0752-3$ & 0.075 & 2 & 3 & 26-Nov & 70 & 1.71 & 1.73 & 1.74 & 1.73 \\
\hline System 2 & $0.0752-3$ & 0.075 & 2 & 3 & 27-Nov & 73 & 1.71 & 1.73 & 1.71 & 1.72 \\
\hline System 2 & $0.0752-3$ & 0.075 & 2 & 3 & 3-Dec & 73 & 1.73 & 1.72 & 1.71 & 1.71 \\
\hline System 2 & $0.0752-3$ & 0.075 & 2 & 3 & 4-Dec & 70 & 1.7 & 1.72 & 1.71 & 1.7 \\
\hline System 2 & $0.0753-1$ & 0.075 & 3 & 1 & 19-Nov & 73 & 1.69 & 1.68 & 1.7 & 1.69 \\
\hline System 2 & $0.0753-1$ & 0.075 & 3 & 1 & 20-Nov & 63 & 1.7 & 1.7 & 1.71 & 1.71 \\
\hline System 2 & $0.0753-1$ & 0.075 & 3 & 1 & 21-Nov & 70 & 1.7 & 1.69 & 1.71 & 1.71 \\
\hline System 2 & $0.0753-1$ & 0.075 & 3 & 1 & 26-Nov & 70 & 1.71 & 1.71 & 1.73 & 1.72 \\
\hline System 2 & $0.0753-1$ & 0.075 & 3 & 1 & 27-Nov & 73 & 1.71 & 1.71 & 1.73 & 1.72 \\
\hline System 2 & $0.0753-1$ & 0.075 & 3 & 1 & 3-Dec & 73 & 1.71 & 1.71 & 1.71 & 1.72 \\
\hline System 2 & $0.0753-1$ & 0.075 & 3 & 1 & 4-Dec & 70 & 1.7 & 1.7 & 1.71 & 1.71 \\
\hline System 2 & $0.0753-2$ & 0.075 & 3 & 2 & 19-Nov & 73 & 1.67 & 1.69 & 1.7 & 1.7 \\
\hline System 2 & $0.0753-2$ & 0.075 & 3 & 2 & 20-Nov & 63 & 1.7 & 1.71 & 1.73 & 1.72 \\
\hline System 2 & $0.0753-2$ & 0.075 & 3 & 2 & 21-Nov & 70 & 1.71 & 1.71 & 1.73 & 1.73 \\
\hline System 2 & $0.0753-2$ & 0.075 & 3 & 2 & 26-Nov & 70 & 1.72 & 1.73 & 1.74 & 1.75 \\
\hline System 2 & $0.0753-2$ & 0.075 & 3 & 2 & 27-Nov & 73 & 1.7 & 1.7 & 1.73 & 1.72 \\
\hline System 2 & $0.0753-2$ & 0.075 & 3 & 2 & 3-Dec & 73 & 1.71 & 1.71 & 1.74 & 1.73 \\
\hline System 2 & $0.0753-2$ & 0.075 & 3 & 2 & 4-Dec & 70 & 1.71 & 1.7 & 1.73 & 1.73 \\
\hline System 2 & $0.0753-3$ & 0.075 & 3 & 3 & 19-Nov & 73 & 1.67 & 1.68 & 1.68 & 1.68 \\
\hline System 2 & $0.0753-3$ & 0.075 & 3 & 3 & 20-Nov & 63 & 1.7 & 1.7 & 1.69 & 1.7 \\
\hline System 2 & $0.0753-3$ & 0.075 & 3 & 3 & 21-Nov & 70 & 1.7 & 1.7 & 1.7 & 1.7 \\
\hline System 2 & $0.0753-3$ & 0.075 & 3 & 3 & 26-Nov & 70 & 1.71 & 1.72 & 1.71 & 1.71 \\
\hline System 2 & $0.0753-3$ & 0.075 & 3 & 3 & 27-Nov & 73 & 1.71 & 1.71 & 1.71 & 1.71 \\
\hline System 2 & $0.0753-3$ & 0.075 & 3 & 3 & 3-Dec & 73 & 1.71 & 1.71 & 1.72 & 1.72 \\
\hline System 2 & $0.0753-3$ & 0.075 & 3 & 3 & 4-Dec & 70 & 1.7 & 1.7 & 1.71 & 1.7 \\
\hline System 2 & $0.11-1$ & 0.1 & 1 & 1 & 19-Nov & 73 & 2.01 & 2.01 & 2 & 2 \\
\hline System 2 & $0.11-1$ & 0.1 & 1 & 1 & 20-Nov & 63 & 2.02 & 2.02 & 2 & 2.01 \\
\hline System 2 & $0.11-1$ & 0.1 & 1 & 1 & 21-Nov & 70 & 2.02 & 2.03 & 2.01 & 2.01 \\
\hline System 2 & $0.11-1$ & 0.1 & 1 & 1 & 26-Nov & 70 & 2.03 & 2.04 & 2.02 & 2.02 \\
\hline System 2 & $0.11-1$ & 0.1 & 1 & 1 & 27-Nov & 73 & 2.04 & 2.03 & 2.01 & 2.01 \\
\hline System 2 & $0.11-1$ & 0.1 & 1 & 1 & 3-Dec & 73 & 2.03 & 2.03 & 2.02 & 2.01 \\
\hline System 2 & $0.11-1$ & 0.1 & 1 & 1 & 4-Dec & 70 & 2.03 & 2.03 & 2.02 & 2.02 \\
\hline System 2 & $0.11-2$ & 0.1 & 1 & 2 & 19-Nov & 73 & 1.99 & 1.99 & 1.99 & 1.96 \\
\hline System 2 & $0.11-2$ & 0.1 & 1 & 2 & 20-Nov & 63 & 2.01 & 2.01 & 1.99 & 1.96 \\
\hline System 2 & $0.11-2$ & 0.1 & 1 & 2 & 21-Nov & 70 & 2.01 & 2.01 & 2 & 1.96 \\
\hline
\end{tabular}


Table A1. Concentration Unit (CU) Measurements

\begin{tabular}{|c|c|c|c|c|c|c|c|c|c|c|}
\hline \multirow{2}{*}{ Meter } & \multirow{2}{*}{$\begin{array}{l}\text { Peanut } \\
\text { Vial ID }\end{array}$} & \multirow{2}{*}{ Wt\% } & \multirow{2}{*}{ Bottle } & \multirow{2}{*}{ Run } & \multirow{2}{*}{ Date } & \multirow{2}{*}{ Temp } & & pncentratio & Jnits ( & \\
\hline & & & & & & & Back & Forward & Left & Right \\
\hline System 2 & $0.11-2$ & 0.1 & 1 & 2 & 26-Nov & 70 & 2.03 & 2.02 & 2.01 & 1.98 \\
\hline System 2 & $0.11-2$ & 0.1 & 1 & 2 & 27-Nov & 73 & 2.01 & 2 & 1.99 & 1.96 \\
\hline System 2 & $0.11-2$ & 0.1 & 1 & 2 & 3-Dec & 73 & 2.03 & 2.01 & 1.99 & 1.96 \\
\hline System 2 & $0.11-2$ & 0.1 & 1 & 2 & 4-Dec & 70 & 2.02 & 2.01 & 2 & 1.97 \\
\hline System 2 & $0.11-3$ & 0.1 & 1 & 3 & 19-Nov & 73 & 1.96 & 1.97 & 1.98 & 1.97 \\
\hline System 2 & $0.11-3$ & 0.1 & 1 & 3 & 20-Nov & 63 & 1.97 & 1.97 & 1.99 & 1.99 \\
\hline System 2 & $0.11-3$ & 0.1 & 1 & 3 & 21-Nov & 70 & 1.98 & 1.98 & 1.99 & 2 \\
\hline System 2 & $0.11-3$ & 0.1 & 1 & 3 & 26-Nov & 70 & 1.99 & 1.99 & 2 & 2 \\
\hline System 2 & $0.11-3$ & 0.1 & 1 & 3 & 27-Nov & 73 & 1.97 & 1.97 & 1.99 & 1.98 \\
\hline System 2 & $0.11-3$ & 0.1 & 1 & 3 & 3-Dec & 73 & 1.97 & 1.98 & 1.99 & 1.98 \\
\hline System 2 & $0.11-3$ & 0.1 & 1 & 3 & 4-Dec & 70 & 1.99 & 1.98 & 1.99 & 1.99 \\
\hline System 2 & $0.12-1$ & 0.1 & 2 & 1 & 19-Nov & 73 & 1.97 & 1.98 & 1.97 & 1.97 \\
\hline System 2 & $0.12-1$ & 0.1 & 2 & 1 & 20-Nov & 63 & 2 & 2 & 1.98 & 1.98 \\
\hline System 2 & $0.12-1$ & 0.1 & 2 & 1 & 21-Nov & 70 & 2.01 & 2 & 1.99 & 1.99 \\
\hline System 2 & $0.12-1$ & 0.1 & 2 & 1 & 26-Nov & 70 & 2.02 & 2.02 & 2 & 2 \\
\hline System 2 & $0.12-1$ & 0.1 & 2 & 1 & 27-Nov & 73 & 2.01 & 2.01 & 1.98 & 1.99 \\
\hline System 2 & $0.12-1$ & 0.1 & 2 & 1 & 3-Dec & 73 & 2.02 & 2.01 & 2 & 1.99 \\
\hline System 2 & $0.12-1$ & 0.1 & 2 & 1 & 4-Dec & 70 & 2.02 & 2.02 & 2 & 2.01 \\
\hline System 2 & $0.12-2$ & 0.1 & 2 & 2 & 19-Nov & 73 & 1.99 & 2.01 & 2 & 2.01 \\
\hline System 2 & $0.12-2$ & 0.1 & 2 & 2 & 20-Nov & 63 & 2.02 & 2.03 & 2.01 & 2.01 \\
\hline System 2 & $0.12-2$ & 0.1 & 2 & 2 & 21-Nov & 70 & 2.02 & 2.03 & 2.02 & 2.01 \\
\hline System 2 & $0.12-2$ & 0.1 & 2 & 2 & 26-Nov & 70 & 2.02 & 2.04 & 2.03 & 2.03 \\
\hline System 2 & $0.12-2$ & 0.1 & 2 & 2 & 27-Nov & 73 & 2.03 & 2.03 & 2.01 & 2.01 \\
\hline System 2 & $0.12-2$ & 0.1 & 2 & 2 & 3-Dec & 73 & 2.03 & 2.03 & 2.02 & 2.02 \\
\hline System 2 & $0.12-2$ & 0.1 & 2 & 2 & 4-Dec & 70 & 2.02 & 2.02 & 2.02 & 2.01 \\
\hline System 2 & $0.12-3$ & 0.1 & 2 & 3 & 19-Nov & 73 & 2.01 & 2.02 & 2 & 2 \\
\hline System 2 & $0.12-3$ & 0.1 & 2 & 3 & 20-Nov & 63 & 2.03 & 2.02 & 2.01 & 2.01 \\
\hline System 2 & $0.12-3$ & 0.1 & 2 & 3 & 21-Nov & 70 & 2.03 & 2.03 & 2.02 & 2.02 \\
\hline System 2 & $0.12-3$ & 0.1 & 2 & 3 & 26-Nov & 70 & 2.04 & 2.04 & 2.02 & 2.03 \\
\hline System 2 & $0.12-3$ & 0.1 & 2 & 3 & 27-Nov & 73 & 2.02 & 2.02 & 2.01 & 2.02 \\
\hline System 2 & $0.12-3$ & 0.1 & 2 & 3 & 3-Dec & 73 & 2.03 & 2.03 & 2.03 & 2.02 \\
\hline System 2 & $0.12-3$ & 0.1 & 2 & 3 & 4-Dec & 70 & 2.02 & 2.03 & 2 & 2 \\
\hline System 2 & $0.13-1$ & 0.1 & 3 & 1 & 19-Nov & 73 & 1.97 & 1.97 & 1.98 & 1.97 \\
\hline System 2 & $0.13-1$ & 0.1 & 3 & 1 & 20-Nov & 63 & 1.99 & 1.98 & 1.98 & 1.98 \\
\hline System 2 & $0.13-1$ & 0.1 & 3 & 1 & 21-Nov & 70 & 1.99 & 1.98 & 2 & 1.99 \\
\hline System 2 & $0.13-1$ & 0.1 & 3 & 1 & 26-Nov & 70 & 2 & 2 & 2.01 & 2 \\
\hline System 2 & $0.13-1$ & 0.1 & 3 & 1 & 27-Nov & 73 & 1.98 & 1.98 & 1.99 & 1.99 \\
\hline System 2 & $0.13-1$ & 0.1 & 3 & 1 & 3-Dec & 73 & 2 & 1.99 & 2 & 1.98 \\
\hline System 2 & $0.13-1$ & 0.1 & 3 & 1 & 4-Dec & 70 & 1.99 & 1.99 & 1.99 & 1.98 \\
\hline System 2 & $0.13-2$ & 0.1 & 3 & 2 & 19-Nov & 73 & 1.97 & 2 & 1.99 & 2 \\
\hline System 2 & $0.13-2$ & 0.1 & 3 & 2 & 20-Nov & 63 & 2.02 & 2.02 & 2.01 & 1.99 \\
\hline System 2 & $0.13-2$ & 0.1 & 3 & 2 & 21-Nov & 70 & 2.01 & 2.01 & 2.01 & 2.01 \\
\hline System 2 & $0.13-2$ & 0.1 & 3 & 2 & 26-Nov & 70 & 2.02 & 2.03 & 2.02 & 2.02 \\
\hline System 2 & $0.13-2$ & 0.1 & 3 & 2 & 27-Nov & 73 & 2 & 2.01 & 2.01 & 1.98 \\
\hline System 2 & $0.13-2$ & 0.1 & 3 & 2 & 3-Dec & 73 & 2.02 & 2.01 & 2.01 & 2.02 \\
\hline System 2 & $0.13-2$ & 0.1 & 3 & 2 & 4-Dec & 70 & 2 & 2.01 & 2.01 & 1.99 \\
\hline System 2 & $0.13-3$ & 0.1 & 3 & 3 & 19-Nov & 73 & 1.95 & 1.97 & 1.97 & 1.97 \\
\hline System 2 & $0.13-3$ & 0.1 & 3 & 3 & 20-Nov & 63 & 1.97 & 1.97 & 1.99 & 1.98 \\
\hline System 2 & $0.13-3$ & 0.1 & 3 & 3 & 21-Nov & 70 & 1.97 & 1.97 & 1.98 & 1.98 \\
\hline System 2 & $0.13-3$ & 0.1 & 3 & 3 & 26-Nov & 70 & 1.98 & 2 & 2 & 2 \\
\hline System 2 & $0.13-3$ & 0.1 & 3 & 3 & 27-Nov & 73 & 2 & 1.98 & 1.98 & 1.99 \\
\hline System 2 & $0.13-3$ & 0.1 & 3 & 3 & 3-Dec & 73 & 1.99 & 1.98 & 1.99 & 2 \\
\hline System 2 & $0.13-3$ & 0.1 & 3 & 3 & 4-Dec & 70 & 2 & 1.98 & 2 & 2 \\
\hline System 2 & $0.141-1$ & 0.14 & 1 & 1 & 19-Nov & 73 & 2.35 & 2.37 & 2.31 & 2.32 \\
\hline System 2 & $0.141-1$ & 0.14 & 1 & 1 & 20-Nov & 63 & 2.37 & 2.37 & 2.33 & 2.33 \\
\hline System 2 & $0.141-1$ & 0.14 & 1 & 1 & 21-Nov & 70 & 2.37 & 2.37 & 2.33 & 2.33 \\
\hline System 2 & $0.141-1$ & 0.14 & 1 & 1 & 26-Nov & 70 & 2.39 & 2.39 & 2.35 & 2.36 \\
\hline System 2 & $0.141-1$ & 0.14 & 1 & 1 & 27-Nov & 73 & 2.38 & 2.39 & 2.35 & 2.34 \\
\hline System 2 & $0.14 \quad 1-1$ & 0.14 & 1 & 1 & 3-Dec & 73 & 2.39 & 2.38 & 2.33 & 2.35 \\
\hline System 2 & $0.141-1$ & 0.14 & 1 & 1 & 4-Dec & 70 & 2.38 & 2.38 & 2.35 & 2.35 \\
\hline System 2 & $0.141-2$ & 0.14 & 1 & 2 & 19-Nov & 73 & 2.32 & 2.32 & 2.3 & 2.31 \\
\hline System 2 & $0.14 \quad 1-2$ & 0.14 & 1 & 2 & 20-Nov & 63 & 2.34 & 2.33 & 2.31 & 2.32 \\
\hline System 2 & $0.141-2$ & 0.14 & 1 & 2 & 21-Nov & 70 & 2.34 & 2.34 & 2.32 & 2.33 \\
\hline System 2 & $0.141-2$ & 0.14 & 1 & 2 & 26-Nov & 70 & 2.35 & 2.34 & 2.34 & 2.34 \\
\hline System 2 & $0.141-2$ & 0.14 & 1 & 2 & 27-Nov & 73 & 2.34 & 2.33 & 2.33 & 2.33 \\
\hline System 2 & $0.141-2$ & 0.14 & 1 & 2 & 3-Dec & 73 & 2.35 & 2.34 & 2.33 & 2.34 \\
\hline System 2 & $0.141-2$ & 0.14 & 1 & 2 & 4-Dec & 70 & 2.33 & 2.34 & 2.33 & 2.33 \\
\hline System 2 & $0.141-3$ & 0.14 & 1 & 3 & 19-Nov & 73 & 2.32 & 2.3 & 2.29 & 2.31 \\
\hline System 2 & $0.141-3$ & 0.14 & 1 & 3 & 20-Nov & 63 & 2.35 & 2.34 & 2.31 & 2.32 \\
\hline System 2 & $0.141-3$ & 0.14 & 1 & 3 & 21-Nov & 70 & 2.35 & 2.35 & 2.31 & 2.32 \\
\hline System 2 & $0.141-3$ & 0.14 & 1 & 3 & 26-Nov & 70 & 2.36 & 2.37 & 2.32 & 2.34 \\
\hline
\end{tabular}


Table A1. Concentration Unit (CU) Measurements

\begin{tabular}{|c|c|c|c|c|c|c|c|c|c|c|}
\hline \multirow{2}{*}{ Meter } & \multirow{2}{*}{$\begin{array}{l}\text { Peanut } \\
\text { Vial ID } \\
\end{array}$} & \multirow{2}{*}{ Wt\% } & \multirow{2}{*}{ Bottle } & \multirow{2}{*}{ Run } & \multirow{2}{*}{ Date } & \multirow{2}{*}{ Temp } & & oncentratio & Jnits ( & \\
\hline & & & & & & & Back & Forward & Left & Right \\
\hline System 2 & $0.141-3$ & 0.14 & 1 & 3 & 27-Nov & 73 & 2.35 & 2.36 & 2.32 & 2.33 \\
\hline System 2 & $0.141-3$ & 0.14 & 1 & 3 & 3-Dec & 73 & 2.37 & 2.35 & 2.33 & 2.33 \\
\hline System 2 & $0.141-3$ & 0.14 & 1 & 3 & 4-Dec & 70 & 2.37 & 2.37 & 2.33 & 2.34 \\
\hline System 2 & $0.142-1$ & 0.14 & 2 & 1 & 19-Nov & 73 & 2.31 & 2.31 & 2.33 & 2.32 \\
\hline System 2 & $0.142-1$ & 0.14 & 2 & 1 & 20-Nov & 63 & 2.33 & 2.33 & 2.35 & 2.34 \\
\hline System 2 & $0.142-1$ & 0.14 & 2 & 1 & 21-Nov & 70 & 2.34 & 2.34 & 2.35 & 2.35 \\
\hline System 2 & $0.142-1$ & 0.14 & 2 & 1 & 26-Nov & 70 & 2.34 & 2.34 & 2.36 & 2.36 \\
\hline System 2 & $0.142-1$ & 0.14 & 2 & 1 & 27-Nov & 73 & 2.35 & 2.35 & 2.36 & 2.34 \\
\hline System 2 & $0.142-1$ & 0.14 & 2 & 1 & 3-Dec & 73 & 2.34 & 2.35 & 2.36 & 2.35 \\
\hline System 2 & $0.142-1$ & 0.14 & 2 & 1 & 4-Dec & 70 & 2.35 & 2.36 & 2.33 & 2.34 \\
\hline System 2 & $0.142-2$ & 0.14 & 2 & 2 & 19-Nov & 73 & 2.31 & 2.32 & 2.32 & 2.31 \\
\hline System 2 & $0.142-2$ & 0.14 & 2 & 2 & 20-Nov & 63 & 2.34 & 2.34 & 2.31 & 2.32 \\
\hline System 2 & $0.142-2$ & 0.14 & 2 & 2 & 21-Nov & 70 & 2.34 & 2.33 & 2.33 & 2.32 \\
\hline System 2 & $0.142-2$ & 0.14 & 2 & 2 & 26-Nov & 70 & 2.35 & 2.35 & 2.32 & 2.32 \\
\hline System 2 & $0.142-2$ & 0.14 & 2 & 2 & 27-Nov & 73 & 2.33 & 2.32 & 2.31 & 2.32 \\
\hline System 2 & $0.142-2$ & 0.14 & 2 & 2 & 3-Dec & 73 & 2.34 & 2.33 & 2.3 & 2.32 \\
\hline System 2 & $0.142-2$ & 0.14 & 2 & 2 & 4-Dec & 70 & 2.34 & 2.34 & 2.32 & 2.32 \\
\hline System 2 & $0.142-3$ & 0.14 & 2 & 3 & 19-Nov & 73 & 2.35 & 2.34 & 2.33 & 2.34 \\
\hline System 2 & $0.142-3$ & 0.14 & 2 & 3 & 20-Nov & 63 & 2.36 & 2.36 & 2.35 & 2.34 \\
\hline System 2 & $0.142-3$ & 0.14 & 2 & 3 & 21-Nov & 70 & 2.37 & 2.37 & 2.36 & 2.35 \\
\hline System 2 & $0.142-3$ & 0.14 & 2 & 3 & 26-Nov & 70 & 2.38 & 2.37 & 2.37 & 2.35 \\
\hline System 2 & $0.142-3$ & 0.14 & 2 & 3 & 27-Nov & 73 & 2.35 & 2.36 & 2.32 & 2.34 \\
\hline System 2 & $0.142-3$ & 0.14 & 2 & 3 & 3-Dec & 73 & 2.38 & 2.37 & 2.37 & 2.38 \\
\hline System 2 & $0.142-3$ & 0.14 & 2 & 3 & 4-Dec & 70 & 2.38 & 2.37 & 2.37 & 2.37 \\
\hline System 2 & $0.143-1$ & 0.14 & 3 & 1 & 19-Nov & 73 & 2.33 & 2.33 & 2.35 & 2.35 \\
\hline System 2 & $0.143-1$ & 0.14 & 3 & 1 & 20-Nov & 63 & 2.35 & 2.35 & 2.37 & 2.37 \\
\hline System 2 & $0.143-1$ & 0.14 & 3 & 1 & 21-Nov & 70 & 2.36 & 2.35 & 2.37 & 2.36 \\
\hline System 2 & $0.143-1$ & 0.14 & 3 & 1 & 26-Nov & 70 & 2.36 & 2.37 & 2.39 & 2.38 \\
\hline System 2 & $0.143-1$ & 0.14 & 3 & 1 & 27-Nov & 73 & 2.35 & 2.36 & 2.36 & 2.37 \\
\hline System 2 & $0.143-1$ & 0.14 & 3 & 1 & 3-Dec & 73 & 2.36 & 2.35 & 2.37 & 2.38 \\
\hline System 2 & $0.143-1$ & 0.14 & 3 & 1 & 4-Dec & 70 & 2.36 & 2.35 & 2.37 & 2.37 \\
\hline System 2 & $0.143-2$ & 0.14 & 3 & 2 & 19-Nov & 73 & 2.36 & 2.36 & 2.35 & 2.35 \\
\hline System 2 & $0.143-2$ & 0.14 & 3 & 2 & 20-Nov & 63 & 2.38 & 2.37 & 2.37 & 2.37 \\
\hline System 2 & $0.143-2$ & 0.14 & 3 & 2 & 21-Nov & 70 & 2.38 & 2.37 & 2.37 & 2.37 \\
\hline System 2 & $0.143-2$ & 0.14 & 3 & 2 & 26-Nov & 70 & 2.39 & 2.38 & 2.38 & 2.38 \\
\hline System 2 & $0.143-2$ & 0.14 & 3 & 2 & 27-Nov & 73 & 2.39 & 2.39 & 2.38 & 2.38 \\
\hline System 2 & $0.143-2$ & 0.14 & 3 & 2 & 3-Dec & 73 & 2.38 & 2.38 & 2.38 & 2.37 \\
\hline System 2 & $0.143-2$ & 0.14 & 3 & 2 & 4-Dec & 70 & 2.38 & 2.38 & 2.38 & 2.38 \\
\hline System 2 & $0.143-3$ & 0.14 & 3 & 3 & 19-Nov & 73 & 2.34 & 2.35 & 2.35 & 2.35 \\
\hline System 2 & $0.143-3$ & 0.14 & 3 & 3 & 20-Nov & 63 & 2.36 & 2.36 & 2.36 & 2.36 \\
\hline System 2 & $0.143-3$ & 0.14 & 3 & 3 & 21-Nov & 70 & 2.37 & 2.37 & 2.35 & 2.36 \\
\hline System 2 & $0.143-3$ & 0.14 & 3 & 3 & 26-Nov & 70 & 2.37 & 2.38 & 2.37 & 2.36 \\
\hline System 2 & $0.143-3$ & 0.14 & 3 & 3 & 27-Nov & 73 & 2.37 & 2.37 & 2.37 & 2.36 \\
\hline System 2 & $0.143-3$ & 0.14 & 3 & 3 & 3-Dec & 73 & 2.37 & 2.37 & 2.37 & 2.37 \\
\hline System 2 & $0.143-3$ & 0.14 & 3 & 3 & 4-Dec & 70 & 2.37 & 2.37 & 2.36 & 2.37 \\
\hline System 2 & 0.15 & 0.15 & 1 & 1 & 19-Nov & 73 & 2.41 & 2.4 & 2.43 & 2.41 \\
\hline System 2 & 0.15 & 0.15 & 1 & 1 & 20-Nov & 63 & 2.42 & 2.42 & 2.43 & 2.42 \\
\hline System 2 & 0.15 & 0.15 & 1 & 1 & 21-Nov & 70 & 2.43 & 2.43 & 2.43 & 2.43 \\
\hline System 2 & 0.15 & 0.15 & 1 & 1 & 26-Nov & 70 & 2.43 & 2.44 & 2.44 & 2.43 \\
\hline System 2 & 0.15 & 0.15 & 1 & 1 & 27-Nov & 73 & 2.43 & 2.44 & 2.45 & 2.43 \\
\hline System 2 & 0.15 & 0.15 & 1 & 1 & 3-Dec & 73 & 2.45 & 2.44 & 2.45 & 2.43 \\
\hline System 2 & 0.15 & 0.15 & 1 & 1 & 4-Dec & 70 & 2.44 & 2.44 & 2.46 & 2.43 \\
\hline System 2 & $0.21-1$ & 0.2 & 1 & 1 & 19-Nov & 73 & 2.69 & 2.7 & 2.7 & 2.71 \\
\hline System 2 & $0.21-1$ & 0.2 & 1 & 1 & 20-Nov & 63 & 2.7 & 2.7 & 2.71 & 2.71 \\
\hline System 2 & $0.21-1$ & 0.2 & 1 & 1 & 21-Nov & 70 & 2.69 & 2.7 & 2.71 & 2.71 \\
\hline System 2 & $0.21-1$ & 0.2 & 1 & 1 & 26-Nov & 70 & 2.69 & 2.71 & 2.72 & 2.73 \\
\hline System 2 & $0.21-1$ & 0.2 & 1 & 1 & 27-Nov & 73 & 2.71 & 2.71 & 2.72 & 2.72 \\
\hline System 2 & $0.21-1$ & 0.2 & 1 & 1 & 3-Dec & 73 & 2.71 & 2.71 & 2.72 & 2.72 \\
\hline System 2 & $0.21-1$ & 0.2 & 1 & 1 & 4-Dec & 70 & 2.71 & 2.72 & 2.73 & 2.73 \\
\hline System 2 & $0.21-2$ & 0.2 & 1 & 2 & 19-Nov & 73 & 2.68 & 2.67 & 2.67 & 2.66 \\
\hline System 2 & $0.21-2$ & 0.2 & 1 & 2 & 20-Nov & 63 & 2.69 & 2.68 & 2.68 & 2.67 \\
\hline System 2 & $0.21-2$ & 0.2 & 1 & 2 & 21-Nov & 70 & 2.69 & 2.68 & 2.68 & 2.67 \\
\hline System 2 & $0.21-2$ & 0.2 & 1 & 2 & 26-Nov & 70 & 2.69 & 2.69 & 2.69 & 2.68 \\
\hline System 2 & $0.21-2$ & 0.2 & 1 & 2 & 27-Nov & 73 & 2.7 & 2.7 & 2.7 & 2.69 \\
\hline System 2 & $0.21-2$ & 0.2 & 1 & 2 & 3-Dec & 73 & 2.71 & 2.7 & 2.69 & 2.69 \\
\hline System 2 & $0.21-2$ & 0.2 & 1 & 2 & 4-Dec & 70 & 2.69 & 2.7 & 2.7 & 2.69 \\
\hline System 2 & $0.21-3$ & 0.2 & 1 & 3 & 19-Nov & 73 & 2.69 & 2.69 & 2.68 & 2.68 \\
\hline System 2 & $0.21-3$ & 0.2 & 1 & 3 & 20-Nov & 63 & 2.7 & 2.7 & 2.69 & 2.69 \\
\hline System 2 & $0.21-3$ & 0.2 & 1 & 3 & 21-Nov & 70 & 2.71 & 2.71 & 2.69 & 2.7 \\
\hline System 2 & $0.21-3$ & 0.2 & 1 & 3 & 26-Nov & 70 & 2.71 & 2.71 & 2.7 & 2.71 \\
\hline System 2 & $0.21-3$ & 0.2 & 1 & 3 & 27-Nov & 73 & 2.72 & 2.73 & 2.7 & 2.7 \\
\hline
\end{tabular}


Table A1. Concentration Unit (CU) Measurements

\begin{tabular}{|c|c|c|c|c|c|c|c|c|c|c|}
\hline \multirow{2}{*}{ Meter } & \multirow{2}{*}{$\begin{array}{l}\text { Peanut } \\
\text { Vial ID }\end{array}$} & \multirow{2}{*}{ Wt\% } & \multirow{2}{*}{ Bottle } & \multirow{2}{*}{ Run } & \multirow{2}{*}{ Date } & \multirow{2}{*}{ Temp } & & ncentratio & Jnits ( & \\
\hline & & & & & & & Back & Forward & Left & Right \\
\hline System 2 & $0.21-3$ & 0.2 & 1 & 3 & 3-Dec & 73 & 2.73 & 2.74 & 2.71 & 2.71 \\
\hline System 2 & $0.21-3$ & 0.2 & 1 & 3 & 4-Dec & 70 & 2.73 & 2.73 & 2.71 & 2.71 \\
\hline System 2 & $0.22-1$ & 0.2 & 2 & 1 & 19-Nov & 73 & 2.69 & 2.7 & 2.7 & 2.69 \\
\hline System 2 & $0.22-1$ & 0.2 & 2 & 1 & 20-Nov & 63 & 2.71 & 2.71 & 2.7 & 2.71 \\
\hline System 2 & $0.22-1$ & 0.2 & 2 & 1 & 21-Nov & 70 & 2.71 & 2.7 & 2.71 & 2.7 \\
\hline System 2 & $0.22-1$ & 0.2 & 2 & 1 & 26-Nov & 70 & 2.72 & 2.72 & 2.73 & 2.72 \\
\hline System 2 & $0.22-1$ & 0.2 & 2 & 1 & 27-Nov & 73 & 2.72 & 2.73 & 2.72 & 2.73 \\
\hline System 2 & $0.22-1$ & 0.2 & 2 & 1 & 3-Dec & 73 & 2.73 & 2.73 & 2.73 & 2.73 \\
\hline System 2 & $0.22-1$ & 0.2 & 2 & 1 & 4-Dec & 70 & 2.72 & 2.73 & 2.73 & 2.73 \\
\hline System 2 & $0.22-2$ & 0.2 & 2 & 2 & 19-Nov & 73 & 2.7 & 2.72 & 2.7 & 2.69 \\
\hline System 2 & $0.22-2$ & 0.2 & 2 & 2 & 20-Nov & 63 & 2.72 & 2.72 & 2.7 & 2.7 \\
\hline System 2 & $0.22-2$ & 0.2 & 2 & 2 & 21-Nov & 70 & 2.72 & 2.72 & 2.71 & 2.7 \\
\hline System 2 & $0.22-2$ & 0.2 & 2 & 2 & 26-Nov & 70 & 2.73 & 2.72 & 2.72 & 2.72 \\
\hline System 2 & $0.22-2$ & 0.2 & 2 & 2 & 27-Nov & 73 & 2.74 & 2.73 & 2.72 & 2.72 \\
\hline System 2 & $0.22-2$ & 0.2 & 2 & 2 & 3-Dec & 73 & 2.75 & 2.75 & 2.73 & 2.74 \\
\hline System 2 & $0.22-2$ & 0.2 & 2 & 2 & 4-Dec & 70 & 2.74 & 2.75 & 2.74 & 2.73 \\
\hline System 2 & $0.22-3$ & 0.2 & 2 & 3 & 19-Nov & 73 & 2.69 & 2.7 & 2.73 & 2.72 \\
\hline System 2 & $0.22-3$ & 0.2 & 2 & 3 & 20-Nov & 63 & 2.71 & 2.7 & 2.74 & 2.73 \\
\hline System 2 & $0.22-3$ & 0.2 & 2 & 3 & 21-Nov & 70 & 2.71 & 2.71 & 2.74 & 2.73 \\
\hline System 2 & $0.22-3$ & 0.2 & 2 & 3 & 26-Nov & 70 & 2.72 & 2.72 & 2.74 & 2.73 \\
\hline System 2 & $0.22-3$ & 0.2 & 2 & 3 & 27-Nov & 73 & 2.71 & 2.72 & 2.75 & 2.74 \\
\hline System 2 & $0.22-3$ & 0.2 & 2 & 3 & 3-Dec & 73 & 2.72 & 2.73 & 2.74 & 2.75 \\
\hline System 2 & $0.22-3$ & 0.2 & 2 & 3 & 4-Dec & 70 & 2.72 & 2.72 & 2.74 & 2.75 \\
\hline System 2 & $0.23-1$ & 0.2 & 3 & 1 & 19-Nov & 73 & 2.68 & 2.69 & 2.69 & 2.7 \\
\hline System 2 & $0.23-1$ & 0.2 & 3 & 1 & 20-Nov & 63 & 2.7 & 2.69 & 2.7 & 2.7 \\
\hline System 2 & $0.23-1$ & 0.2 & 3 & 1 & 21-Nov & 70 & 2.7 & 2.7 & 2.71 & 2.71 \\
\hline System 2 & $0.23-1$ & 0.2 & 3 & 1 & 26-Nov & 70 & 2.71 & 2.72 & 2.72 & 2.72 \\
\hline System 2 & $0.23-1$ & 0.2 & 3 & 1 & 27-Nov & 73 & 2.72 & 2.72 & 2.72 & 2.72 \\
\hline System 2 & $0.23-1$ & 0.2 & 3 & 1 & 3-Dec & 73 & 2.73 & 2.73 & 2.74 & 2.73 \\
\hline System 2 & $0.23-1$ & 0.2 & 3 & 1 & 4-Dec & 70 & 2.73 & 2.73 & 2.74 & 2.73 \\
\hline System 2 & $0.23-2$ & 0.2 & 3 & 2 & 19-Nov & 73 & 2.69 & 2.68 & 2.66 & 2.67 \\
\hline System 2 & $0.23-2$ & 0.2 & 3 & 2 & 20-Nov & 63 & 2.7 & 2.69 & 2.67 & 2.68 \\
\hline System 2 & $0.23-2$ & 0.2 & 3 & 2 & 21-Nov & 70 & 2.7 & 2.69 & 2.68 & 2.67 \\
\hline System 2 & $0.23-2$ & 0.2 & 3 & 2 & 26-Nov & 70 & 2.71 & 2.69 & 2.69 & 2.68 \\
\hline System 2 & $0.23-2$ & 0.2 & 3 & 2 & 27-Nov & 73 & 2.7 & 2.7 & 2.68 & 2.68 \\
\hline System 2 & $0.23-2$ & 0.2 & 3 & 2 & 3-Dec & 73 & 2.71 & 2.71 & 2.69 & 2.69 \\
\hline System 2 & $0.23-2$ & 0.2 & 3 & 2 & 4-Dec & 70 & 2.71 & 2.71 & 2.69 & 2.69 \\
\hline System 2 & $0.23-3$ & 0.2 & 3 & 3 & 19-Nov & 73 & 2.64 & 2.65 & 2.67 & 2.67 \\
\hline System 2 & $0.23-3$ & 0.2 & 3 & 3 & 20-Nov & 63 & 2.67 & 2.66 & 2.68 & 2.68 \\
\hline System 2 & $0.23-3$ & 0.2 & 3 & 3 & 21-Nov & 70 & 2.66 & 2.66 & 2.68 & 2.68 \\
\hline System 2 & $0.23-3$ & 0.2 & 3 & 3 & 26-Nov & 70 & 2.66 & 2.67 & 2.69 & 2.69 \\
\hline System 2 & $0.23-3$ & 0.2 & 3 & 3 & 27-Nov & 73 & 2.67 & 2.67 & 2.68 & 2.7 \\
\hline System 2 & $0.23-3$ & 0.2 & 3 & 3 & 3-Dec & 73 & 2.67 & 2.67 & 2.7 & 2.7 \\
\hline System 2 & $0.23-3$ & 0.2 & 3 & 3 & 4-Dec & 70 & 2.68 & 2.68 & 2.69 & 2.7 \\
\hline System 2 & 0.3 & 0.3 & 1 & 1 & 19-Nov & 73 & 3.1 & 3.1 & 3.11 & 3.12 \\
\hline System 2 & 0.3 & 0.3 & 1 & 1 & 20-Nov & 63 & 3.11 & 3.11 & 3.12 & 3.13 \\
\hline System 2 & 0.3 & 0.3 & 1 & 1 & 21-Nov & 70 & 3.11 & 3.11 & 3.13 & 3.13 \\
\hline System 2 & 0.3 & 0.3 & 1 & 1 & 26-Nov & 70 & 3.12 & 3.13 & 3.14 & 3.14 \\
\hline System 2 & 0.3 & 0.3 & 1 & 1 & 27-Nov & 73 & 3.14 & 3.13 & 3.14 & 3.15 \\
\hline System 2 & 0.3 & 0.3 & 1 & 1 & 3-Dec & 73 & 3.14 & 3.14 & 3.15 & 3.16 \\
\hline System 2 & 0.3 & 0.3 & 1 & 1 & 4-Dec & 70 & 3.14 & 3.14 & 3.15 & 3.16 \\
\hline System 2 & 0.4 & 0.4 & 1 & 1 & 19-Nov & 73 & 3.41 & 3.42 & 3.44 & 3.44 \\
\hline System 2 & 0.4 & 0.4 & 1 & 1 & 20-Nov & 63 & 3.43 & 3.43 & 3.46 & 3.45 \\
\hline System 2 & 0.4 & 0.4 & 1 & 1 & 21-Nov & 70 & 3.42 & 3.43 & 3.45 & 3.45 \\
\hline System 2 & 0.4 & 0.4 & 1 & 1 & 26-Nov & 70 & 3.43 & 3.43 & 3.46 & 3.45 \\
\hline System 2 & 0.4 & 0.4 & 1 & 1 & 27-Nov & 73 & 3.44 & 3.43 & 3.46 & 3.46 \\
\hline System 2 & 0.4 & 0.4 & 1 & 1 & 3-Dec & 73 & 3.45 & 3.44 & 3.47 & 3.47 \\
\hline System 2 & 0.4 & 0.4 & 1 & 1 & 4-Dec & 70 & 3.45 & 3.44 & 3.47 & 3.47 \\
\hline System 2 & 0.5 & 0.5 & 1 & 1 & 19-Nov & 73 & 3.69 & 3.7 & 3.68 & 3.68 \\
\hline System 2 & 0.5 & 0.5 & 1 & 1 & 20-Nov & 63 & 3.7 & 3.71 & 3.7 & 3.69 \\
\hline System 2 & 0.5 & 0.5 & 1 & 1 & 21-Nov & 70 & 3.71 & 3.72 & 3.71 & 3.7 \\
\hline System 2 & 0.5 & 0.5 & 1 & 1 & 26-Nov & 70 & 3.72 & 3.73 & 3.72 & 3.71 \\
\hline System 2 & 0.5 & 0.5 & 1 & 1 & 27-Nov & 73 & 3.74 & 3.74 & 3.73 & 3.72 \\
\hline System 2 & 0.5 & 0.5 & 1 & 1 & 3-Dec & 73 & 3.75 & 3.75 & 3.74 & 3.73 \\
\hline System 2 & 0.5 & 0.5 & 1 & 1 & 4-Dec & 70 & 3.75 & 3.75 & 3.74 & 3.73 \\
\hline System 2 & 0.6 & 0.6 & 1 & 1 & 19-Nov & 73 & 3.95 & 3.95 & 3.95 & 3.94 \\
\hline System 2 & 0.6 & 0.6 & 1 & 1 & 20-Nov & 63 & 3.96 & 3.96 & 3.96 & 3.95 \\
\hline System 2 & 0.6 & 0.6 & 1 & 1 & 21-Nov & 70 & 3.96 & 3.96 & 3.97 & 3.96 \\
\hline System 2 & 0.6 & 0.6 & 1 & 1 & 26-Nov & 70 & 3.97 & 3.98 & 3.99 & 3.97 \\
\hline System 2 & 0.6 & 0.6 & 1 & 1 & 27-Nov & 73 & 4 & 4 & 3.98 & 3.99 \\
\hline System 2 & 0.6 & 0.6 & 1 & 1 & 3-Dec & 73 & 4.01 & 4.01 & 4.01 & 4 \\
\hline
\end{tabular}


SRNL-STI-2013-00016

Revision 0

Table A1. Concentration Unit (CU) Measurements

\begin{tabular}{|c|c|c|c|c|c|c|c|c|c|c|}
\hline \multirow{2}{*}{ Meter } & \multirow{2}{*}{$\begin{array}{l}\text { Peanut } \\
\text { Vial ID }\end{array}$} & \multirow{2}{*}{ Wt\% } & \multirow{2}{*}{ Bottle } & \multirow{2}{*}{ Run } & \multirow{2}{*}{ Date } & \multirow{2}{*}{ Temp } & \multicolumn{4}{|c|}{ Concentration Units (CUs) } \\
\hline & & & & & & & Back & Forward & Left & Right \\
\hline System 2 & 0.6 & 0.6 & 1 & 1 & 4-Dec & 70 & 4.01 & 3.98 & 4.01 & 3.98 \\
\hline System 2 & 0.7 & 0.7 & 1 & 1 & 19-Nov & 73 & 4.11 & 4.16 & 4.14 & 4.15 \\
\hline System 2 & 0.7 & 0.7 & 1 & 1 & 20-Nov & 63 & 4.13 & 4.18 & 4.15 & 4.16 \\
\hline System 2 & 0.7 & 0.7 & 1 & 1 & 21-Nov & 70 & 4.13 & 4.17 & 4.15 & 4.16 \\
\hline System 2 & 0.7 & 0.7 & 1 & 1 & 26-Nov & 70 & 4.14 & 4.18 & 4.16 & 4.17 \\
\hline System 2 & 0.7 & 0.7 & 1 & 1 & 27-Nov & 73 & 4.16 & 4.22 & 4.2 & 4.2 \\
\hline System 2 & 0.7 & 0.7 & 1 & 1 & 3-Dec & 73 & 4.17 & 4.22 & 4.21 & 4.21 \\
\hline System 2 & 0.7 & 0.7 & 1 & 1 & 4-Dec & 70 & 4.17 & 4.22 & 4.21 & 4.21 \\
\hline System 2 & 0.8 & 0.8 & 1 & 1 & 19-Nov & 73 & 4.34 & 4.35 & 4.34 & 4.36 \\
\hline System 2 & 0.8 & 0.8 & 1 & 1 & 20-Nov & 63 & 4.35 & 4.35 & 4.35 & 4.38 \\
\hline System 2 & 0.8 & 0.8 & 1 & 1 & 21-Nov & 70 & 4.36 & 4.37 & 4.35 & 4.38 \\
\hline System 2 & 0.8 & 0.8 & 1 & 1 & 26-Nov & 70 & 4.37 & 4.37 & 4.35 & 4.37 \\
\hline System 2 & 0.8 & 0.8 & 1 & 1 & 27-Nov & 73 & 4.39 & 4.4 & 4.39 & 4.41 \\
\hline System 2 & 0.8 & 0.8 & 1 & 1 & 3-Dec & 73 & 4.41 & 4.41 & 4.4 & 4.43 \\
\hline System 2 & 0.8 & 0.8 & 1 & 1 & 4-Dec & 70 & 4.41 & 4.4 & 4.38 & 4.42 \\
\hline System 2 & 1.2 & 1.2 & 1 & 1 & 19-Nov & 73 & 4.7 & 4.73 & 4.68 & 4.75 \\
\hline System 2 & 1.2 & 1.2 & 1 & 1 & 20-Nov & 63 & 4.72 & 4.75 & 4.71 & 4.77 \\
\hline System 2 & 1.2 & 1.2 & 1 & 1 & 21-Nov & 70 & 4.73 & 4.75 & 4.7 & 4.8 \\
\hline System 2 & 1.2 & 1.2 & 1 & 1 & 26-Nov & 70 & 4.73 & 4.76 & 4.71 & 4.78 \\
\hline System 2 & 1.2 & 1.2 & 1 & 1 & 27-Nov & 73 & 4.76 & 4.78 & 4.75 & 4.82 \\
\hline System 2 & 1.2 & 1.2 & 1 & 1 & 3-Dec & 73 & 4.77 & 4.78 & 4.75 & 4.81 \\
\hline System 2 & 1.2 & 1.2 & 1 & 1 & 4-Dec & 70 & 4.76 & 4.78 & 4.75 & 4.8 \\
\hline
\end{tabular}


Exhibit A1. Concentration Unit (CU) Measurements by Meter for Deionized Water Standards

$\circ$ - forward, $\bullet$ - backward, $\diamond$ - left, and $\bullet$ - right

Meter=System 1: Variability Chart for CU

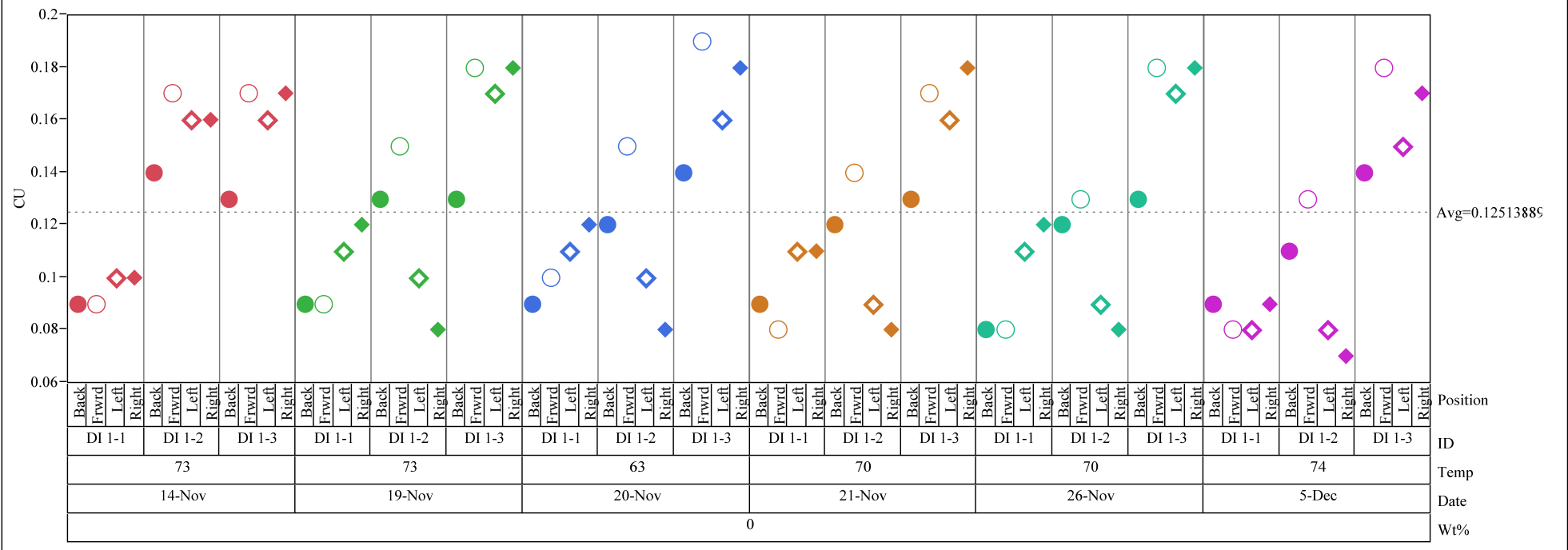

Meter=System 2: Variability Chart for CU

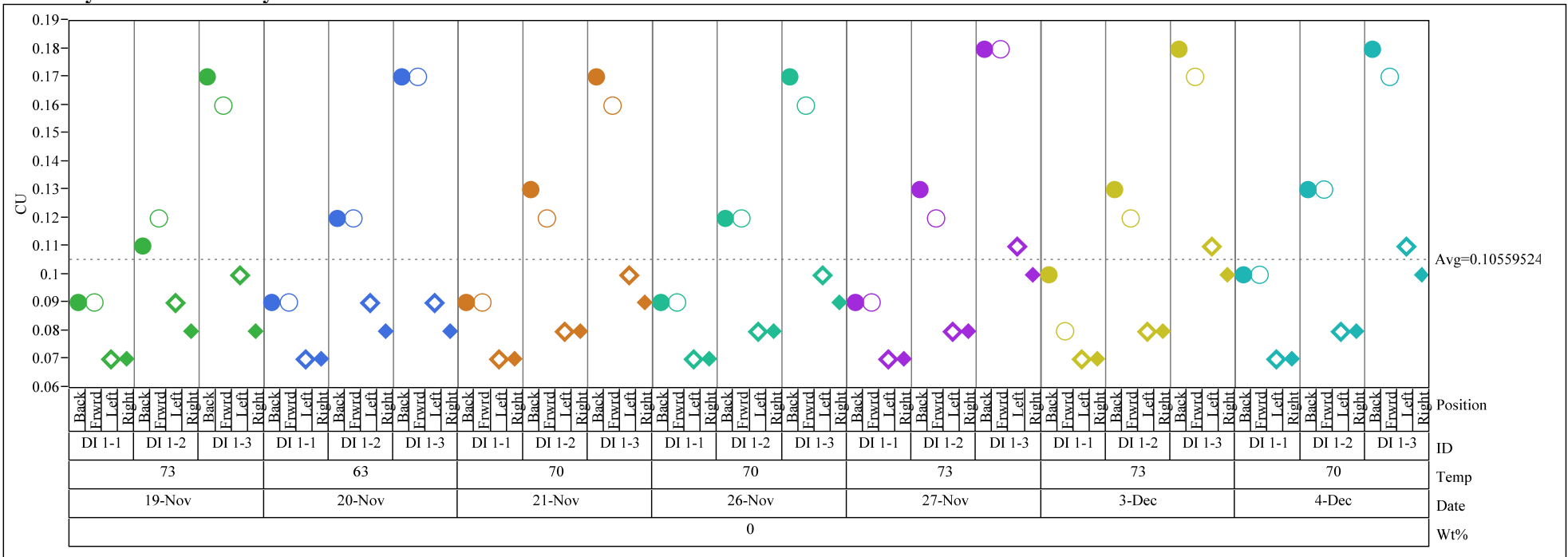


SRNL-STI-2013-00016

Revision 0

Exhibit A2. Concentration Unit (CU) Measurements by Meter for Deionized Water and Sludge Simulant Standards

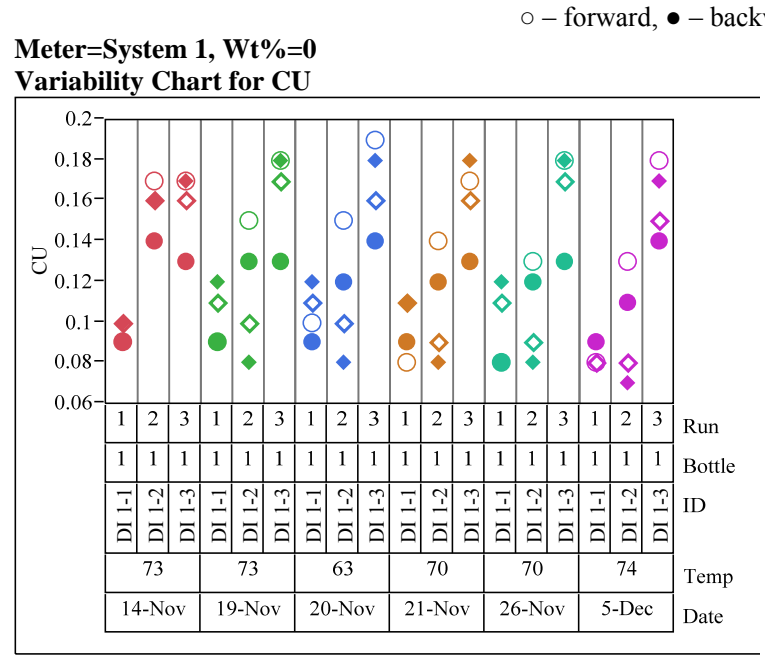

Meter=System 1, Wt $\%=0.05$

Variability Chart for CU

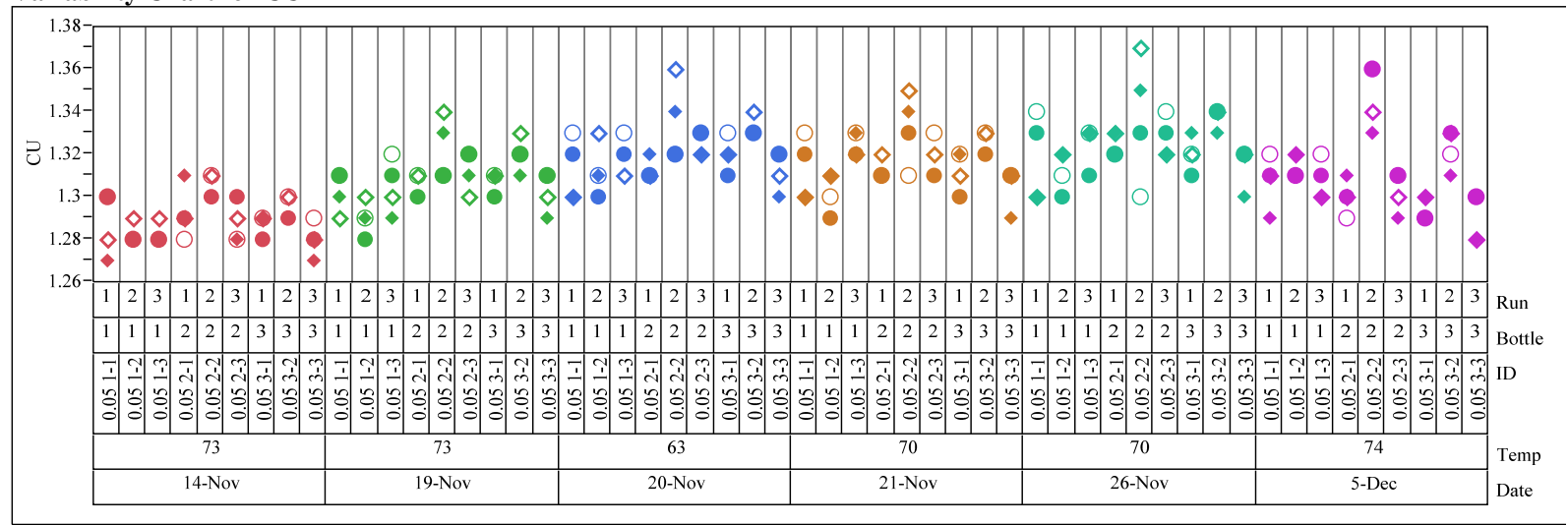

Meter=System 1, Wt \%=0.075

Variability Chart for CU

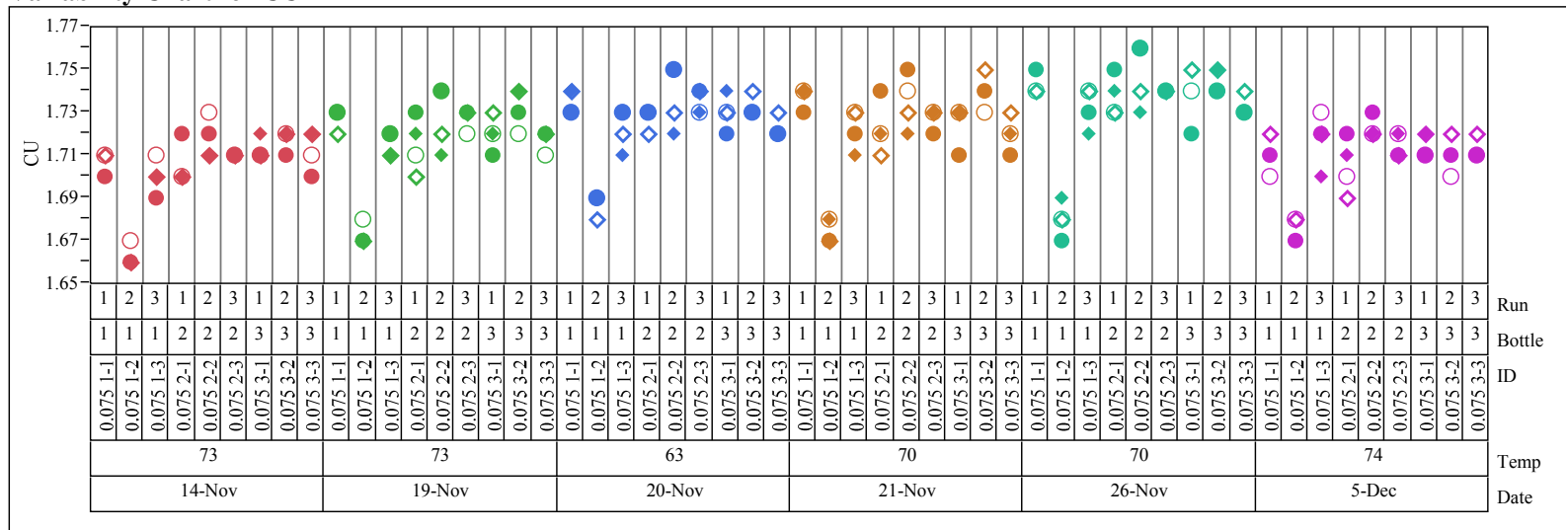


SRNL-STI-2013-00016

Revision 0

Exhibit A2. Concentration Unit (CU) Measurements by Meter for Deionized Water and Sludge Simulant Standards

Meter=System 1, Wt $\%=0.1$

$\circ$ - forward, $\bullet$ - backward, $\diamond-$ left, and $\bullet-$ right

Variability Chart for CU

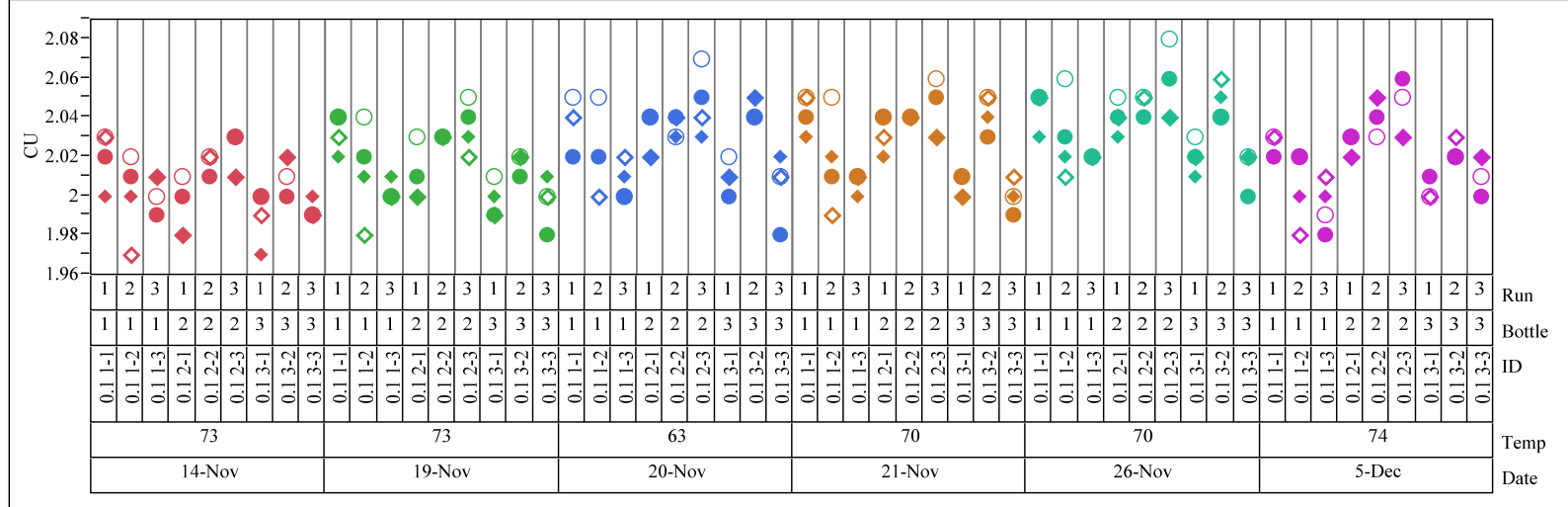

Meter=System 1, Wt $\%=0.14$

Variability Chart for CU

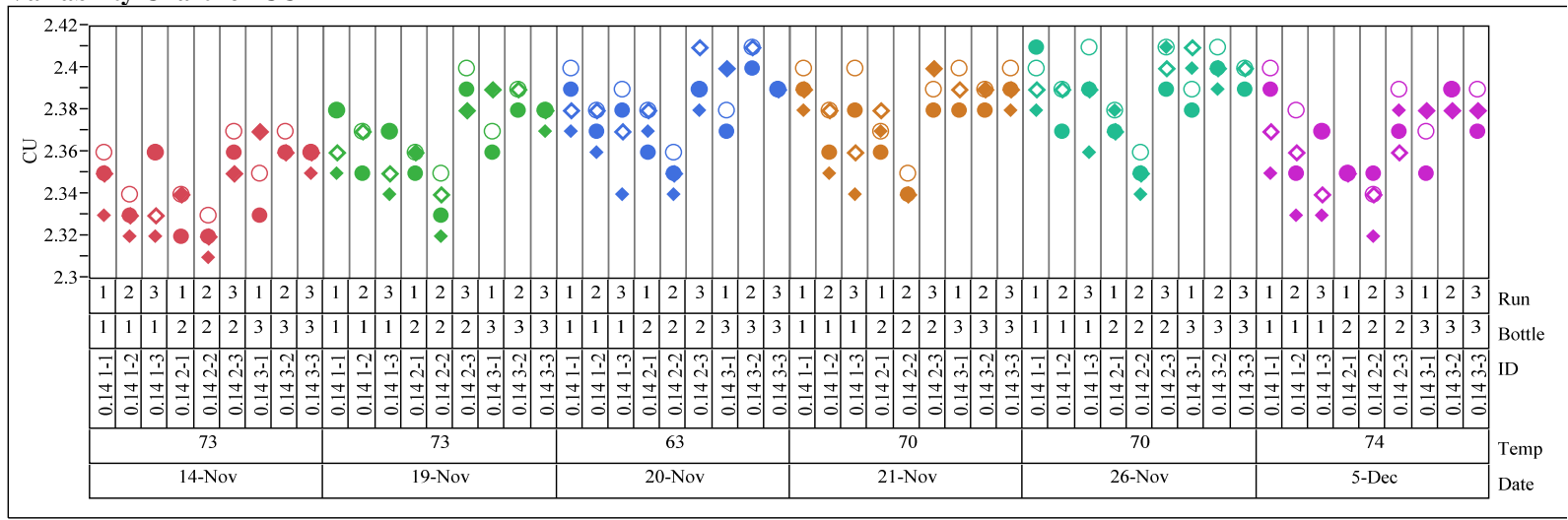

Meter=System 1, $\mathrm{Wt} \%=0.15$

Variability Chart for CU

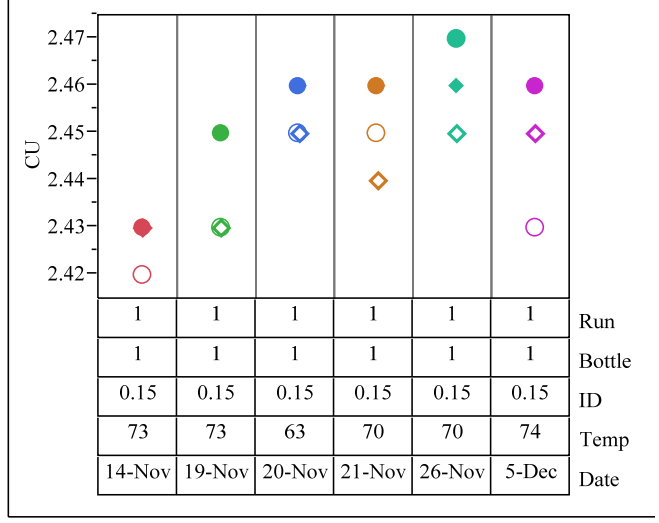


Exhibit A2. Concentration Unit (CU) Measurements by Meter for Deionized Water and Sludge Simulant Standards

Meter=System 1, Wt $\%=0.2$

$\circ$ - forward, $\bullet$ - backward, $\diamond-$ left, and $\bullet-$ right

Variability Chart for CU

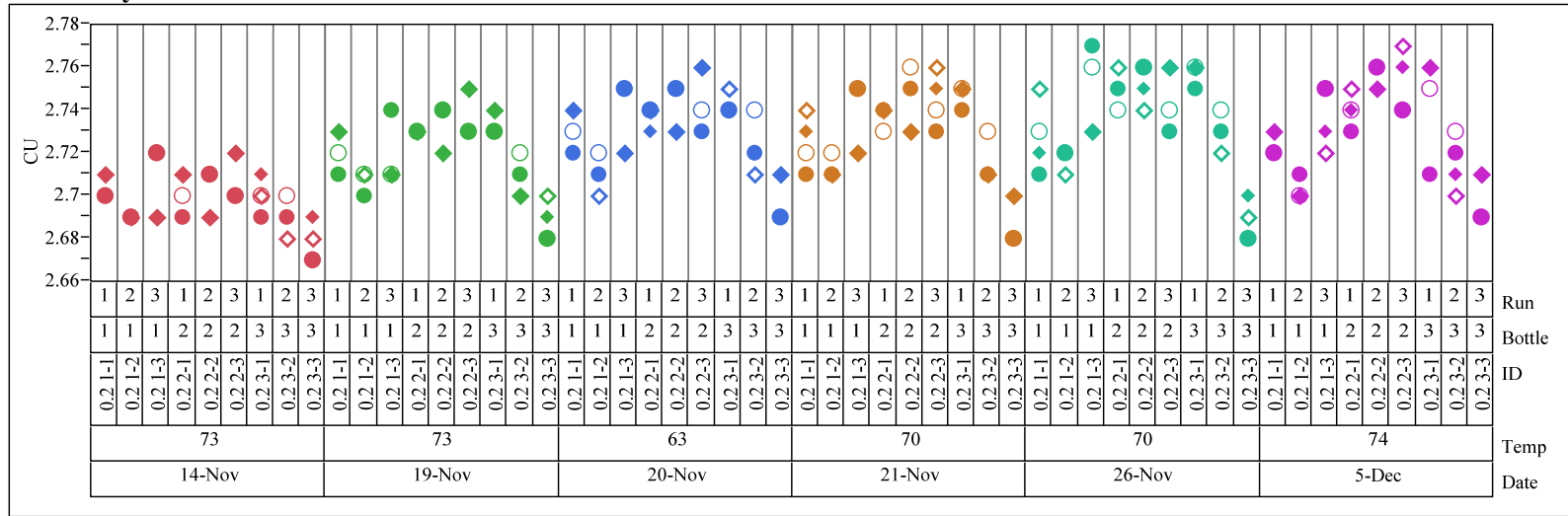

Meter=System 1, Wt $\%=0.3$

Variability Chart for CU

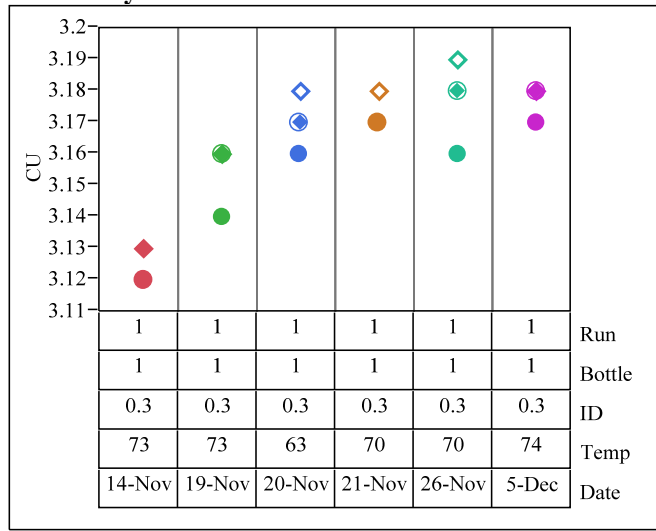

Meter=System 1, Wt $\%=0.4$

Variability Chart for CU

\begin{tabular}{|c|c|c|c|c|c|c|c|}
\hline $3.51-$ & & & & & & & \\
\hline $3.5-$ & & & $\diamond$ & & $\diamond$ & & \\
\hline $3.49-$ & & $\diamond$ & $\bullet$ & $\diamond$ & $\bullet$ & $\diamond$ & \\
\hline $3.48-$ & & $\bullet$ & & & & & \\
\hline $8^{3.47-}$ & & & 0 & & & - & \\
\hline$U_{3.46-}$ & $\diamond$ & O & - & - & - & 0 & \\
\hline $3.45-$ & & • & & & & & \\
\hline $3.44-$ & $\bullet$ & & & & & & \\
\hline $3.42-$ & & & & & & & \\
\hline & 1 & 1 & 1 & 1 & 1 & 1 & Run \\
\hline & 1 & 1 & 1 & 1 & 1 & 1 & Bottle \\
\hline & 0.4 & 0.4 & 0.4 & 0.4 & 0.4 & 0.4 & ID \\
\hline & 73 & 73 & 63 & 70 & 70 & 74 & Temp \\
\hline & 14-Nov & 19-Nov & $20-\mathrm{Nov}$ & $21-\mathrm{Nov}$ & $26-\mathrm{Nov}$ & 5-Dec & Date \\
\hline
\end{tabular}


Exhibit A2. Concentration Unit (CU) Measurements by Meter for Deionized Water and Sludge Simulant Standards

Meter=System 1, $\mathrm{Wt} \%=0.5$

$\circ$ - forward, $\bullet-$ backward, $\diamond-$ left, and $\bullet-$ right

Variability Chart for CU

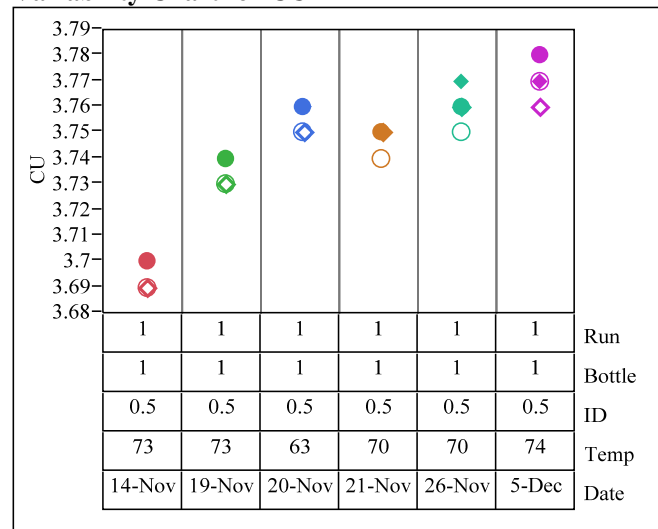

Meter=System 1, Wt $\%=0.6$

Variability Chart for CU

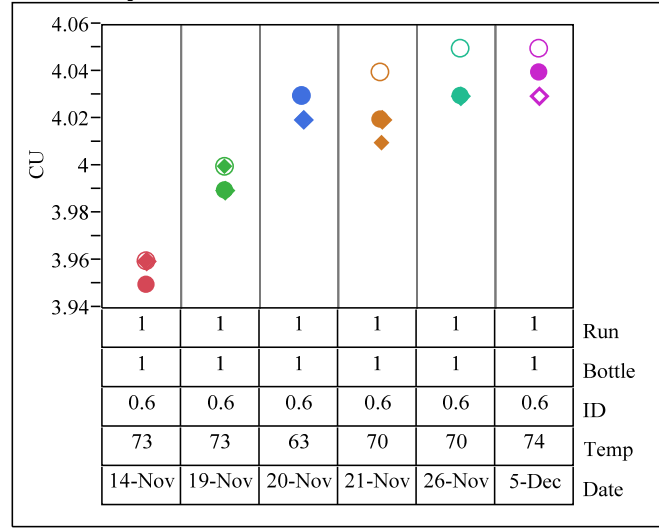

Meter=System 1, Wt $\%=0.7$

Variability Chart for CU

\begin{tabular}{|c|c|c|c|c|c|c|c|}
\hline $4.28-$ & & & & & & 9 & \\
\hline 4.24 & & & 8 & 8 & 8 & & \\
\hline $4.22-$ & & 8 & & & 0 & 0 & \\
\hline Z 4.2- & & & 0 & 0 & & & \\
\hline $\begin{array}{l}4.18- \\
4.16-\end{array}$ & 8 & 0 & & & & & \\
\hline $4.14-$ & 0 & & & & & & \\
\hline & 1 & 1 & 1 & 1 & 1 & 1 & Run \\
\hline & 1 & 1 & 1 & 1 & 1 & 1 & Bottle \\
\hline & 0.7 & 0.7 & 0.7 & 0.7 & 0.7 & 0.7 & ID \\
\hline & 73 & 73 & 63 & 70 & 70 & 74 & Temp \\
\hline & 14-Nov & 19-Nov & 20-Nov & 21-Nov & $26-\mathrm{Nov}$ & 5-Dec & Date \\
\hline
\end{tabular}


Exhibit A2. Concentration Unit (CU) Measurements by Meter for Deionized Water and Sludge Simulant Standards

Meter=System 1, Wt $\%=0.8$

$\circ$ - forward, $\bullet$ - backward, $\diamond-$ left, and $\bullet-$ right

Variability Chart for CU

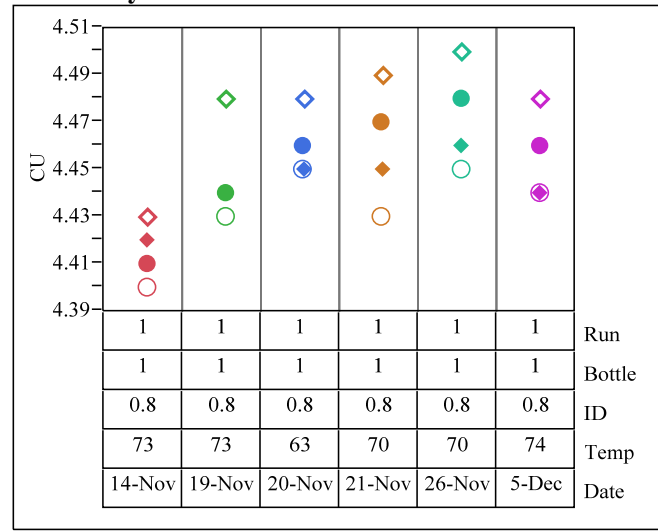

Meter=System 1, Wt\%=1.2

Variability Chart for CU

\begin{tabular}{|c|c|c|c|c|c|c|}
\hline & & 0 & 8 & 0 & $\diamond$ & \\
\hline \\
\hline
\end{tabular}

Meter=System 2, $\mathrm{Wt} \%=0$

Variability Chart for CU

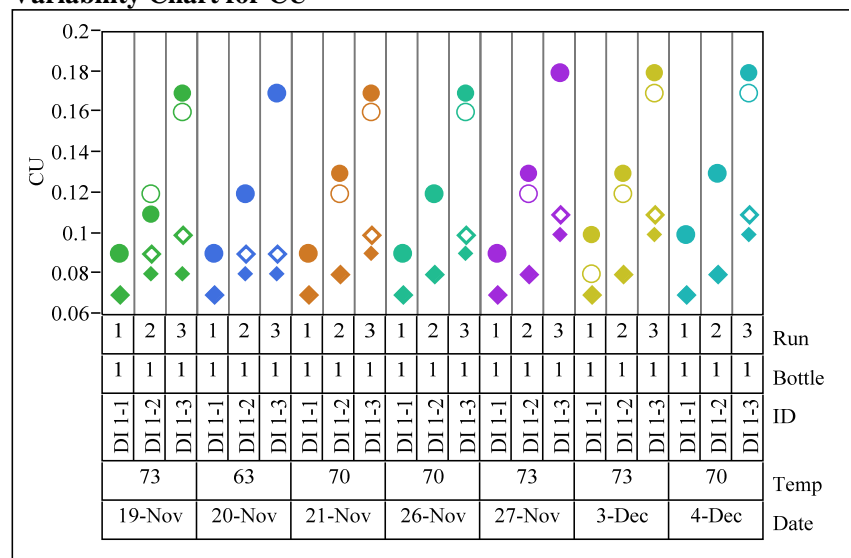


SRNL-STI-2013-00016

Revision 0

Exhibit A2. Concentration Unit (CU) Measurements by Meter for Deionized Water and Sludge Simulant Standards

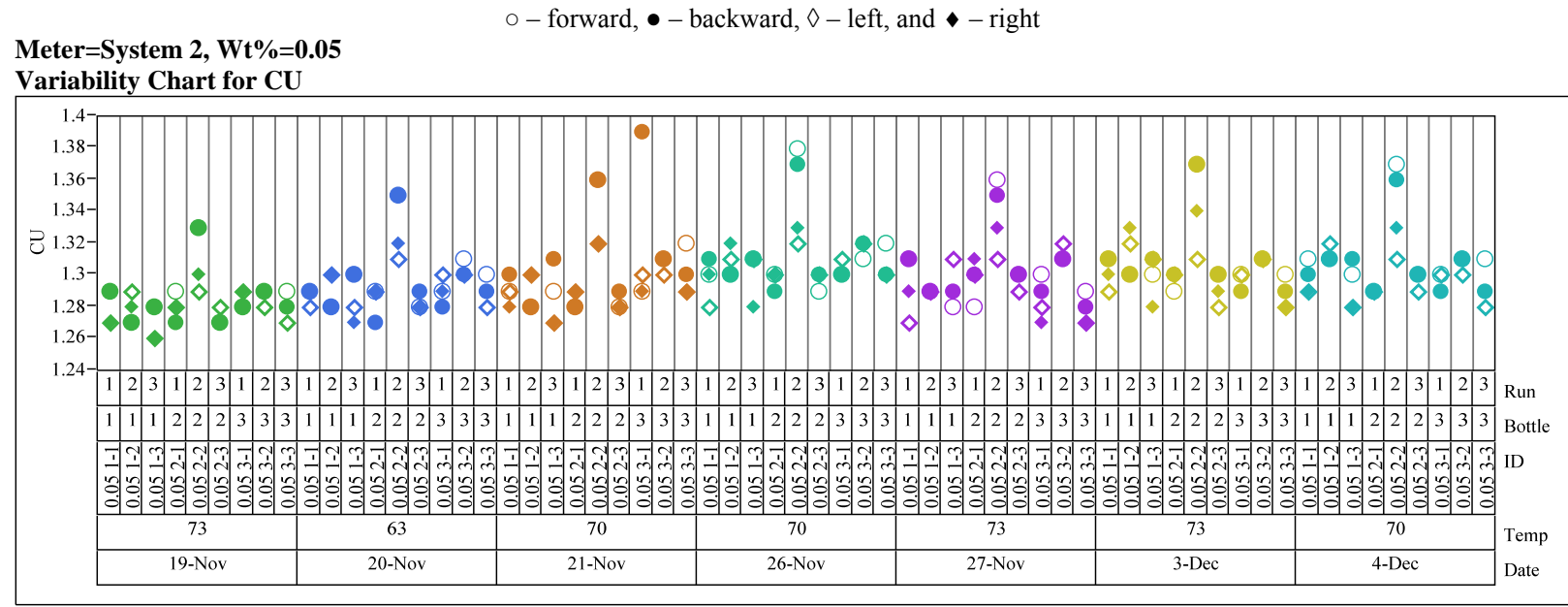

\section{Meter=System 2, Wt $\%=0.075$}

Variability Chart for $\mathrm{CU}$

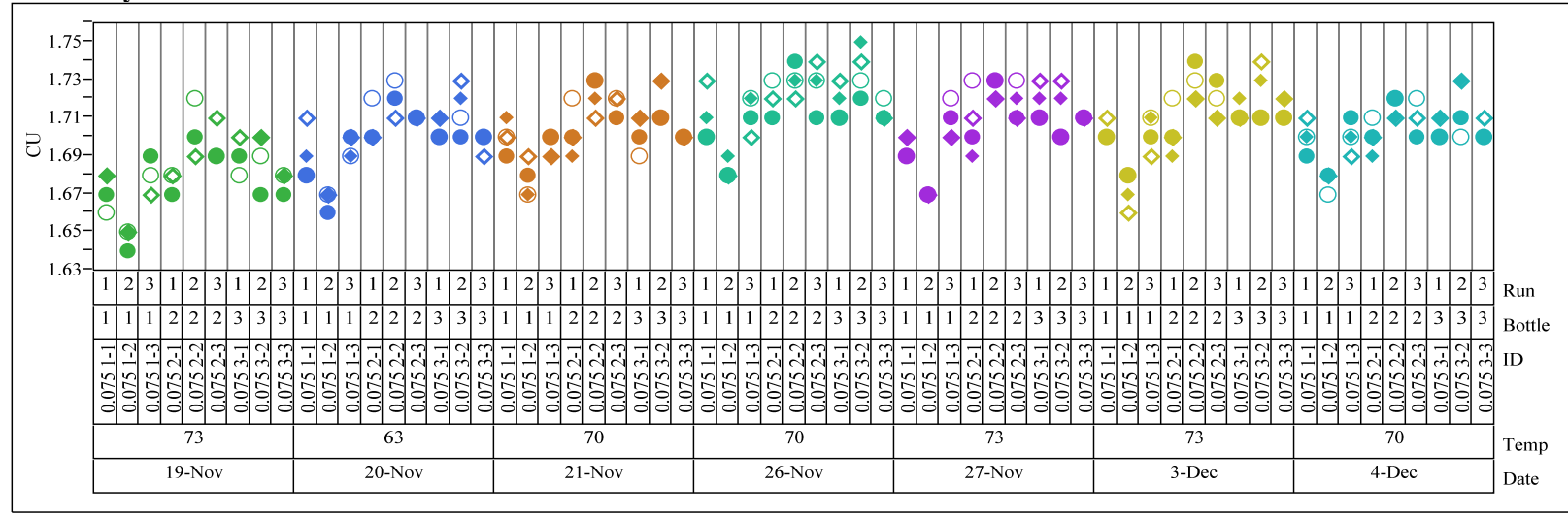

\section{Meter=System 2, $\mathbf{W t} \%=0.1$}

Variability Chart for CU

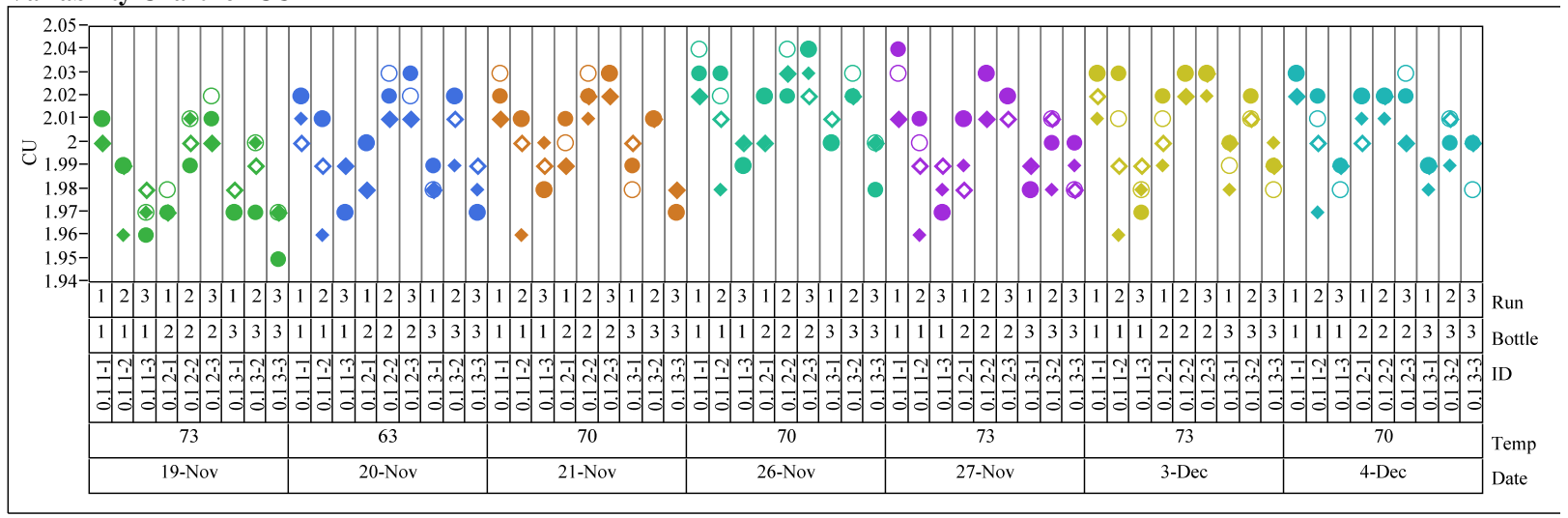


SRNL-STI-2013-00016

Revision 0

Exhibit A2. Concentration Unit (CU) Measurements by Meter for Deionized Water and Sludge Simulant Standards

Meter=System 2, $\mathrm{Wt} \%=0.14$

$\circ$ - forward, $\bullet$ - backward, $\diamond-$ left, and $\bullet-$ right

Variability Chart for CU

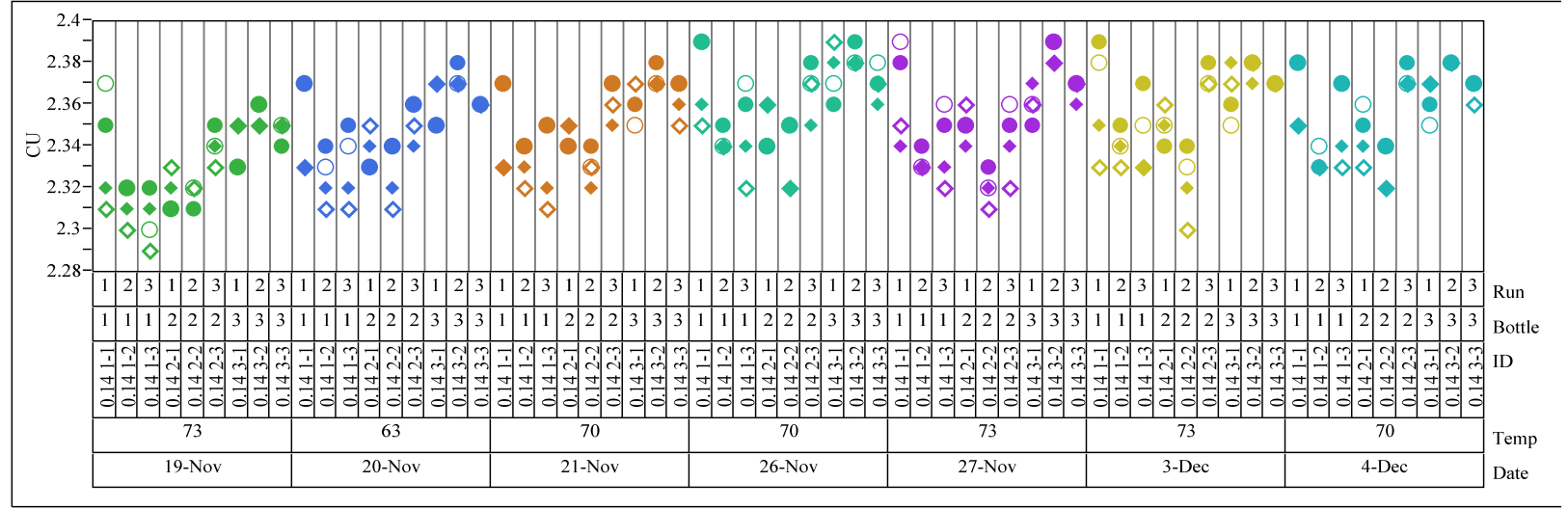

Meter=System 2, Wt\%=0.15

Variability Chart for CU

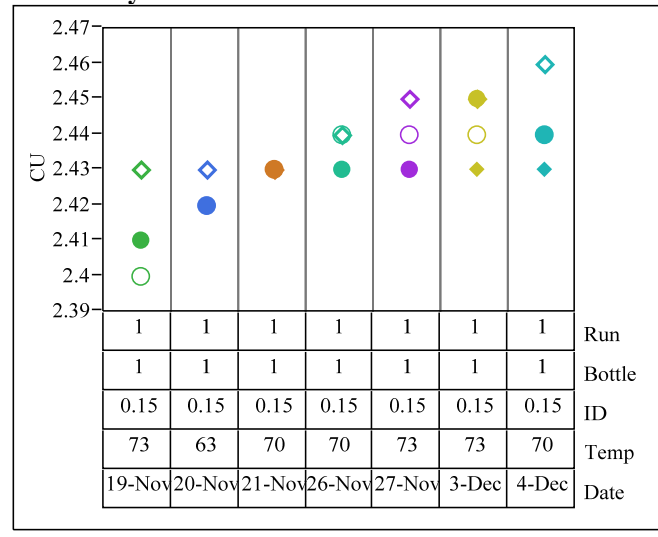

Meter=System $2, \mathrm{Wt} \%=0.2$

Variability Chart for CU

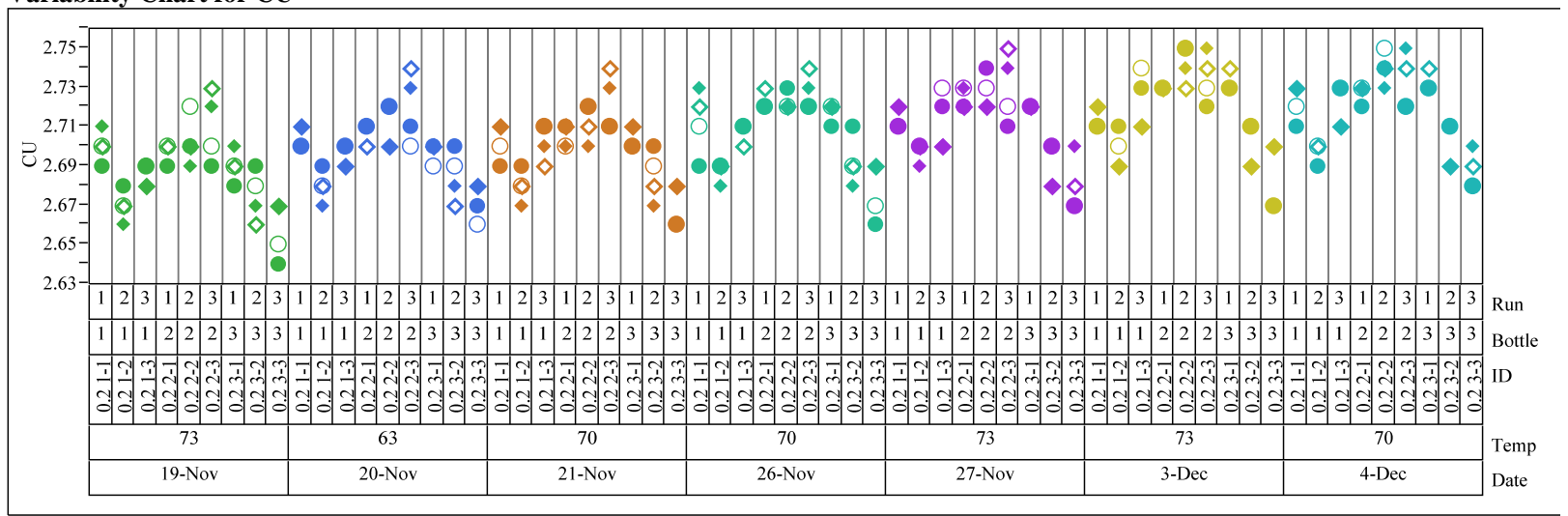


Exhibit A2. Concentration Unit (CU) Measurements by Meter for Deionized Water and Sludge Simulant Standards

Meter=System 2, Wt \% $=0.3$

$\circ$ - forward, $\bullet$ - backward, $\diamond-$ left, and $\bullet-$ right

Variability Chart for CU

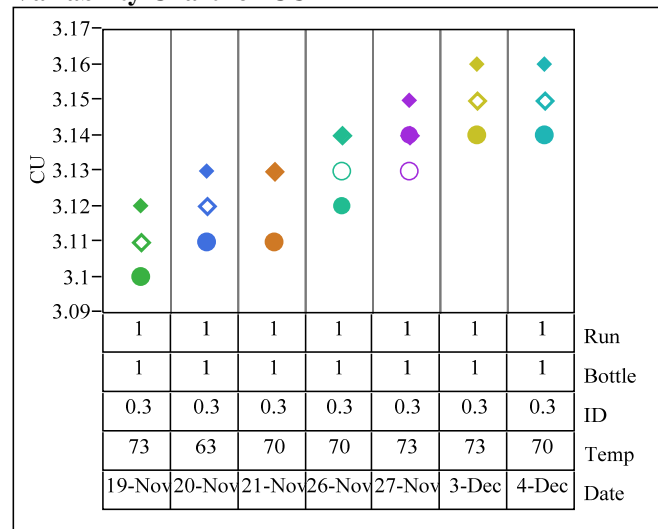

Meter=System 2, Wt $\%=0.4$

Variability Chart for CU

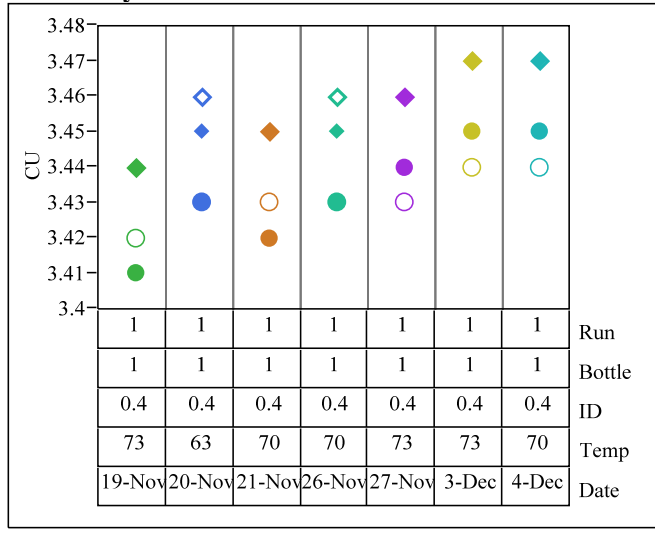

Meter=System 2, Wt $\%=0.5$

Variability Chart for CU

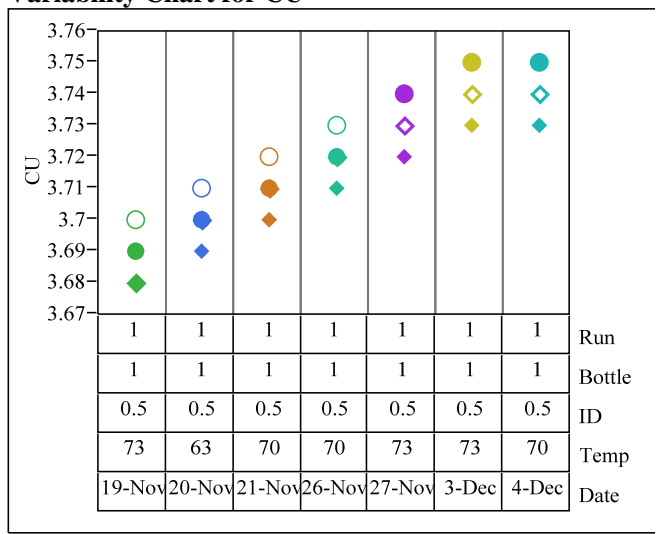


Exhibit A2. Concentration Unit (CU) Measurements by Meter for Deionized Water and Sludge Simulant Standards

Meter=System 2, Wt\%=0.6

$\circ$ - forward, $\bullet$ - backward, $\diamond-$ left, and $\bullet-$ right

Variability Chart for CU

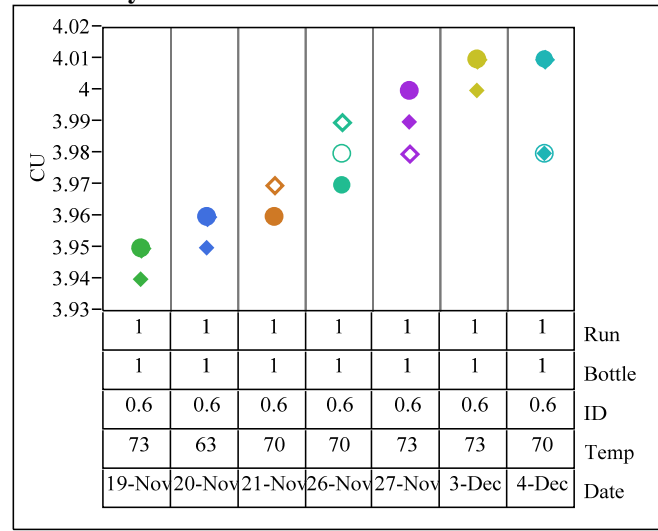

Meter=System 2, Wt $\%=0.7$

Variability Chart for CU

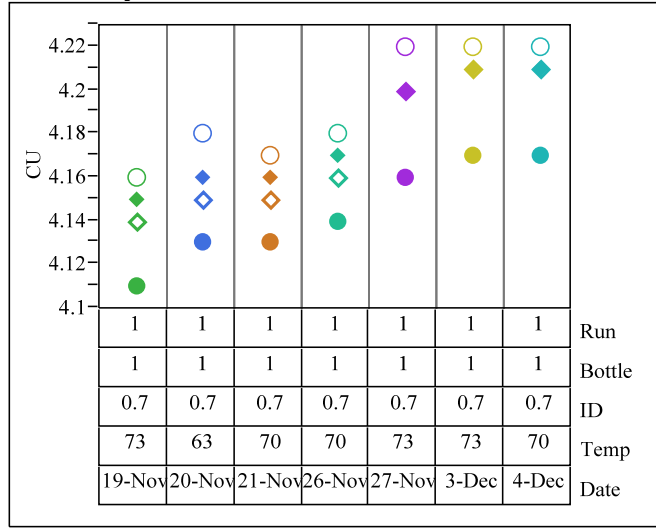

Meter=System 2, $\mathbf{W t} \%=0.8$

Variability Chart for CU

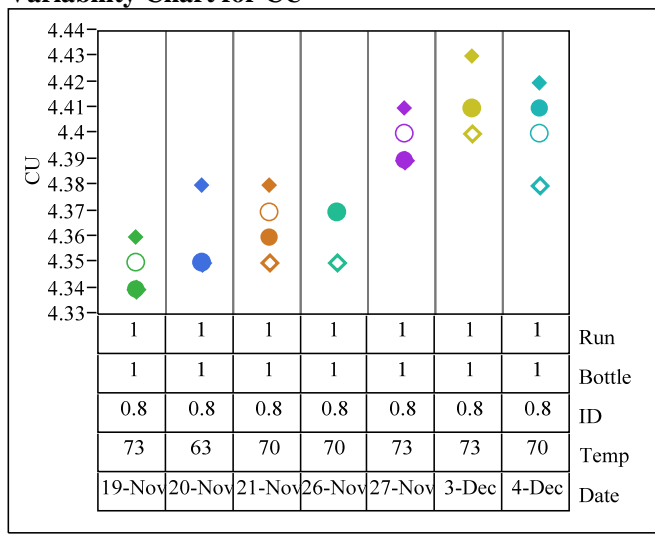


SRNL-STI-2013-00016

Revision 0

Exhibit A2. Concentration Unit (CU) Measurements by Meter for Deionized Water and Sludge Simulant Standards

Meter=System 2, Wt\%=1.2

$\circ$ - forward, $\bullet$ - backward, $\diamond-$ left, and $\bullet$ - right

Variability Chart for CU

\begin{tabular}{|c|c|c|c|c|c|c|c|c|}
\hline $4.84-$ & & & & & & & & \\
\hline $4.82-$ & & & & & $\bullet$ & & & \\
\hline $4.8-$ & & & $\bullet$ & & 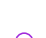 & , & $\bullet$ & \\
\hline $\begin{array}{r}4.78- \\
476-\end{array}$ & & $\bullet$ & & $\overrightarrow{0}$ & 0 & 8 & 0 & \\
\hline$\overbrace{4.74-}^{4.16^{-}}$ & $\bullet$ & 0 & 0 & 0 & & & 8 & \\
\hline 4.72 & 0 & 8 & - & 0 & & & & \\
\hline $4.7-$ & - & & $\diamond$ & & & & & \\
\hline $4.68-$ & $\diamond$ & & & & & & & \\
\hline 4.000 & 1 & 1 & 1 & 1 & 1 & 1 & 1 & Run \\
\hline & 1 & 1 & 1 & 1 & 1 & 1 & 1 & Bottle \\
\hline & 1.2 & 1.2 & 1.2 & 1.2 & 1.2 & 1.2 & 1.2 & ID \\
\hline & 73 & 63 & 70 & 70 & 73 & 73 & 70 & Temp \\
\hline & $19-\mathrm{Nc}$ & $20-\mathrm{No}$ & $1-\mathrm{No}$ & 6-No & 7-No & 3-Dec & 4-Dec & Date \\
\hline
\end{tabular}


Exhibit A3. Linear Model of CU Values versus Natural Logarithm of Wt\% Solids Values

Bivariate Fit of CU By ln(std wt\%) Meter=System 1, Date=14-Nov, Position=Back

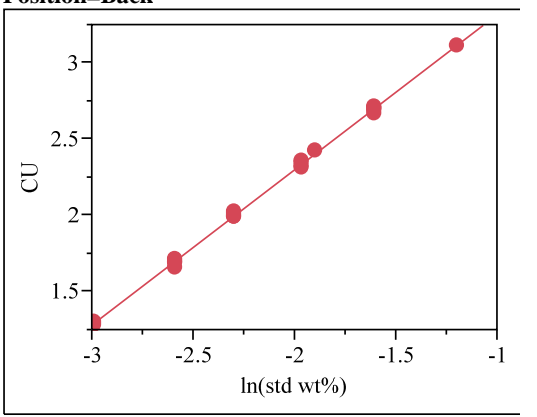

\section{Linear Fit}

Linear Fit

$\mathrm{CU}=4.3416961+1.0179849 * \ln (\mathrm{std} w \mathrm{w} \%)$

\section{Summary of Fit}

RSquare

Root Mean Square Error 0.999088

Mean of Response 2.039787

Observations (or Sum Wgts) $\quad 47$

$\begin{array}{lrrrr}\begin{array}{l}\text { Lack Of Fit } \\ \text { Source }\end{array} & \text { DF } & \text { Sum of Squares } & \text { Mean Square } & \text { F Ratio } \\ \text { Lack Of Fit } & 5 & 0.00173532 & 0.000347 & 1.5274 \\ \text { Pure Error } & 40 & 0.00908889 & 0.000227 & \begin{array}{r}\text { Prob }>\text { F } \\ \text { Total Error }\end{array} \\ & 45 & 0.01082421 & 0.2031 \\ & & & & \text { Max RSq } \\ & & 0.9993\end{array}$

Analysis of Variance

Analysis of Variance
Source DF Sum of Squares Mean Square F Ratio

$\begin{array}{crrrr}\text { Source } & \text { DF } & \text { Sum of Squares } & \text { Mean Square } & \text { F Ratio } \\ \text { Model } & 1 & 12.127674 & 12.1277 & 50418.96\end{array}$

$\begin{array}{lrrrrr}\text { Model } & 1 & 12.127674 & 12.1277 & 50418.96 \\ \text { Error } & 45 & 0.010824 & 0.000241 & \text { Prob > F } \\ \text { C. } & & & 12.13848 & & <.000\end{array}$

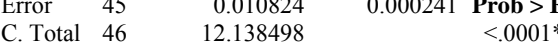

\section{Parameter Estimates}

Term Estimate Std Error t Ratio Prob $>\mid t$

$\begin{array}{llllll}\text { Intercept } & 4.3416961 & 0.010498 & 413.56 & <.0001\end{array}$

$\begin{array}{llllll}\ln \left(\text { std } w \omega^{\circ} \%\right) & 1.0179849 & 0.004534 & 224.54<.0001 *\end{array}$
Bivariate Fit of CU By $\ln ($ std wt \%) Meter=System 1, Date=14-Nov, Position=Frwrd

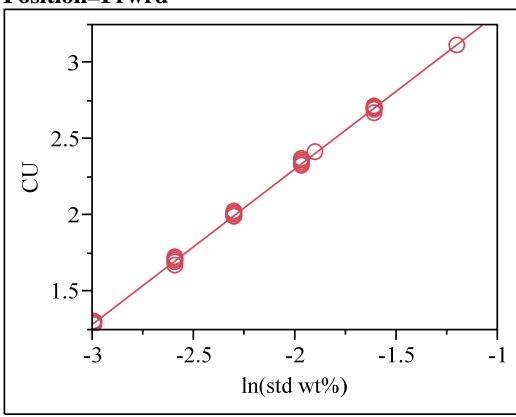

\section{Linear Fit}

Linear Fit

$\mathrm{CU}=4.3497194+1.019369 * \ln ($ std $\mathrm{wt} \%)$

\section{Summary of Fit}

RSquare

RSquare Adj

Root Mean Square

$\begin{array}{lr}\text { Mean of Response } & 2.044681 \\ \text { Observations (or Sum Wgts) } & 47\end{array}$

\subsection{8}

0.999118 0.01527

\begin{tabular}{lrrrr}
\multicolumn{2}{l}{ Lack Of Fit } & & & \\
Source & DF & Sum of Squares & Mean Square & F Ratio \\
Lack Of Fit & 5 & 0.00269657 & 0.000539 & 2.7657 \\
Pure Error & 40 & 0.00780000 & 0.000195 & $\begin{array}{r}\text { Prob }>\text { F } \\
\text { Total Error }\end{array}$ \\
& 45 & 0.01049657 & $0.0308^{*}$ \\
& & & & Max RSq \\
& & & 0.9994
\end{tabular}

Analysis of Variance
Source DF Sum of Squares Mean Square F Ratio $\begin{array}{lrrrr}\text { Source } & \text { DF } & \text { Sum of Squares } & \text { Mean Square } & \text { F Ratio } \\ \text { Model } & 1 & 12.160674 & 12.1607 & 52134.18\end{array}$ \begin{tabular}{lrrrr} 
Model & 1 & 12.160674 & 12.1607 & 52134.18 \\
Error & 45 & 0.010497 & 0.000233 & Prob $>$ F \\
\hline & & 12.17170 & & $<000$.
\end{tabular} $\begin{array}{lrrrr}\text { Error } & 45 & 0.010497 & 0.000233 & \begin{array}{l}\text { Prob }>\text { F } \\ \text { C. Total }\end{array} \\ & 46 & 12.171170 & <.0001^{*}\end{array}$

\section{Parameter Estimates}

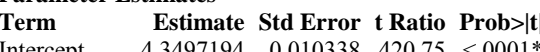

$\begin{array}{lllll} & \text { Entercept } & 4.3497194 & 0.010338 & 420.75<<.0001 *\end{array}$
Bivariate Fit of CU By $\ln ($ std wt\%) Meter=System 1, Date=14-Nov, Position=Left

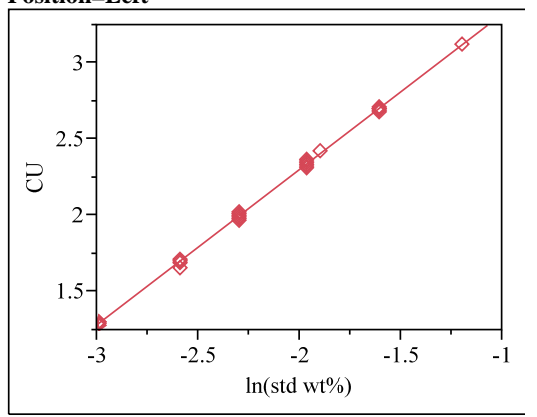

\section{- Linear Fit}

Linear Fit

$\mathrm{CU}=4.3433325+1.0182381 * \ln (\mathrm{std} w \mathrm{wt} \%)$

Summary of Fit

RSquare

$\begin{array}{ll}0.999024 \\ \text { RSquare Adj } & 0.999002\end{array}$

RSquare Adj $\quad 0.999002$

Meot Mean Square Eror $\quad 0.016233$

$\begin{array}{lr}\text { Mean of Response } & 2.040851 \\ \text { Observations (or Sum Wgts) } & 47\end{array}$

\section{Lack Of Fit}

Source DF Sum of Squares Mean Square F Ratio $\begin{array}{lllll}\text { Lack Of Fit } & 5 & 0.00136960 & 0.000274 & 1.0446\end{array}$

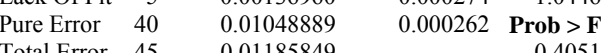
$\begin{array}{rrrr}\text { Total Error } 45 & 0.01185849 & 0.4051 \\ & & \text { Max RSq }\end{array}$ 0.9991

Analysis of Variance

Source DF Sum of Squares Mean Square F Ratio $\begin{array}{lllll}\text { Model } & 1 & 12.133707 & 12.1337 & 46044.38\end{array}$ $\begin{array}{lrrr}1 & 0.011858 & 0.000264 \text { Prob }>\text { F }\end{array}$

\section{Parameter Estimates}

$\begin{array}{lll}\text { Parameter Estimates } & \\ \text { Term Estimate Std Error t Ratio Prob }>|t|\end{array}$

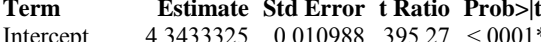

$\begin{array}{llllll}\ln (\mathrm{std} w \mathrm{w} \%) & 1.0182381 & 0.004745 & 214.58<.0001 & \end{array}$ 
Exhibit A3. Linear Model of CU Values versus Natural Logarithm of Wt\% Solids Values

Bivariate Fit of CU By ln(std wt\%) Meter=System 1, Date=14-Nov, Position=Right

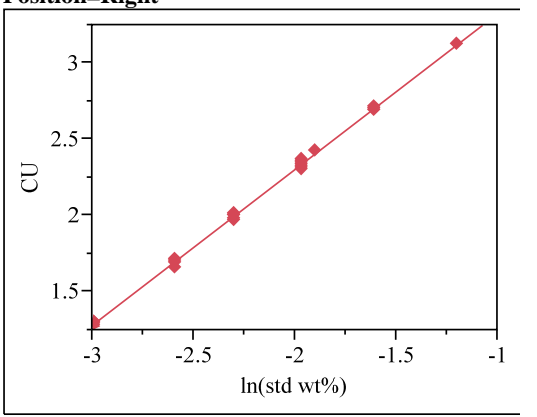

\section{Linear Fit}

Linear Fit

$\mathrm{CU}=4.3488173+1.0216046 * \ln (\mathrm{std} w \mathrm{w} \%)$

\section{Summary of Fit}

RSquare

0.99926

0.01609

$\begin{array}{lr}\text { Mean of Response } & 2.038723 \\ \text { Observations (or Sum Wgts) } & 47\end{array}$

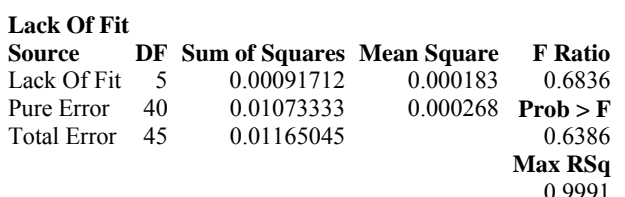

Analysis of Variance

Source DF Sum of Squares Mean Square F Ratio

$\begin{array}{lllll}\text { Model } & 1 & 12.214073 & 12.2141 & 47177.00\end{array}$

$\begin{array}{lll} & 0.011650 & 0.000259 \text { Prob }>\text { F }\end{array}$

C. Total $46 \quad 12.225723 \quad<.0001^{*}$

Parameter Estimates

Term Estimate Std Error t Ratio Prob $>\mid \mathrm{t}$

$\begin{array}{llrlll}\text { Intercept } & 4.3488173 & 0.010892 & 399.28 & <.0001 *\end{array}$

$\begin{array}{llllll}\ln \left(\text { std } w \omega^{\circ} \%\right) & 1.0216046 & 0.004703 & 217.20<.0001 *\end{array}$
Bivariate Fit of CU By $\ln ($ std wt \%) Meter=System 1, Date=19-Nov, Position=Back

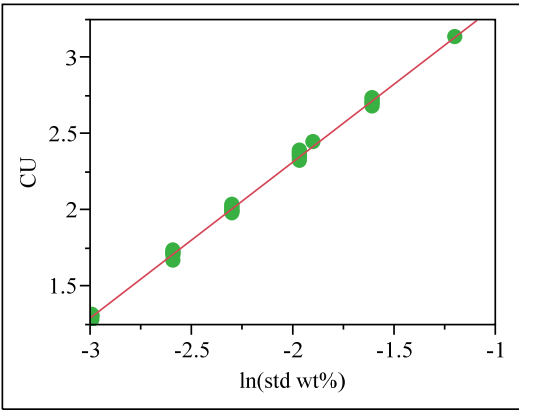

\section{Linear Fit}

Linear Fit

$\mathrm{CU}=4.3694757+1.0223663 * \ln (\mathrm{std} \mathrm{wt} \%)$

Summary of Fit

RSquare

RSquare Adj

Root Mean Squa

Mean of Response

0.998702

0.998702

2.05766
Lack Of Fit
Source DF Sum of Squares Mean Square F Ratio
$\begin{array}{lllll}\text { Lack Of Fit } & 5 & 0.00103900 & 0.000208 & 0.5728\end{array}$
$\begin{array}{lllll}\text { Pure Error } 40 & 0.01451111 & 0.000363 & \text { Prob }>\text { F }\end{array}$

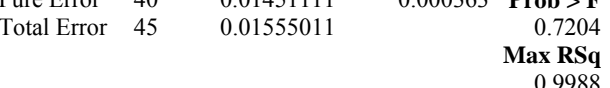

Analysis of Variance

Source DF Sum of Squares Mean Square F Ratio

$\begin{array}{lllll}\text { Model } & 1 & 12.232292 & 12.2323 & 35398.67\end{array}$

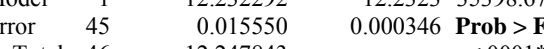

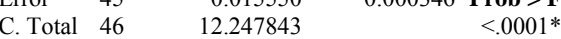

Parameter Estimates

Term Estimate Std Error t Ratio Prob $>|t|$

$\begin{array}{llrlll}\text { Intercept } & 4.3694757 & 0.012583 & 347.25 & <.0001 *\end{array}$

$\begin{array}{lllll}\ln (\mathrm{std} w \mathrm{w} \%) & 1.0223663 & 0.005434 & 188.15<0001^{*}\end{array}$
Bivariate Fit of CU By ln(std wt\%) Meter=System 1, Date=19-Nov, Position=Frwrd

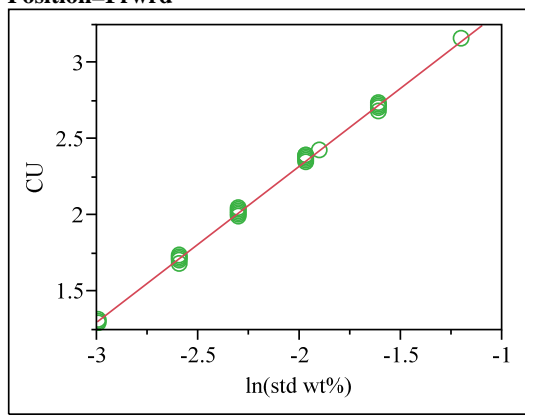

\section{- Linear Fit}

Linear Fit

$\mathrm{CU}=4.3761905+1.023548 * \ln (\mathrm{std} w \mathrm{wt} \%)$

Summary of Fit

RSquare

$\begin{array}{ll}0.998951 \\ \text { RSquare Adj } & 0.998928\end{array}$

RSquare Adj $\quad 0.998928$

Root Mean Square Eror -0.016916

$\begin{array}{lr}\text { Mean of Response } & 2.061702 \\ \text { Observations (or Sum Wgts) } & 47\end{array}$

Source DF Sum of Squares Mean Square F Ratio

$\begin{array}{lllll}\text { Lack Of Fit } & 5 & 0.00305447 & 0.000611 & 2.4878\end{array}$

$\begin{array}{llll}\text { Pure Error } & 40 & 0.00982222 & 0.000246 \text { Prob }>\text { F } \\ \text { To } & \end{array}$

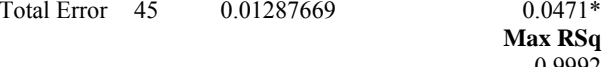

Analysis of Variance

Source DF Sum of Squares Mean Square F Ratio

$\begin{array}{llllll}\text { Model } & 1 & 12.260587 & 12.2606 & 42846.91\end{array}$

$\begin{array}{lrrr}\text { Error } 45 & 0.012877 & 0.000286 & \text { Prob }>\text { F } \\ \text { C. } & 46 & 12.27346 & \end{array}$

$\begin{array}{lrr}\text { C. Total } 46 & 12.273464 & 0.00286 \\ & <.0001^{*}\end{array}$

Parameter Estimates

Term Estimate Std Error t Ratio Prob $>\mid \mathbf{t}$

$\begin{array}{lrrrrr}\text { Intercept } & 4.3761905 & 0.01145 & 382.19<.0001^{*} \\ \text { In(std wt\%) } & 1.023548 & 0.004945 & 206.99<0001^{*}\end{array}$ 
Exhibit A3. Linear Model of CU Values versus Natural Logarithm of Wt\% Solids Values

Bivariate Fit of CU By ln(std wt\%) Meter=System 1, Date=19-Nov, Position=Left

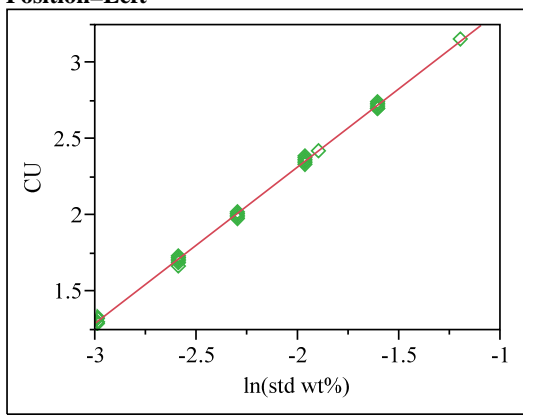

\section{Linear Fit}

Linear Fit

$\mathrm{CU}=4.3760624+1.0254673 * \ln (\mathrm{std} w \mathrm{w} \%)$

\section{Summary of Fit}

RSquare

RSquare Adj

0.998745

Tean 0.018336

Observations (or Sum Wgts) $\quad 47$

\begin{tabular}{lrrrr}
\multicolumn{2}{l}{ Lack Of Fit } & & & \\
Source & DF & Sum of Squares & Mean Square & F Ratio \\
Lack Of Fit & 5 & 0.00208503 & 0.000417 & 1.2787 \\
Pure Error & 40 & 0.01304444 & 0.000326 & Prob > F \\
Total Error & 45 & 0.01512947 & 0.2921 \\
& & & & Max RSq \\
& & & 0.9989
\end{tabular}

Analysis of Variance

Source DF Sum of Squares Mean Square F Ratio

$\begin{array}{lrrrr}\text { Model } & 1 & 12.306611 & 12.3066 & 36603.89 \\ \text { Error } & 45 & 0.015129 & 0.000336 & \text { Prob }>\text { F }\end{array}$

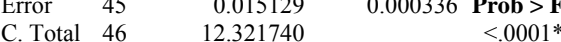

Parameter Estimates

$\begin{array}{lrrrrr}\text { Term } & \text { Estimate } & \text { Std Error } & \text { t Ratio } & \text { Prob }>|\mathbf{t}| \\ \text { Intercept } & 4.3760624 & 0.012412 & 352.58 & <.0001^{*}\end{array}$

$\begin{array}{lrrrrr}\ln \left(\text { std } w t^{\circ} \%\right) & 1.0254673 & 0.00536 & 191.32<.0001 *\end{array}$
Bivariate Fit of CU By $\ln ($ std wt \%) Meter=System 1, Date=19-Nov, Position=Right

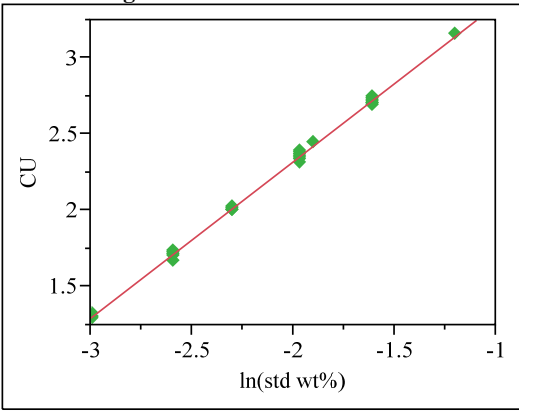

\section{Linear Fit}

Linear Fit

$\mathrm{CU}=4.375093+1.0256032 * \ln (\mathrm{std} \mathrm{wt} \%)$

\section{Summary of Fit}

RSquare

$\begin{array}{ll}\text { RSquare Adj } & 0.998834 \\ \text { Root Mean Square Error } & 0.017676\end{array}$

Mean of Response -0.017676

Observations (or Sum Wgts) $\quad 47$

$\begin{array}{lrrrr}\begin{array}{l}\text { Lack Of Fit } \\ \text { Source }\end{array} & \text { DF } & \text { Sum of Squares } & \text { Mean Square } & \text { F Ratio } \\ \text { Lack Of Fit } & 5 & 0.00114909 & 0.000230 & 0.7120 \\ \text { Pure Error } & 40 & 0.01291111 & 0.000323 & \begin{array}{l}\text { Prob }>\text { F } \\ \text { Total Error }\end{array} \\ & 45 & 0.01406020 & 0.6180 \\ & & & & \text { Max RSq } \\ & & 0.9990\end{array}$

Analysis of Variance
Source DF Sum of Squares Mean Square F Ratio

$\begin{array}{lrrrr}\text { Source } & \text { DF } & \text { Sum of Squares Mean Square } & \text { F Ratio } \\ \text { Model } & 1 & 12.309872 & 12.3099 & 39398.03\end{array}$

$\begin{array}{lrrrrr}\text { Model } & 1 & 12.309872 & 12.3099 & 39398.03 \\ \text { Error } & 45 & 0.014060 & 0.000312 & \text { Prob }>\text { F } \\ \text { C. } & & & \end{array}$

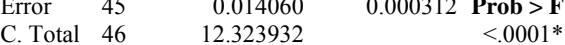

\section{Parameter Estimates}

Term Estimate Std Error t Ratio Prob $>|t|$

$\begin{array}{lllllll}\text { Intercept } & 4.375093 & 0.011965 & 365.66<.0001^{*}\end{array}$

$\begin{array}{lrrrrr}\ln \left(\mathrm{std} w \mathrm{w}^{\circ} \%\right) & 1.0256032 & 0.005167 & 198.49<.0001^{*}\end{array}$
Bivariate Fit of CU By $\ln ($ std wt \%) Meter=System 1, Date=20-Nov, Position=Back

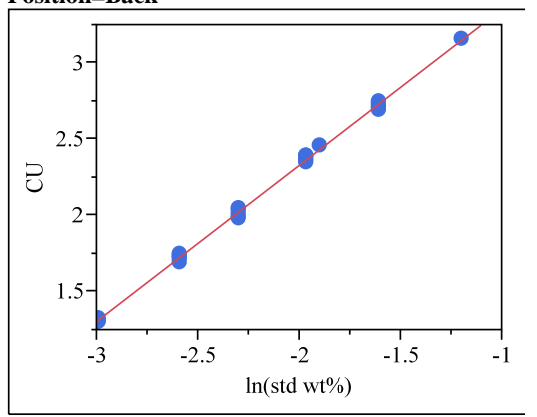

\section{- Linear Fit}

Linear Fit

$\mathrm{CU}=4.381622+1.0235036 * \ln (\mathrm{std} w \mathrm{wt} \%)$

Summary of Fit

RSquare

$\begin{array}{ll}0.998809 \\ \text { RSquare Adj } & 0.998783\end{array}$

Root Mean Square Error $\quad 0.998783$

Root Mean Square Error -0.018023

$\begin{array}{lr}\text { Mean of Response } & 2.067234 \\ \text { Observations (or Sum Wgts) } & 47\end{array}$

\section{Lack Of Fit}

Source DF Sum of Squares Mean Square F Ratio

$\begin{array}{lllll}\text { Lack Of Fit } & 5 & 0.00186195 & 0.000372 & 1.1678\end{array}$

$\begin{array}{lllll}\text { Pure Error } & 40 & 0.01275556 & 0.000319 & \text { Prob }>\text { F } \\ & & & 0.3419\end{array}$

$\begin{array}{llrr}\text { Total Error } & 45 & 0.01461750 & 0.3419 \\ & & & \text { Max RSq } \\ & & \end{array}$

Analysis of Variance

Source DF Sum of Squares Mean Square F Ratio

Model $\begin{array}{llll}1 & 12.259523 & 12.2595 & 37740.96\end{array}$

$\begin{array}{lrrr}\text { Error } 45 & 0.014618 & 0.000325 \text { Prob }>\text { F }\end{array}$

$\begin{array}{lrr}\text { C. Total } 46 & 12.274140 & 0.00325 \\ & <.0001^{*}\end{array}$

Term Estimate Std Error t Ratio Prob $>|\mathbf{t}|$

$\begin{array}{lrrrr}\text { Intercept } & 4.381622 & 0.0122 & 359.15 & <.0001^{*} \\ \ln (\mathrm{std} w \mathrm{wt} \%) & 1.0235036 & 0.005268 & 194.27 & <.0001^{*}\end{array}$ 
Exhibit A3. Linear Model of CU Values versus Natural Logarithm of Wt\% Solids Values

Bivariate Fit of CU By ln(std wt \%) Meter=System 1, Date=20-Nov,

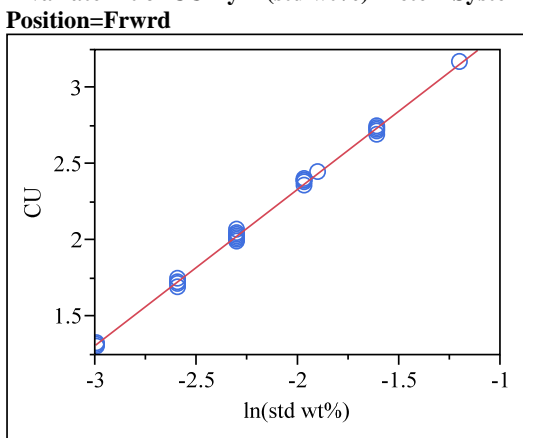

\section{Linear Fit}

Linear Fit

$\mathrm{CU}=4.3921487+1.0253361 * \ln (\mathrm{std} w \mathrm{w} \%)$

\section{Summary of Fit}

RSquare

RSquare Adj

\begin{tabular}{ll}
0.017399 \\
\hline
\end{tabular}

2.073617

\section{Lack Of Fit}

Source DF Sum of Squares Mean Square F Ratio

$\begin{array}{lllll}\text { Lack Of Fit } & 5 & 0.00280071 & 0.000560 & 2.0703\end{array}$

$\begin{array}{lllll}\text { Pure Error } 40 & 0.01082222 & 0.000271 \quad \text { Prob }>\text { F }\end{array}$

$\begin{array}{llrr}\text { Total Error } \quad 45 & 0.01362294 & 0.000271 & \text { Prob } \\ & & & 0.0894\end{array}$

Max RSq
0.9991

Analysis of Variance
Source DF Sum of Squares Mean Square F Ratio

$\begin{array}{crrrr}\text { Source } & \text { DF } & \text { Sum of Squares } & \text { Mean Square } & \text { F Ratio } \\ \text { Model } & 1 & 12.303462 & 12.3035 & 40641.44\end{array}$

$\begin{array}{lrrrrr}\text { Model } & 1 & 12.303462 & 12.3035 & 40641.44 \\ \text { Error } & 45 & 0.013623 & 0.000303 & \text { Prob }>\text { F }\end{array}$

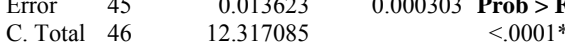

Parameter Estimates

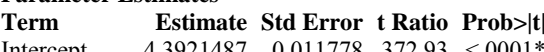

$\begin{array}{llllll} & \text { Estercept } & 4.3921487 & 0.011778 & 372.93<.0001^{*}\end{array}$
Bivariate Fit of CU By $\ln ($ std wt \%) Meter=System 1, Date=20-Nov, Position=Left

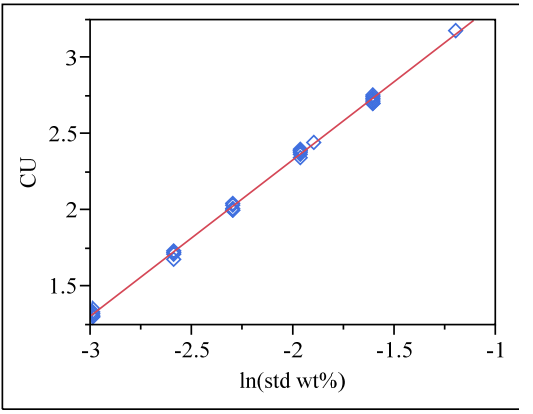

\section{Linear Fit}

Linear Fit

$\mathrm{CU}=4.388299+1.0249509 * \ln (\mathrm{std} \mathrm{wt} \%)$

\section{Summary of Fit}

RSquare

RSquare Adj

Root Mean Square Error

0.998533
0.9985
0.020034
2.070638

\section{Lack Of Fit}

Source DF Sum of Squares Mean Square F Ratio

$\begin{array}{lrlll}\text { Lack Of Fit } & 5 & 0.00375021 & 0.000750 & 2.0964\end{array}$

$\begin{array}{lllll}\text { Pure Error } & 40 & 0.01431111 & 0.000358 & \text { Prob }>\text { F } \\ \text { Tota } & \end{array}$

$\begin{array}{rrrr}\text { Total Error } 45 \quad 0.01806132 & 0.0859 \\ & & & 0.9988\end{array}$

Analysis of Variance

Source DF Sum of Squares Mean Square F Ratio

$\begin{array}{cccrr}\text { Source } & \text { DF } & \text { Sum of Squares } & \text { Mean Square } & \text { F Ratio } \\ \text { Model } & 1 & 12.294220 & 12.2942 & 30631.20\end{array}$

$\begin{array}{lrrrrr}\text { Model } & 1 & 12.294220 & 12.2942 & 30631.20 \\ \text { Error } & 45 & 0.018061 & 0.000401 & \text { Prob }>\text { F }\end{array}$

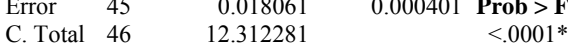

\section{Parameter Estimates}

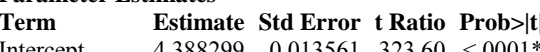

$\begin{array}{lllllll} & \text { Estercept } & 4.388299 & 0.013561 & 323.60< & <.0001 *\end{array}$
Bivariate Fit of CU By $\ln ($ std wt \%) Meter=System 1, Date=20-Nov, Position=Right

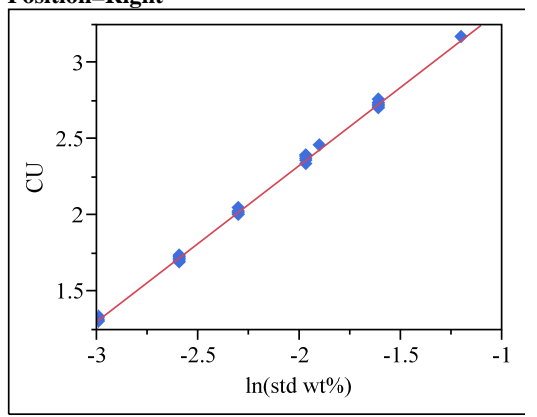

\section{- Linear Fit}

Linear Fit

$\mathrm{CU}=4.3829694+1.0244758^{*} \ln (\mathrm{std} w \mathrm{w} \%)$

Summary of Fit

RSquare $\quad 0.998987$

0.998964

Root Mean Square Error $\quad 0.01664$

$\begin{array}{lr}\text { Mean of Response } & 2.066383 \\ \text { Observations (or Sum Wgts) } & 47\end{array}$

\section{Lack Of Fit}

Source DF Sum of Squares Mean Square F Ratio $\begin{array}{lrlll}\text { Lack Of Fit } \quad 5 & 0.00165982 & 0.000332 & 1.2295\end{array}$ $\begin{array}{lllll}\text { Pure Error } & 40 & 0.01080000 & 0.000270 \quad \text { Prob }>\text { F } \\ \text { Total Error } & 45 & 0.01245982 & 0.3133\end{array}$

$\begin{array}{rrrr}\text { Total Error } & 45 & 0.01245982 & 0.3133 \\ & & & 0.9991\end{array}$

Analysis of Variance

Source DF Sum of Squares Mean Square F Ratio

$\begin{array}{lllll}\text { Model } & 1 & 12.282825 & 12.2828 & 44360.77\end{array}$

$\begin{array}{lrrr} & 1 & 0.012460 & 0.000277 \text { Prob }>\text { F }\end{array}$

Parameter Estimates

$\begin{array}{llllll}\text { Term } & \text { Estimate } & \text { Std Error } & \text { t Ratio } & \text { Prob }>|\mathbf{t}| \\ \text { Intercept } & 4.3829694 & 0.011264 & 389.13 & <.0001^{*}\end{array}$

$\begin{array}{llllll}\text { Intercept } & 4.3829694 & 0.011264 & 389.13 & <.0001 \\ \ln (\mathrm{std} w \mathrm{wt} \%) & 1.0244758 & 0.004864 & 210.62 & <.0001^{*}\end{array}$ 
Exhibit A3. Linear Model of CU Values versus Natural Logarithm of Wt\% Solids Values

Bivariate Fit of CU By ln(std wt\%) Meter=System 1, Date=21-Nov, Position=Back

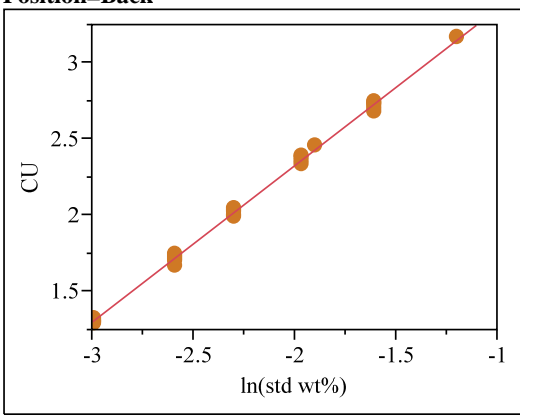

\section{Linear Fit}

Linear Fit

$\mathrm{CU}=4.3848746+1.0261652 * \ln (\mathrm{std} w \mathrm{w} \%)$

\section{Summary of Fit}

RSquare

Root Mean Square Error $\quad 0.998526$

Mean of Response 2.064468

Observations (or Sum Wgts) $\quad 47$

\begin{tabular}{lrrrr}
\multicolumn{2}{l}{ Lack Of Fit } & & & \\
Source & DF & Sum of Squares & Mean Square & F Ratio \\
Lack Of Fit & 5 & 0.00230464 & 0.000461 & 1.1903 \\
Pure Error & 40 & 0.01548889 & 0.000387 & Prob $>$ F \\
Total Error & 45 & 0.01779353 & 0.3312 \\
& & & & Max RSq \\
& & & 0.9987
\end{tabular}

Analysis of Variance

Source DF Sum of Squares Mean Square F Ratio

Model $\quad 1 \quad 12.323368 \quad 12.3234 \quad 31165.92$

Eror $45-0.017794-0.000395$ Prob $>$ F

\begin{tabular}{lrr} 
C. Total 46 & 12.341162 & 0.00395 \\
\hline & $<.0001^{*}$
\end{tabular}

Parameter Estimates

Term Estimate Std Error t Ratio Prob $>\mid t$

$\begin{array}{lrrrr}\text { Intercept } & 4.3848746 & 0.01346 & 325.77<.0001\end{array}$

$\begin{array}{llllll}\ln \left(\text { std } w \omega^{\circ} \%\right) & 1.0261652 & 0.005813 & 176.54<.0001 *\end{array}$
Bivariate Fit of CU By $\ln ($ std wt \%) Meter=System 1, Date=21-Nov, Position=Frwrd

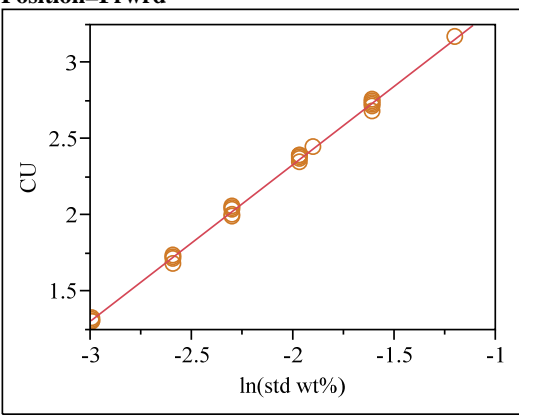

\section{Linear Fit}

Linear Fit

$\mathrm{CU}=4.394876+1.027295 * \ln (\mathrm{std} w \mathrm{w} \%)$

Summary of Fit

RSquare

RSquare Adj

Root Mean Square

0.019895 0.019895

Observations (or Sum Wgts) $\quad 47$

\section{Lack Of Fit \\ Source DF Sum of Squares Mean Square F Ratio \\ $\begin{array}{lllll}\text { Lack Of Fit } & 5 & 0.00338846 & 0.000678 & 1.8796\end{array}$ \\ $\begin{array}{lllll}\text { Pure Error } 40 & 0.01442222 & 0.000361 & \text { Prob }>\text { F }\end{array}$

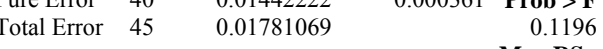 \\ Max RSq
0.9988}

Analysis of Variance

Source DF Sum of Squares Mean Square F Ratio

$\begin{array}{lllll}\text { Model } & 1 & 12.350517 & 12.3505 & 31204.48\end{array}$

$\begin{array}{llll} & & & \\ \text { Error } & 45 & 0.017811 & 0.000396 \text { Prob }>\text { F }\end{array}$

C. Total $46 \quad 12.368328$

$<.0001^{*}$

Parameter Estimates

Term Estimate Std Error t Ratio Prob $>\mid \mathbf{t}$

$\begin{array}{llllll}\text { Intercept } & 4.394876 & 0.013467 & 326.35 & <.0001 *\end{array}$

$\ln ($ std wt\%) $\quad 1.027295 \quad 0.005815 \quad 176.65<0001 *$
Bivariate Fit of CU By ln(std wt\%) Meter=System 1, Date=21-Nov, Position=Left

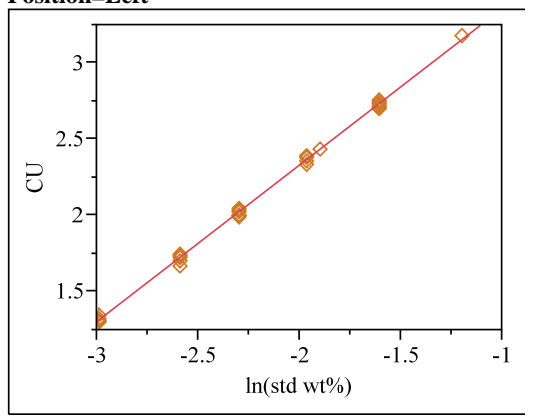

\section{- Linear Fit}

Linear Fit

$\mathrm{CU}=4.3872441+1.0256135 * \ln (\mathrm{std} w \mathrm{w} \%)$

Summary of Fit

RSquare $\quad 0.998507$

RSquare Adj $\quad 0.998474$

Root Mean Square Error $\quad 0.020225$

$\begin{array}{lr}\text { Mean of Response } & 2.068085 \\ \text { Observations (or Sum Wgts) } & 47\end{array}$

\section{Lack Of Fit}

Source DF Sum of Squares Mean Square F Ratio $\begin{array}{lrrrr}\text { Lack Of Fit } & 5 & 0.00260742 & 0.000521 & 1.3202\end{array}$ $\begin{array}{lllrr}\text { Pure Error } & 40 & 0.01580000 & 0.000395 & \text { Prob }>\text { F } \\ \text { Total Error } & 45 & 0.01840742 & 0.2752\end{array}$

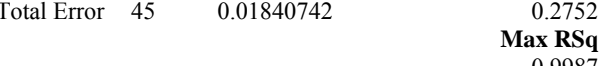

Analysis of Variance

Source DF Sum of Squares Mean Square F Ratio

$\begin{array}{lllll}\text { Model } & 1 & 12.310120 & 12.3101 & 30094.14\end{array}$

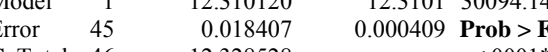

$\begin{array}{lll}\text { C. Total } 46<0001 * & 12.328528 & 0.00409\end{array}$

Term Estimate Std Error t Ratio Prob $>\mid \mathbf{t}$

$\begin{array}{lrrrr}\text { Intercept } & 4.3872441 & 0.01369 & 320.46 & <.0001^{*} \\ \text { In(std wt\%) } & 1.0256135 & 0.005912 & 173.48 & <.0001^{*}\end{array}$ 
Exhibit A3. Linear Model of CU Values versus Natural Logarithm of Wt\% Solids Values

Bivariate Fit of CU By ln(std wt\%) Meter=System 1, Date=21-Nov, Position=Right

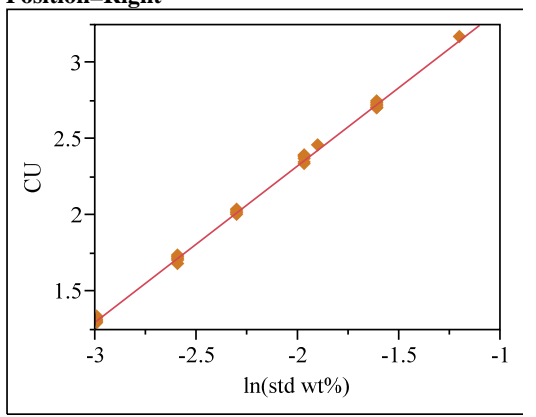

\section{Linear Fit}

Linear Fit

$\mathrm{CU}=4.3825256+1.0254087 * \ln (\operatorname{std} \mathrm{wt} \%)$

\section{Summary of Fit}

RSquare

RSquare Adj

Root Men Square Error $\quad 0.998763$

Tean of Respone Enor 0.018201

Observations (or Sum Wgts) $\quad 47$

\begin{tabular}{lrrrr}
$\begin{array}{l}\text { Lack Of Fit } \\
\text { Source }\end{array}$ & DF & Sum of Squares & Mean Square & F Ratio \\
Lack Of Fit & 5 & 0.00179564 & 0.000359 & 1.0956 \\
Pure Error & 40 & 0.01311111 & 0.000328 & $\begin{array}{r}\text { Prob }>\text { F } \\
\text { Total Error }\end{array}$ \\
\hline & 0.01450676 & & 0.3779 \\
& & & $\begin{array}{r}\text { Max RSq } \\
0.9989\end{array}$
\end{tabular}

Analysis of Variance

Source DF Sum of Squares Mean Square F Ratio

$\begin{array}{lllll}\text { Model } & 1 & 12.305204 & 12.3052 & 37146.52\end{array}$

$\begin{array}{lllll}1 & 12.305204 & 12.3052 & 37146.52 \\ \text { Error } & 45 & 0.014907 & 0.000331 \quad \text { Prob }>\text { F }\end{array}$

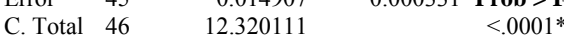

Parameter Estimates

Term Estimate Std Error t Ratio Prob $>|t|$

$\begin{array}{llllll}\text { Intercept } & 4.3825256 & 0.01232 & 355.73<.0001^{*}\end{array}$

$\begin{array}{lllll}\ln \left(\text { std } w \omega^{\circ} \%\right) & 1.0254087 & 0.00532 & 192.73<.0001^{*}\end{array}$
Bivariate Fit of CU By $\ln ($ std wt \%) Meter=System 1, Date=26-Nov, Position=Back

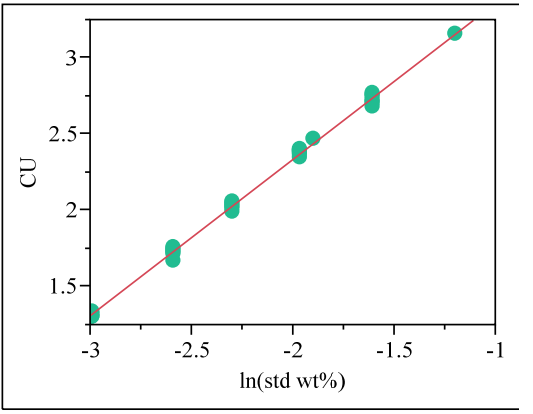

\section{Linear Fit}

Linear Fit

$\mathrm{CU}=4.3895418+1.0241832 * \ln (\mathrm{std} \mathrm{wt} \%)$

\section{Summary of Fit}

RSquare

RSquare Adj

Root Mean Square

$\begin{array}{lr}\text { Mean of Response } & 2.073617 \\ \text { Observations (or Sum Wgts) } & 47\end{array}$

0.998367

0.998331 0.021122

\begin{tabular}{lrrrr}
\multicolumn{2}{l}{ Lack Of Fit } & & & \\
Source & DF & Sum of Squares & Mean Square & F Ratio \\
Lack Of Fit & 5 & 0.00183091 & 0.000366 & 0.8028 \\
Pure Error & 40 & 0.01824444 & 0.000456 & Prob $>$ F \\
Total Error & 45 & 0.02007535 & 0.5544 \\
& & & & Max RSq \\
& & & 0.9985
\end{tabular}

Analysis of Variance
Source DF Sum of Squares Mean Square F Ratio

$\begin{array}{cccrr}\text { Source } & \text { DF } & \text { Sum of Squares } & \text { Mean Square } & \text { F Ratio } \\ \text { Model } & 1 & 12.275810 & 12.2758 & 27516.90\end{array}$

$\begin{array}{lrrrrr}\text { Model } & 1 & 12.275810 & 12.2758 & 27516.90 \\ \text { Error } & 45 & 0.020075 & 0.000446 & \text { Prob }>\text { F } \\ \text { C. } & & 12.29585 & & <.000\end{array}$

$\begin{array}{llrrr}\text { Error } & 45 & 0.020075 & 0.000446 & \begin{array}{l}\text { Prob }>\text { F } \\ \text { C. Total }\end{array} \\ & 46 & 12.295885 & <.0001 \text { * }\end{array}$

\section{Parameter Estimates}

$\begin{array}{lrll}\text { Term } & \text { Estimate } & \text { Std Error t Ratio Prob }>|\mathbf{t}|\end{array}$

Inter $1.0241832<0.006174-165.88<.0001 *$
Bivariate Fit of CU By $\ln ($ std wt\%) Meter=System 1, Date=26-Nov, Position=Frwrd

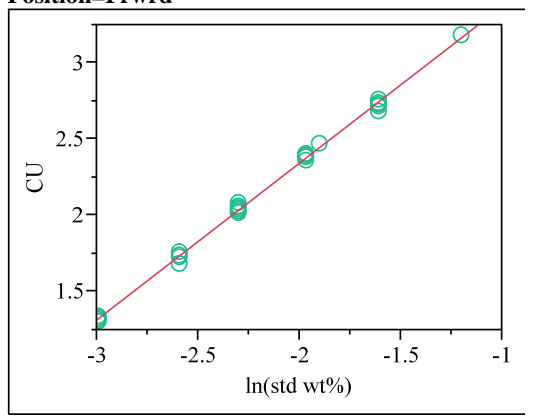

\section{- Linear Fit}

Linear Fit

$\mathrm{CU}=4.4035862+1.0276655^{*} \ln (\mathrm{std} \mathrm{wt} \%)$

Summary of Fit

RSquare

0.998379

RSquare Adj

Root Mean Square Error $\quad 0.021119$

$\begin{array}{lr}\text { Mean of Response } & 2.079787 \\ \text { Observations (or Sum Wgts) } & 47\end{array}$

\section{Lack Of Fi}

Source DF Sum of Squares Mean Square F Ratio $\begin{array}{lllll}\text { Lack Of Fit } & 5 & 0.00420295 & 0.000841 & 2.1191\end{array}$ $\begin{array}{lllll}\text { Pure Error } & 40 & 0.01586667 & 0.000397 & \text { Prob }>\text { F }\end{array}$

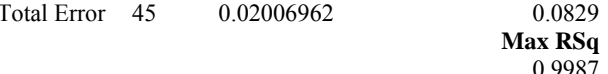

Analysis of Variance

Source DF Sum of Squares Mean Square F Ratio

$\begin{array}{llllll}\text { Model } & 1 & 12.359428 & 12.3594 & 27712.25\end{array}$

$0.020070 \quad 0.000446$ Prob > F

$\begin{array}{lrr}\text { C. Total } 46<12.379498 & <.0001 *\end{array}$

Parameter Estimates

Term Estimate Std Error t Ratio Prob $>|\mathbf{t}|$

$\begin{array}{lrrrr}\text { Intercept } & 4.4035862 & 0.014295 & 308.05<.0001^{*} \\ \text { In(std wt\%) } & 1.0276655 & 0.006173 & 166.47<0001^{*}\end{array}$ 
Exhibit A3. Linear Model of CU Values versus Natural Logarithm of Wt\% Solids Values

Bivariate Fit of CU By ln(std wt\%) Meter=System 1, Date=26-Nov, Position=Left

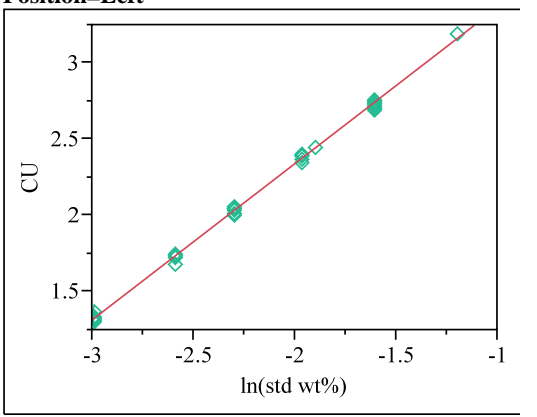

\section{Linear Fit}

Linear Fit

$\mathrm{CU}=4.3933838+1.0242827 * \ln (\mathrm{std} w \mathrm{w} \%)$

\section{Summary of Fit}

RSquare

RSquare Adj $\quad 0.998375$

2.077234

Observations (or Sum Wgts) $\quad 47$

\begin{tabular}{lrrrr}
\multicolumn{2}{l}{ Lack Of Fit } & & & \\
Source & DF & Sum of Squares & Mean Square & F Ratio \\
Lack Of Fit & 5 & 0.00283393 & 0.000567 & 1.3567 \\
Pure Error & 40 & 0.01671111 & 0.000418 & Prob $>$ F \\
Total Error & 45 & 0.01954504 & 0.2610 \\
& & & & Max RSq \\
& & & 0.9986
\end{tabular}

Analysis of Variance

Source DF Sum of Squares Mean Square F Ratio

$\begin{array}{llllll}\text { Model } & 1 & 12.278195 & 12.2782 & 28269.00\end{array}$

$\begin{array}{llll}1 & 0.27955 & 0.000434 & \text { Prob }>\text { F }\end{array}$

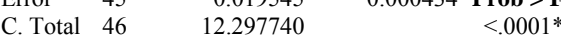

Parameter Estimates

Term Estimate Std Error t Ratio Prob $>|t|$

$\begin{array}{lrrrr}\text { Intercept } & 4.3933838 & 0.014107 & 311.43 & <.0001\end{array}$

$\begin{array}{llllll}\ln \left(\text { std } w \omega^{\circ} \%\right) & 1.0242827 & 0.006092 & 168.13<.0001^{*}\end{array}$
Bivariate Fit of CU By $\ln ($ std wt \%) Meter=System 1, Date=26-Nov, Position=Right

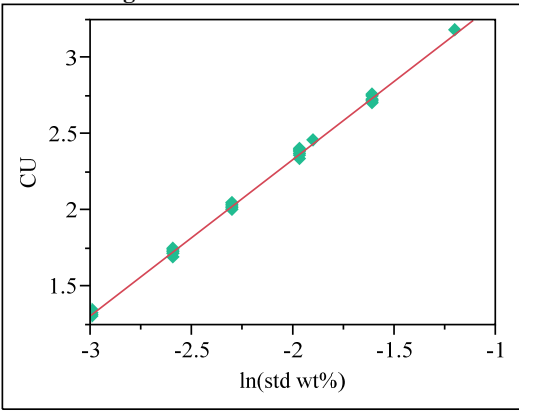

\section{Linear Fit}

Linear Fit

$\mathrm{CU}=4.3916336+1.0254847 * \ln (\mathrm{std} \mathrm{wt} \%)$

\section{Summary of Fit}

RSquare

RSquare Adj

0.998779
0.078086

2.072766

Observations (or Sum Wgts) $\quad 47$

\begin{tabular}{lrrrr}
\multicolumn{2}{l}{ Lack Of Fit } & & & \\
Source & DF & Sum of Squares & Mean Square & F Ratio \\
Lack Of Fit & 5 & 0.00160138 & 0.000320 & 0.9771 \\
Pure Error & 40 & 0.01311111 & 0.000328 & Prob $>$ F \\
Total Error & 45 & 0.01471250 & 0.4435 \\
& & & & Max RSq \\
& & & 0.9989
\end{tabular}

Analysis of Variance
Source DF Sum of Squares Mean Square F Ratio

$\begin{array}{lrrrr}\text { Source } & \text { DF } & \text { Sum of Squares } & \text { Mean Square } & \text { F Ratio } \\ \text { Model } & 1 & 12.307028 & 12.3070 & 37642.58\end{array}$

$\begin{array}{lrrrrr}\text { Model } & 1 & 12.307028 & 12.3070 & 37642.58 \\ \text { Error } & 45 & 0.014712 & 0.000327 & \text { Prob > F }\end{array}$

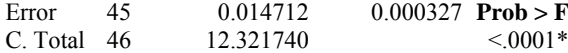

\section{Parameter Estimates}

Term Estimate Std Error t Ratio Prob $>|\mathbf{t}|$

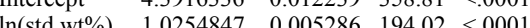

Bivariate Fit of CU By In(std wt \%) Meter=System 1, Date=5-Dec, Position=Back

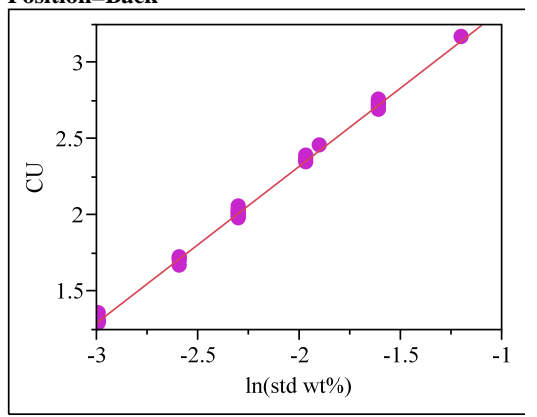

\section{- Linear Fit}

Linear Fit

$\mathrm{CU}=4.3833576+1.0272821 * \ln (\mathrm{std} w \mathrm{wt} \%)$

Summary of Fit

RSquare

$\begin{array}{ll}0.998449 \\ \text { RSquare Adj } & 0.998415\end{array}$

RSquare Adj $\quad 0.998415$

Root Mean Square Eror $\quad 0.020647$

$\begin{array}{lr}\text { Mean of Response } & 2.060426 \\ \text { Observations (or Sum Wgts) } & 47\end{array}$

\section{Lack Of Fi}

Source DF Sum of Squares Mean Square F Ratio $\begin{array}{lllll}\text { Lack Of Fit } & 5 & 0.00333909 & 0.000668 & 1.6859\end{array}$ $\begin{array}{lllll}\text { Pure Error } & 40 & 0.01584444 & 0.000396 & \text { Prob }>\text { F } \\ & & \end{array}$ $\begin{array}{rrrr}\text { Total Error } 45 & 0.01918353 & 0.1603 \\ \text { Max RSq }\end{array}$ 0.9987

Analysis of Variance

Source DF Sum of Squares Mean Square F Ratio $\begin{array}{lrrrr}\text { Model } & 1 & 12.350208 & 12.3502 & 28970.6 \\ & 45 & 0.019184 & 0.000426 & \end{array}$ $\begin{array}{llll}\text { Error } & 45 & 0.019184 & 0.000426 \text { Prob }>\text { F }\end{array}$

\section{Parameter Estimates}

$\begin{array}{llllll}\text { Parameter Estimates } & & & \\ \text { Term } & \text { Estimate } & \text { Std Error } & \text { t Ratio } & \text { Prob }>\mid \mathbf{t} \text { | } \\ \text { Intercept } & 4.3833576 & 0.013976 & 313.63 & <.0001^{*}\end{array}$

$\begin{array}{llllll}\text { Intercept } & 4.3833576 & 0.013976 & 313.63 & <.0001^{*}\end{array}$ 
Exhibit A3. Linear Model of CU Values versus Natural Logarithm of Wt\% Solids Values

Bivariate Fit of CU By ln(std wt\%) Meter=System 1, Date=5-Dec,

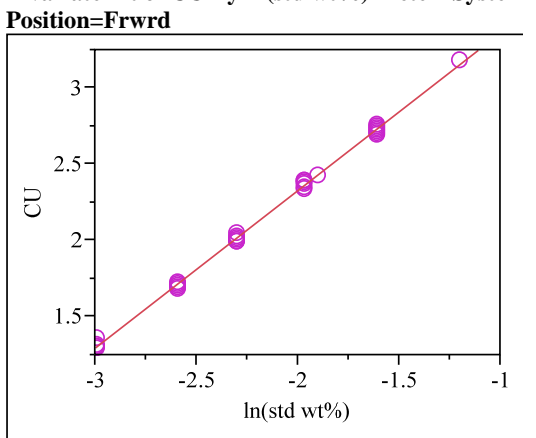

\section{Linear Fit}

Linear Fit

$\mathrm{CU}=4.3981182+1.0328688 * \ln (\mathrm{std} \mathrm{wt} \%)$

\section{Summary of Fit}

RSquare

0.998366

Root Mean Square Error $\quad 0.021077$

$\begin{array}{lr}\text { Mean of Response } & 2.062553 \\ \text { Observations (or Sum Wgts) } & 47\end{array}$

$$
\begin{array}{lrrrr}
\multicolumn{2}{l}{\text { Lack Of Fit }} & & & \\
\text { Source } & \text { DF } & \text { Sum of Squares } & \text { Mean Square } & \text { F Ratio } \\
\text { Lack Of Fit } & 5 & 0.00432380 & 0.000865 & 2.2079 \\
\text { Pure Error } & 40 & 0.01566667 & 0.000392 & \text { Prob }>\text { F } \\
\text { Total Error } & 45 & 0.01999047 & 0.0724 \\
& & & & \text { Max RSq } \\
& & & 0.9987
\end{array}
$$

Analysis of Variance

Source DF Sum of Squares Mean Square F Ratio

$\begin{array}{llllll}\text { Model } & 1 & 12.484903 & 12.4849 & 28104.43\end{array}$

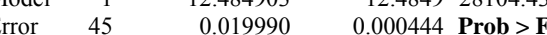

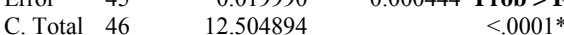

Parameter Estimates

Term Estimate Std Error t Ratio Prob $>|\mathbf{t}|$

$\begin{array}{lllllll}\text { Intercept } & 4.3981182 & 0.014267 & 308.27 & <.0001^{*}\end{array}$
Bivariate Fit of CU By $\ln ($ std wt \%) Meter=System 1, Date=5-Dec, Position=Left

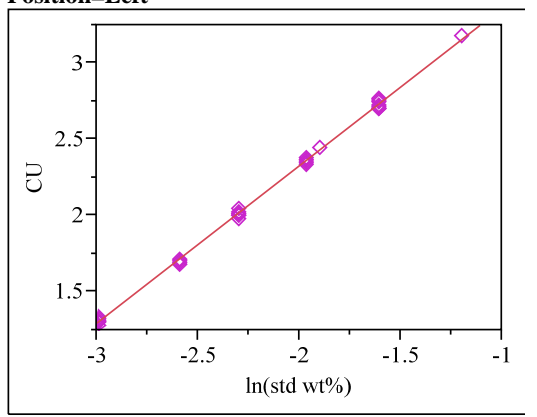

\section{Linear Fit}

Linear Fit

$\mathrm{CU}=4.3954507+1.0327242 * \ln (\mathrm{std} \mathrm{wt} \%)$

\section{Summary of Fit}

RSquare

RSquare Adj

0.998537

2.060213

Observations (or Sum Wgts) $\quad 47$

\section{Lack Of Fit \\ Source DF Sum of Squares Mean Square F Ratio \\ $\begin{array}{lllll}\text { Lack Of Fit } & 5 & 0.00231313 & 0.000463 & 1.1879\end{array}$ \\ $\begin{array}{lllll}\text { Pure Error } 40 & 0.01557778 & 0.000389 & \text { Prob }>\text { F }\end{array}$ \\ $\begin{array}{rrrr}\text { Total Error } & 45 & 0.01789090 & 0.3323 \\ & & & \text { Max RSq }\end{array}$ \\ 0.9988}

Analysis of Variance

Source DF Sum of Squares Mean Square F Ratio

$\begin{array}{lrrrr}\text { Model } & 1 & 12.481407 & 12.4814 & 31393.79\end{array}$

$\begin{array}{lrrrr}\text { Error } & 45 & 0.017891 & 0.000398 \quad \text { Prob }>\text { F }\end{array}$

$\begin{array}{lrr}\text { C. Total } 46 & 12.499298 & 0.000398 \\ & & <.0001^{*}\end{array}$

Parameter Estimates

Term Estimate Std Error t Ratio Prob $>|t|$

$\begin{array}{llllll}\text { Intercept } & 4.3954507 & 0.013497 & 325.66 & <.0001^{*}\end{array}$

$\begin{array}{lllll}\ln \left(\mathrm{std} w \mathrm{wt}^{\circ} \%\right) & 1.0327242 & 0.005829 & 177.18<.0001^{*}\end{array}$
Bivariate Fit of CU By In(std wt \%) Meter=System 1, Date=5-Dec, Position=Right

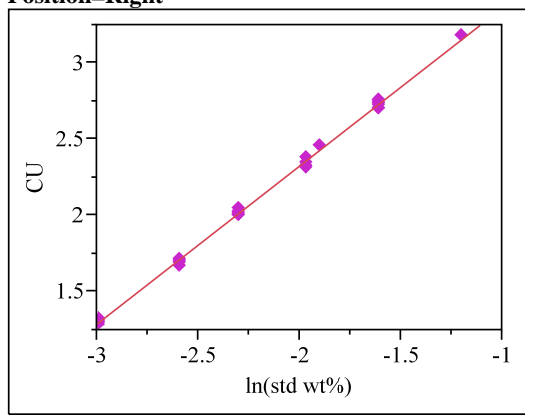

\section{- Linear Fit}

Linear Fit

$\mathrm{CU}=4.39897+1.0355038 * \ln (\mathrm{std} \mathrm{wt} \%)$

Summary of Fit

RSquare

$\begin{array}{ll}0.998567 \\ \text { RSquare Adj } & 0.998535\end{array}$

RSquare Adj

Root Mean Square Eror -0.020005

$\begin{array}{lr}\text { Mean of Response } & 2.057447 \\ \text { Observations (or Sum Wgts) } & 47\end{array}$

\section{Lack Of Fit}

Source DF Sum of Squares Mean Square F Ratio $\begin{array}{lrlll}\text { Lack Of Fit } \quad 5 & 0.00318721 & 0.000637 & 1.7202\end{array}$ $\begin{array}{lllrr}\text { Pure Error } & 40 & 0.01482222 & 0.000371 \quad \text { Prob }>\text { F } \\ \text { Total Error } & 45 & 0.01800943 & 0.1522\end{array}$

$\begin{array}{lrrr}\text { Total Error } 45 \quad 0.01800943 & 0.1522 \\ & & & 0.9988\end{array}$

Analysis of Variance

Source DF Sum of Squares Mean Square F Ratio

$\begin{array}{lrrrr}\text { Model } & 1 & 12.548684 & 12.5487 & 31355.28 \\ & & 0.018009 & 0.0004 & \text { Prob }\end{array}$

$\begin{array}{lrrr} & & 1258009 & 0.0004 \text { Prob }>\text { F }\end{array}$

\section{Parameter Estimates}

Term Estimate Std Error t Ratio Prob $>\mid \mathbf{t}$

$\begin{array}{lrrrrr}\text { Intercept } & 4.39897 & 0.013542 & 324.85 & <.0001^{*}\end{array}$ 
Exhibit A3. Linear Model of CU Values versus Natural Logarithm of Wt\% Solids Values

Bivariate Fit of CU By ln(std wt\%) Meter=System 2, Date=19-Nov, Position=Back

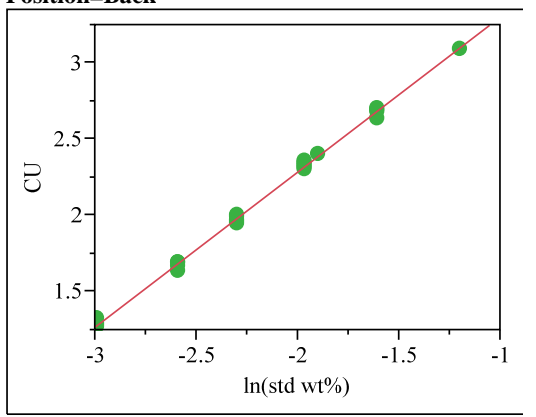

\section{Linear Fit}

Linear Fit

$\mathrm{CU}=4.3228261+1.0166969 * \ln (\mathrm{std} w \mathrm{w} \%)$

\section{Summary of Fit}

RSquare

0.998556

Reot Mean Square Erior 0.01948

$\begin{array}{lr}\text { Mean of Response } & 2.02383 \\ \text { Observations (or Sum Wgts) } & 47\end{array}$

\section{Lack Of Fit}

Source DF Sum of Squares Mean Square F Ratio

$\begin{array}{lllll}\text { Lack Of Fit } & 5 & 0.00292971 & 0.000586 & 1.6531\end{array}$

$\begin{array}{llll}\text { Pure Error } 40 & 0.01417778 & 0.000354 \quad \text { Prob }>\text { F }\end{array}$

$\begin{array}{llr}\text { Total Error } \quad 45 & 0.01710749 & 0.000354 \\ & & \end{array}$

Max RSq

Analysis of Variance
Source DF Sum of Squares Mean Square F Ratio

$\begin{array}{cccrr}\text { Source } & \text { DF } & \text { Sum of Squares } & \text { Mean Square } & \text { F Ratio } \\ \text { Model } & 1 & 12.097003 & 12.0970 & 31820.28\end{array}$

$\begin{array}{lrrrrr}\text { Model } & 1 & 12.097003 & 12.0970 & 31820.28 \\ \text { Error } & 45 & 0.017107 & 0.00038 & \text { Prob }>\text { F }\end{array}$

$\begin{array}{llrr}\text { Error } & 45 & 0.017107 & 0.00038 \underset{\text { Prob }>\text { F }}{<} \\ \text { C. Total } & 46 & 12.114111 & <0001^{*}\end{array}$

\section{Parameter Estimates}

Term Estimate Std Error t Ratio Prob $>\mid t$

$\begin{array}{lrrrr}\text { Intercept } & 4.3228261 & 0.013198 & 327.53 & <.0001^{*}\end{array}$

$\begin{array}{lrrrr}\ln \left(\text { std } w \omega^{\circ} \%\right) & 1.0166969 & 0.0057 & 178.38<.0001^{*}\end{array}$
Bivariate Fit of CU By $\ln ($ std wt \%) Meter=System 2, Date=19-Nov, Position=Frwrd

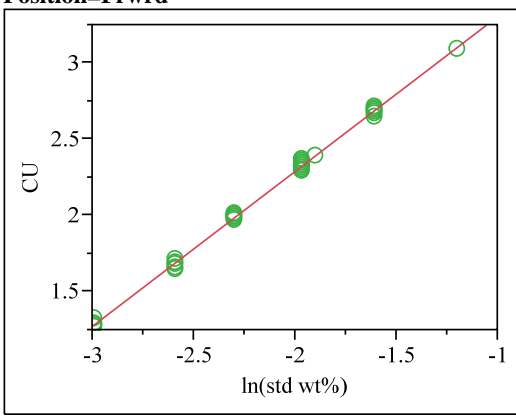

\section{Linear Fit}

Linear Fit

$\mathrm{CU}=4.3260713+1.016062 * \ln (\mathrm{std} \mathrm{w} \mathrm{t} \%)$

\section{Summary of Fit}

RSquare

RSquare Adj

Root Mean Square

Observations (or Sum Wgts) $\quad 47$

\subsection{5}

0.998421 0.02383

$\begin{array}{lrrrr}\begin{array}{l}\text { Lack Of Fit } \\ \text { Source }\end{array} & \text { DF } & \text { Sum of Squares } & \text { Mean Square } & \text { F Ratio } \\ \text { Lack Of Fit } & 5 & 0.00227387 & 0.000455 & 1.1077 \\ \text { Pure Error } & 40 & 0.01642222 & 0.000411 & \begin{array}{r}\text { Prob }>\text { F } \\ \text { Total Error }\end{array} \\ & 45 & 0.01869609 & 0.3716 \\ & & & & \text { Max RSq } \\ & & & 0.9986\end{array}$

Analysis of Variance
Source DF Sum of Squares Mean Square F Ratio $\begin{array}{cccrr}\text { Source } & \text { DF } & \text { Sum of Squares } & \text { Mean Square } & \text { F Ratio } \\ \text { Model } & 1 & 12.081900 & 12.0819 & 29080.17\end{array}$ $\begin{array}{lrrrr}\text { Model } & 1 & 12.081900 & 12.0819 & 29080.17 \\ \text { Error } & 45 & 0.018696 & 0.000415 & \text { Prob }>\text { F }\end{array}$ $\begin{array}{lrrr}\text { Error } & 45 & 0.018696 & 0.000415 \\ \text { C. Total } & 46 & 12.100596 & \begin{array}{l}\text { Prob > } \\ <.0001 *\end{array}\end{array}$

\section{Parameter Estimates}

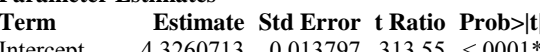

$\begin{array}{llll} & \end{array}$
Bivariate Fit of CU By ln(std wt \%) Meter=System 2, Date=19-Nov, Position=Left

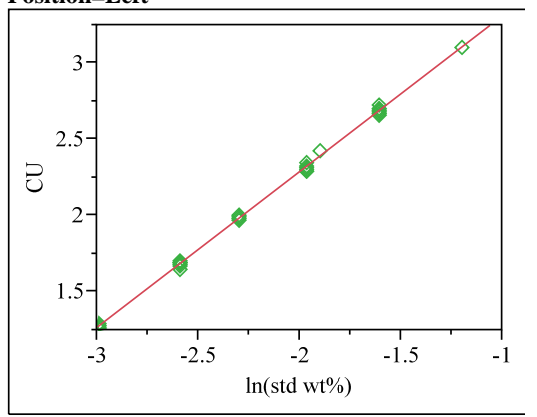

\section{- Linear Fit}

Linear Fit

$\mathrm{CU}=4.3334457+1.0204523 * \ln (\mathrm{std} w \mathrm{w} \%)$

Summary of Fit

RSquare

$\begin{array}{ll}0.998853 \\ \text { RSquare Adj } & 0.998827\end{array}$

Root Mean Square Error $\quad 0.017636$

Root Mean Square Eror $\quad 0.017636$

$\begin{array}{lr}\text { Mean of Response } & 2.025957 \\ \text { Observations (or Sum Wgts) } & 47\end{array}$

\section{Lack Of Fit}

Source DF Sum of Squares Mean Square F Ratio $\begin{array}{lllll}\text { Lack Of Fit } & 5 & 0.00157461 & 0.000315 & 1.0141\end{array}$ $\begin{array}{lrrrr}\text { Pure Error } & 40 & 0.01242222 & 0.000311 \quad \text { Prob }>\text { F }\end{array}$

$\begin{array}{rrrr}\text { Total Error } \quad 45 \quad 0.01399684 & \begin{array}{r}0.4222 \\ \text { Max RSq }\end{array}\end{array}$

Analysis of Variance

Source DF Sum of Squares Mean Square F Ratio

$\begin{array}{lllll}\text { Model } & 1 & 12.186535 & 12.1865 & 39179.86\end{array}$

$\begin{array}{lrrr} & & 12.1835 & 0.000311 \text { Prob > F }\end{array}$

\section{Parameter Estimates}

$\begin{array}{llll}\text { Parameter Estimates } & & \\ \text { Term } & \text { Estimate } & \text { Std Error } & \text { t Ratio Prob }>|\mathbf{t}|\end{array}$

$\begin{array}{lllll}\ln (\mathrm{std} w \mathrm{w} \%) & 1.0204523 & 0.005155 & 197.94<.0001\end{array}$ 
Exhibit A3. Linear Model of CU Values versus Natural Logarithm of Wt\% Solids Values

Bivariate Fit of CU By ln(std wt\%) Meter=System 2, Date=19-Nov, Position=Right

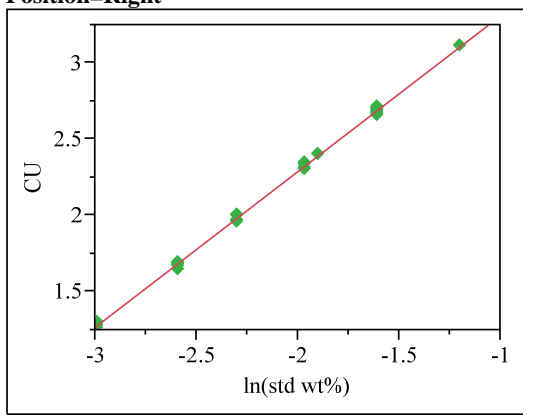

\section{Linear Fit}

Linear Fit

$\mathrm{CU}=4.3335634+1.0206926 * \ln (\mathrm{std} w \mathrm{w} \%)$

\section{Summary of Fit}

RSquare

0.998921

Root

$\begin{array}{lr}\text { Mean of Response } & 2.025532 \\ \text { Observations (or Sum Wgts) } & 47\end{array}$

\section{Lack Of Fit}

Source DF Sum of Squares Mean Square F Ratio

$\begin{array}{lllll}\text { Lack Of Fit } & 5 & 0.00104298 & 0.000209 & 0.7045\end{array}$

$\begin{array}{lllll}\text { Pure Error } 40 & 0.01184444 & 0.000296 & \text { Prob }>\text { F }\end{array}$

$\begin{array}{rrrr}\text { Total Error } & 45 & 0.01288743 & 0.6234 \\ & & \text { Max RSq }\end{array}$

0.9990

Analysis of Variance

Source DF Sum of Squares Mean Square F Ratio

$\begin{array}{lllll}\text { Model } & 1 & 12.192274 & 12.1923 & 42572.68\end{array}$

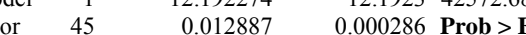

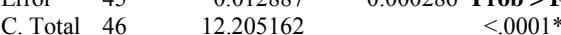

Parameter Estimates

Term Estimate Std Error t Ratio Prob $>|t|$

$\begin{array}{llrrr}\text { Intercept } & 4.3335634 & 0.011455 & 378.31<.0001\end{array}$

$\begin{array}{llllll}\ln \left(\text { std } w \omega^{\circ} \%\right) & 1.0206926 & 0.004947 & 206.33<.0001 *\end{array}$
Bivariate Fit of CU By $\ln ($ std wt \%) Meter=System 2, Date=20-Nov, Position=Back

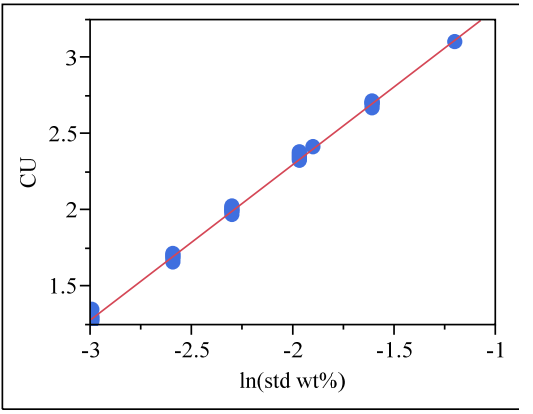

\section{Linear Fit}

Linear Fit

$\mathrm{CU}=4.347277+1.0196061 * \ln (\mathrm{std} \mathrm{wt} \%)$

\section{Summary of Fit}

RSquare

$\begin{array}{ll} & 0.998627 \\ \text { RSquare Adj } & 0.998596\end{array}$

Root Mean Square Error $\quad 0.019282$

Mean of Response 2.041702

Observations (or Sum Wgts) $\quad 47$

$\begin{array}{lrrrr}\begin{array}{l}\text { Lack Of Fit } \\ \text { Source }\end{array} & \text { DF } & \text { Sum of Squares } & \text { Mean Square } & \text { F Ratio } \\ \text { Lack Of Fit } & 5 & 0.00250800 & 0.000502 & 1.4107 \\ \text { Pure Error } & 40 & 0.01422222 & 0.000356 & \begin{array}{l}\text { Prob }>\text { F } \\ \text { Total Error }\end{array} \\ & 45 & 0.01673022 & 0.2412 \\ & & & & \text { Max RSq } \\ & & 0.9988\end{array}$

Analysis of Variance
Source DF Sum of Squares Mean Square F Ratio

$\begin{array}{cccrr}\text { Source } & \text { DF } & \text { Sum of Squares } & \text { Mean Square } & \text { F Ratio } \\ \text { Model } & 1 & 12.166334 & 12.1663 & 32724.32\end{array}$

$\begin{array}{lrrrrr}\text { Model } & 1 & 12.166334 & 12.1663 & 32724.32 \\ \text { Error } & 45 & 0.016730 & 0.000372 & \text { Prob }>\text { F }\end{array}$

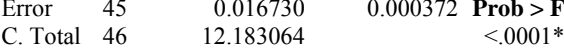

\section{Parameter Estimates}

Term Estimate Std Error t Ratio Prob $>|t|$

$\begin{array}{llrlll}\text { Intercept } & 4.347277 & 0.013052 & 333.08 & <.0001^{*}\end{array}$

$\begin{array}{lllll}\ln (\mathrm{std} \mathrm{wt} \%) & 1.0196061 & 0.005636 & 180.90<0001^{*}\end{array}$
Bivariate Fit of CU By $\ln ($ std wt \%) Meter=System 2, Date=20-Nov, Position=Frwrd

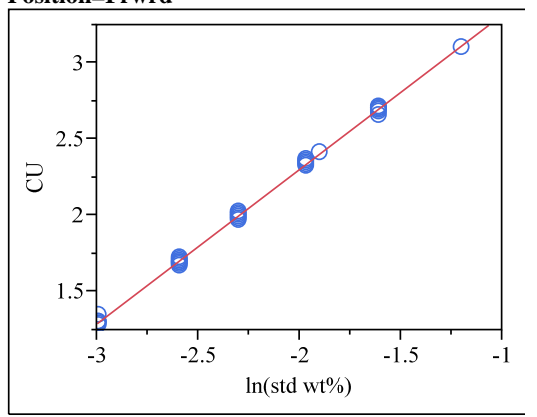

\section{- Linear Fit}

Linear Fit

$\mathrm{CU}=4.3309166+1.0124651 * \ln (\mathrm{std} w \mathrm{w} \%)$

Summary of Fit

RSquare

$\begin{array}{ll}0.998561 \\ \text { RSquare Adj } & 0.998529\end{array}$

0.998529
Root Mean Square Error $\quad 0.019599$

Root Mean Square Eror -0.019599

$\begin{array}{lr}\text { Mean of Response } & 2.041489 \\ \text { Observations (or Sum Wgts) } & 47\end{array}$

\section{Lack Of Fit}

Source DF Sum of Squares Mean Square F Ratio

$\begin{array}{lllll}\text { Lack Of Fit } & 5 & 0.00192932 & 0.000386 & 1.0051\end{array}$

$\begin{array}{lllll}\text { Pure Error } & 40 & 0.01535556 & 0.000384 & \text { Prob }>\text { F }\end{array}$

$\begin{array}{llrr}\text { Total Error } & 45 & 0.01728487 & 0.4273 \\ & & & \text { Max RSq } \\ & 0.9987\end{array}$

Analysis of Variance

Source DF Sum of Squares Mean Square F Ratio

$\begin{array}{lrrrr}\text { Model } & 1 & 11.996511 & 11.9965 & 31232.1 \\ & 45 & 0.017285 & 0.000384 & \end{array}$

$\begin{array}{lrrr}\text { Error } & 45 & 0.017285 & 0.000384 \underset{\text { Prob }>\text { F }}{<.0001 *} \\ \text { C. Total } & 46 & 12.013796 & \end{array}$

\section{Parameter Estimates}

$\begin{array}{lll}\text { Parameter Estimates } & & \\ \text { Term } & \text { Estimate Std Error t Ratio Prob }>|\mathbf{t}|\end{array}$

$\begin{array}{llllll}\text { Intercept } & 4.330166 & 0.013266 & 326.46 & <.0001\end{array}$ 
Exhibit A3. Linear Model of CU Values versus Natural Logarithm of Wt\% Solids Values

Bivariate Fit of CU By $\ln ($ std wt\%) Meter=System 2, Date=20-Nov,

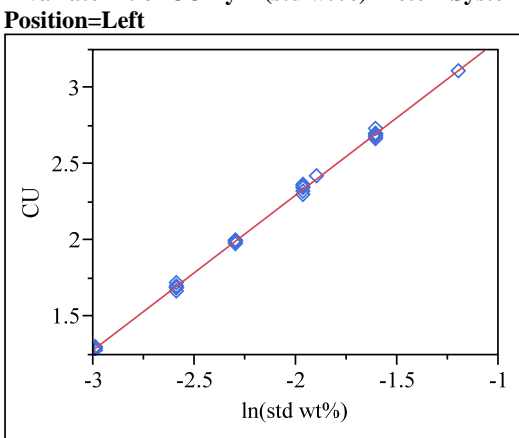

\section{Linear Fit}

Linear Fit

$\mathrm{CU}=4.3367875+1.0165669 * \ln (\mathrm{std} \mathrm{wt} \%)$

\section{Summary of Fit}

RSquare

0.998833
Root Mean Square Error $\quad 0.017523$

Mean of Response 2.038085

Observations (or Sum Wgts) $\quad 47$

\begin{tabular}{lrrrr}
\multicolumn{2}{l}{ Lack Of Fit } & & & \\
Source & DF & Sum of Squares & Mean Square & F Ratio \\
Lack Of Fit & 5 & 0.00070608 & 0.000141 & 0.4308 \\
Pure Error & 40 & 0.01311111 & 0.000328 & Prob $>$ F \\
Total Error & 45 & 0.01381719 & 0.8244 \\
& & & & Max RSq \\
& & & 0.9989
\end{tabular}

Analysis of Variance

Source DF Sum of Squares Mean Square F Ratio

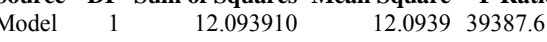

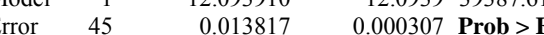

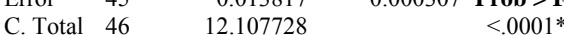

Parameter Estimates

Term Estimate Std Error t Ratio Prob $>\mid t$

$\begin{array}{llrrr}\text { Intercept } & 4.3367875 & 0.011861 & 365.63 & <.0001\end{array}$

$\begin{array}{llllll}\ln \left(\text { std } w \omega^{\circ} \%\right) & 1.0165669 & 0.005122 & 198.46<.0001^{*}\end{array}$
Bivariate Fit of CU By $\ln ($ std wt \%) Meter=System 2, Date=20-Nov, Position=Right

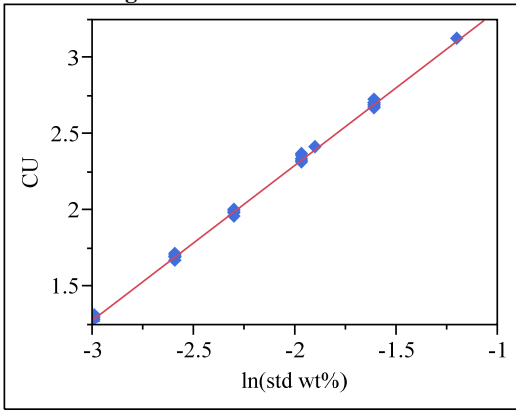

\section{Linear Fit}

Linear Fit

$\mathrm{CU}=4.3378849+1.0175227 * \ln (\mathrm{std} \mathrm{wt} \%)$

\section{Summary of Fit}

RSquare

$\begin{array}{ll} & 0.998902 \\ \text { RSquare Adj } & 0.998877\end{array}$

Root Mean Square Error $\quad 0.017205$

Mean of Response 2.037021

Observations (or Sum Wgts) $\quad 47$

\section{Lack Of Fit \\ Source DF Sum of Squares Mean Square F Ratio \\ $\begin{array}{lllll}\text { Lack Of Fit } & 5 & 0.00098669 & 0.000197 & 0.6400\end{array}$ \\ $\begin{array}{lllll}\text { Pure Error } 40 & 0.01233333 & 0.000308 & \text { Prob }>\text { F }\end{array}$ \\ $\begin{array}{rrrr}\text { Total Error } & 45 & 0.01332002 & 0.6705 \\ & & & \text { Max RSq }\end{array}$ \\ 0.9990}

Analysis of Variance

Source DF Sum of Squares Mean Square F Ratio

$\begin{array}{lllll}\text { Model } & 1 & 12.116663 & 12.1167 & 40934.6\end{array}$

Error $45 \quad 0.013320 \quad 0.000296$ Prob > F

C. Total $46-12.129983$

$<.0001^{*}$

Parameter Estimates

Term Estimate Std Error t Ratio Prob $>|t|$

$\begin{array}{llllll}\text { ntercept } & 4.3378849 & 0.011646 & 372.48 & <.0001 *\end{array}$

$\begin{array}{llllll}\ln (\mathrm{std} w \mathrm{w} \%) & 1.0175227 & 0.005029 & 202.32<.0001^{*}\end{array}$
Bivariate Fit of CU By $\ln ($ std wt \%) Meter=System 2, Date=21-Nov, Position=Back

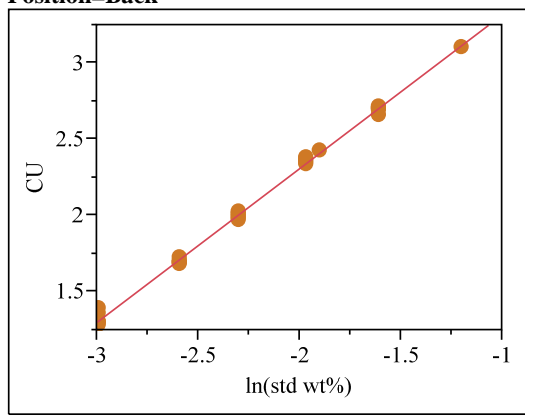

\section{- Linear Fit}

Linear Fit

$\mathrm{CU}=4.3273621+1.0082586^{*} \ln (\mathrm{std} \mathrm{wt} \%)$

Summary of Fit

RSquare

$\begin{array}{ll}0.997948 \\ \text { RSquare Adj } & 0.997903\end{array}$

0.997903
Reot Mean Square Error $\quad 0.023315$

Root Mean Square Eror $\quad 0.023315$

$\begin{array}{lr}\text { Mean of Response } & 2.047447 \\ \text { Observations (or Sum Wgts) } & 47\end{array}$

\section{Lack Of Fi}

Source DF Sum of Squares Mean Square F Ratio

$\begin{array}{lllll}\text { Lack Of Fit } & 5 & 0.00403833 & 0.000808 & 1.5819\end{array}$

$\begin{array}{lllll}\text { Pure Error } & 40 & 0.02042222 & 0.000511 & \text { Prob }>\text { F } \\ & & \end{array}$

$\begin{array}{rrrr}\text { Total Error } & 45 & 0.02446055 & 0.1873 \\ \text { Max RSq }\end{array}$

0.9983

Analysis of Variance

Source DF Sum of Squares Mean Square F Ratio

$\begin{array}{lrrrrr} & \text { Model } & 1 & 11.897033 & 11.8970 & 21886.93\end{array}$

$\begin{array}{lrrr} & & 11.024461 & 0.000544 \text { Prob }>\text { F }\end{array}$

Parameter Estimates

Term Estimate Std Error t Ratio Prob $>\mid \mathbf{t}$

$\begin{array}{llrlll}\text { Intercept } & 4.3273621 & 0.015782 & 274.20< & <.0001^{*} \\ \text { In(std wt\%) } & 1.0082586 & 0.006815 & 147.94<0001^{*}\end{array}$ 
Exhibit A3. Linear Model of CU Values versus Natural Logarithm of Wt\% Solids Values

Bivariate Fit of CU By ln(std wt\%) Meter=System 2, Date=21-Nov,

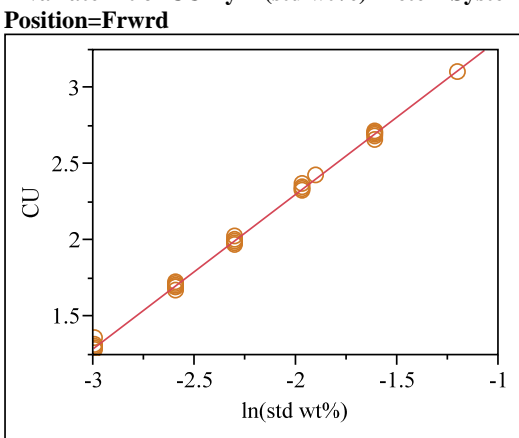

\section{Linear Fit}

Linear Fit

$\mathrm{CU}=4.3356997+1.0133572 * \ln (\mathrm{std} \mathrm{wt} \%)$

\section{Summary of Fit}

RSquare

$\begin{array}{ll} & 0.998364 \\ \text { RSquare Adj } & 0.998328\end{array}$

Root Mean Square Error $\quad 0.020917$

Mean of Response 2.044255

Observations (or Sum Wgts) $\quad 47$

\begin{tabular}{lrrrr}
\multicolumn{2}{l}{ Lack Of Fit } & & & \\
Source & DF & Sum of Squares & Mean Square & F Ratio \\
Lack Of Fit & 5 & 0.00242227 & 0.000484 & 1.1223 \\
Pure Error & 40 & 0.01726667 & 0.000432 & Prob $>$ F \\
Total Error & 45 & 0.01968894 & 0.3642 \\
& & & & Max RSq \\
& & & 0.9986
\end{tabular}

Analysis of Variance

Source DF Sum of Squares Mean Square F Ratio

$\begin{array}{lllll}\text { Model } & 1 & 12.017660 & 12.0177 & 27466.93\end{array}$

$\begin{array}{lllll} & & 0.019689 & 0.000438 \text { Prob }>\text { F }\end{array}$

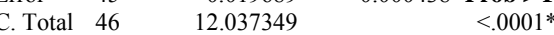

Parameter Estimates

$\begin{array}{lrrrrr}\text { Term } & \text { Estimate } & \text { Std Error } & \text { t Ratio } & \text { Prob }>|\mathbf{t}| \\ \text { Intercept } & 4.3356997 & 0.014159 & 306.22 & <.0001^{*}\end{array}$

$\begin{array}{llllll}\ln \left(\text { std } w \omega^{\circ} \%\right) & 1.0133572 & 0.006114 & 165.73<.0001 *\end{array}$
Bivariate Fit of CU By $\ln ($ std wt \%) Meter=System 2, Date=21-Nov, Position=Left

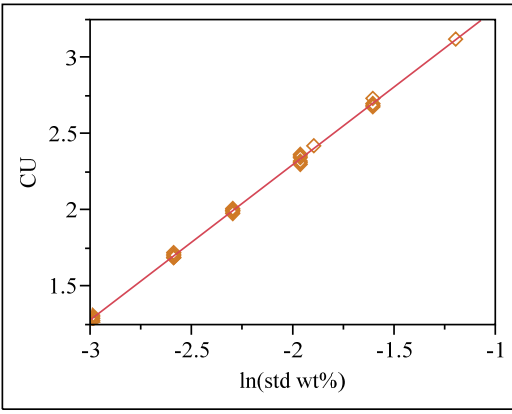

\section{Linear Fit}

Linear Fit

$\mathrm{CU}=4.3448193+1.0184252 * \ln (\mathrm{std} \mathrm{wt} \%)$

\section{Summary of Fit}

RSquare

RSquare Adj

Root Mean Square

0.998983

0.99896 2.041915

Observations (or Sum Wgts)

47

\section{Lack Of Fi}

Source DF Sum of Squares Mean Square F Ratio

$\begin{array}{lllll}\text { Lack Of Fit } & 5 & 0.00069434 & 0.000139 & 0.4761\end{array}$

$\begin{array}{lllll}\text { Pure Error } 40 & 0.01166667 & 0.000292 & \text { Prob }>\text { F }\end{array}$

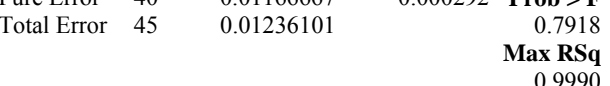

Analysis of Variance

Source DF Sum of Squares Mean Square F Ratio

$\begin{array}{lllll}\text { Model } & 1 & 12.138167 & 12.1382 & 44188.75\end{array}$

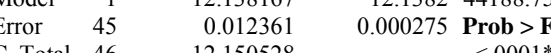

\section{Parameter Estimates}

Term Estimate Std Error t Ratio Prob $>|\mathbf{t}|$

lnters $\quad 1.0184252 \quad 0.004845 \quad 210.21<0001^{*}$
Bivariate Fit of CU By $\ln ($ std wt \%) Meter=System 2, Date=21-Nov, Position=Right

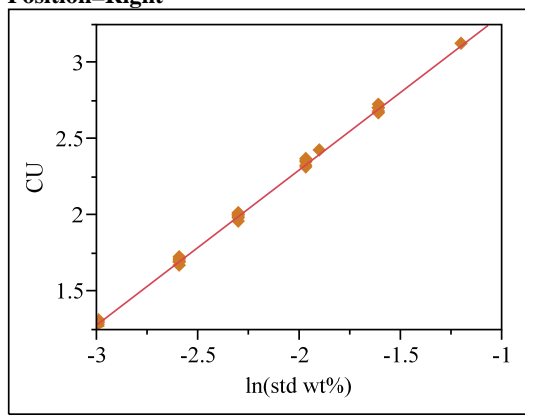

\section{- Linear Fit}

Linear Fit

$\mathrm{CU}=4.3403186+1.0175639 * \ln (\mathrm{std} \mathrm{wt} \%)$

Summary of Fit

RSquare

$\begin{array}{ll}0.99881 \\ \text { RSquare Adj } & 0.998784\end{array}$

Root Mean Square Error $\quad 0.998784$
0.017911

$\begin{array}{ll}\text { Root Mean Square Error } & 0.017911 \\ \text { Mean of Response } & 2.039362\end{array}$

Observations (or Sum Wgts) $\quad 47$

\begin{tabular}{lrrrr}
\multicolumn{2}{l}{ Lack Of Fit } & & & \\
Source & DF & Sum of Squares & Mean Square & F Ratio \\
Lack Of Fit & 5 & 0.00108028 & 0.000216 & 0.6471 \\
Pure Error & 40 & 0.01335556 & 0.000334 & Prob $>$ F \\
Total Error & 45 & 0.01443583 & 0.6653 \\
& & & & Max RSq \\
& & & 0.9989
\end{tabular}

Analysis of Variance

Source DF Sum of Squares Mean Square F Ratio

$\begin{array}{lllll}\text { Model } & 1 & 12.117645 & 12.1176 & 37773.65\end{array}$

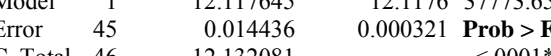

Parameter Estimates

$\begin{array}{lllll}\text { Param } & \text { Estimate } & \text { Std Error } & \text { t Ratio Prob }>|\mathbf{t}| \\ \text { Term } & \end{array}$

$\begin{array}{llllll}\text { Intercept } & 4.3403186 & 0.012124 & 358.00 & <.0001^{*} \\ \ln (\mathrm{std} w \mathrm{wt} \%) & 1.0175639 & 0.005236 & 194.35<.0001^{*}\end{array}$ 
Exhibit A3. Linear Model of CU Values versus Natural Logarithm of Wt\% Solids Values

Bivariate Fit of CU By ln(std wt\%) Meter=System 2, Date=26-Nov, Position=Back

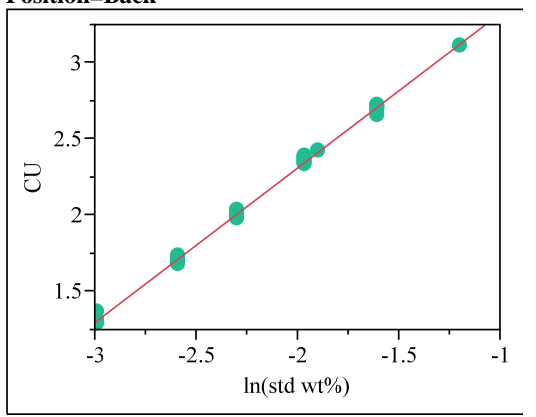

\section{Linear Fit}

Linear Fit

$\mathrm{CU}=4.3431076+1.0126813 * \ln (\operatorname{std} \mathrm{wt} \%)$

\section{Summary of Fit}

RSquare

0.998351

Root Mean Square Eror -0.020756

$\begin{array}{lr}\text { Mean of Response } & 2.053191 \\ \text { Observations (or Sum Wgts) } & 47\end{array}$

$$
\begin{array}{lrrrr}
\begin{array}{l}
\text { Lack Of Fit } \\
\text { Source }
\end{array} & \text { DF } & \text { Sum of Squares } & \text { Mean Square } & \text { F Ratio } \\
\text { Lack Of Fit } & 5 & 0.00343136 & 0.000686 & 1.7205 \\
\text { Pure Error } & 40 & 0.01595556 & 0.000399 & \begin{array}{r}
\text { Prob }>\text { F } \\
\text { Total Error }
\end{array} \\
& 45 & 0.01938692 & 0.1522 \\
& & & & \text { Max RSq } \\
& & & 0.9987
\end{array}
$$

Analysis of Variance

Source DF Sum of Squares Mean Square F Ratio

$\begin{array}{llllll}\text { Model } & 1 & 12.001634 & 12.0016 & 27857.63\end{array}$

$\begin{array}{lllll} & 15 & 0.019387 & 0.000431 & \text { Prob }>\text { F }\end{array}$

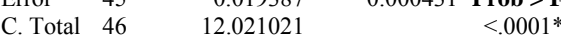

Parameter Estimates

Term Estimate Std Error t Ratio Prob $>|\mathbf{t}|$

$\begin{array}{lll} & \end{array}$
Bivariate Fit of CU By $\ln ($ std wt \%) Meter=System 2, Date=26-Nov, Position=Frwrd

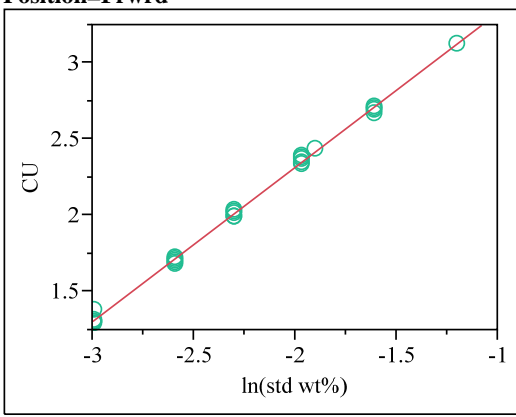

\section{Linear Fit}

Linear Fit

$\mathrm{CU}=4.3446718+1.0119616 * \ln (\mathrm{std} \mathrm{wt} \%)$

\section{Summary of Fit}

RSquare

RSquare Adj

0.998356

Mean of Response $\quad 0.020711$

Observations (or Sum Wgts)

\section{Lack Of Fit \\ Source DF Sum of Squares Mean Square F Ratio $\begin{array}{lllll}\text { Lack Of Fit } & 5 & 0.00290192 & 0.000580 & 1.4156\end{array}$ $\begin{array}{llll}\text { Pure Error } 40 & 0.01640000 & 0.000410 \quad \text { Prob }>\text { F }\end{array}$ $\begin{array}{lll} & 0.01630192\end{array}$ 0.2395
Max RSq}

Analysis of Variance

Source DF Sum of Squares Mean Square F Ratio

$\begin{array}{llllll}\text { Model } & 1 & 11.984583 & 11.9846 & 27940.55\end{array}$

$\begin{array}{lrrrr}\text { Error } & 45 & 0.019302 & 0.000429 & \text { Prob }>\text { F }\end{array}$

\begin{tabular}{lrrr} 
C. Total & 46 & 12.003885 & 0.000429 \\
\hline
\end{tabular}

Parameter Estimates

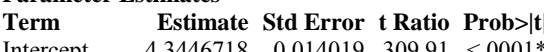

$\begin{array}{llllll} & & \end{array}$
Bivariate Fit of CU By ln(std wt\%) Meter=System 2, Date=26-Nov, Position=Left

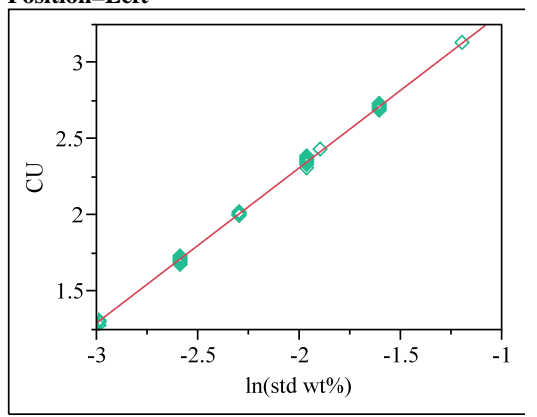

\section{- Linear Fit}

Linear Fit

$\mathrm{CU}=4.3527036+1.0168309^{*} \ln (\mathrm{std} \mathrm{wt} \%)$

Summary of Fit

RSquare

$\begin{array}{lr} & 0.99883 \\ \text { Root Mean Square Error } \quad 0.017552\end{array}$

Mean of Response $\quad 2.053404$

Observations (or Sum Wgts) $\quad 47$

\begin{tabular}{lrrrr}
\multicolumn{2}{l}{ Lack Of Fit } & & & \\
Source & DF & Sum of Squares & Mean Square & F Ratio \\
Lack Of Fit & 5 & 0.00068484 & 0.000137 & 0.4158 \\
Pure Error & 40 & 0.01317778 & 0.000329 & $\begin{array}{r}\text { Prob }>\text { F } \\
\text { Total Error }\end{array}$ \\
& 45 & 0.01386262 & 0.8350 \\
& & & & Max RSq \\
& & & 0.9989
\end{tabular}

Analysis of Variance

Source DF Sum of Squares Mean Square F Ratio

$\begin{array}{lllll}\text { Model } & 1 & 12.100193 & 12.1002 & 39278.91\end{array}$

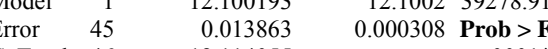

$\begin{array}{lll}\text { C. Total } 46 & 12.114055 & 0.00308 \\ & <.0001^{*}\end{array}$

Parameter Estimates

Term Estimate Std Error t Ratio Prob $>\mid \mathbf{t}$

$\begin{array}{lrrrrr}\text { Intercept } & 4.3527036 & 0.011881 & 366.37 & <.0001^{*} \\ \ln (\mathrm{std} w \mathrm{wt} \%) & 1.0168309 & 0.005131 & 198.19<0001^{*}\end{array}$ 
Exhibit A3. Linear Model of CU Values versus Natural Logarithm of Wt\% Solids Values

Bivariate Fit of CU By ln(std wt\%) Meter=System 2, Date=26-Nov, Position=Right

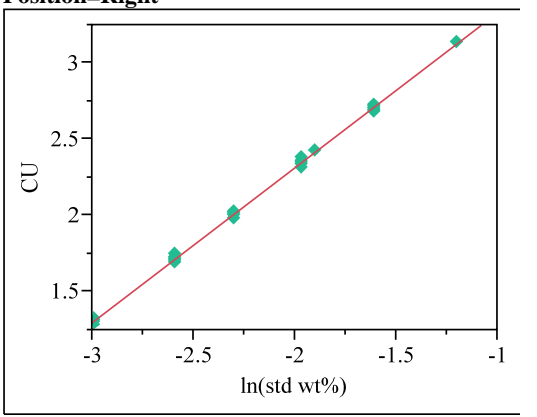

\section{Linear Fit}

Linear Fit

$\mathrm{CU}=4.3476613+1.0152596 * \ln (\mathrm{std} \mathrm{wt} \%)$

\section{Summary of Fit}

RSquare

RSquare Adj

0.017193

Observations (or Sum Wgts) $\quad 47$

$\begin{array}{lrrrr}\begin{array}{l}\text { Lack Of Fit } \\ \text { Source }\end{array} & \text { DF } & \text { Sum of Squares } & \text { Mean Square } & \text { F Ratio } \\ \text { Lack Of Fit } & 5 & 0.00059035 & 0.000118 & 0.3715 \\ \text { Pure Error } & 40 & 0.01271111 & 0.000318 & \begin{array}{r}\text { Prob }>\text { F } \\ \text { Total Error }\end{array} \\ & 45 & 0.01330146 & 0.8651 \\ & & & & \text { Max RSq } \\ & & & 0.9989\end{array}$

Analysis of Variance

Source DF Sum of Squares Mean Square F Ratio

$\begin{array}{lllll}\text { Model } & 1 & 12.062826 & 12.0628 & 40809.59\end{array}$

$\begin{array}{lllll} & & 12.062826 & 12.0628 & 40809.59 \\ \text { Error } & 45 & 0.013301 & 0.000296 & \text { Prob }>\text { F }\end{array}$

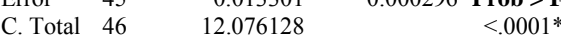

Parameter Estimates

Term Estimate Std Error t Ratio Prob $>|t|$

$\begin{array}{llrrr}\text { Intercept } & 4.3476613 & 0.011638 & 373.58<.0001^{*}\end{array}$

$\begin{array}{llllll}\ln \left(\text { std } w \omega^{\circ} \%\right) & 1.0152596 & 0.005026 & 202.01<.0001 *\end{array}$
Bivariate Fit of CU By $\ln ($ std wt \%) Meter=System 2, Date=27-Nov, Position=Back

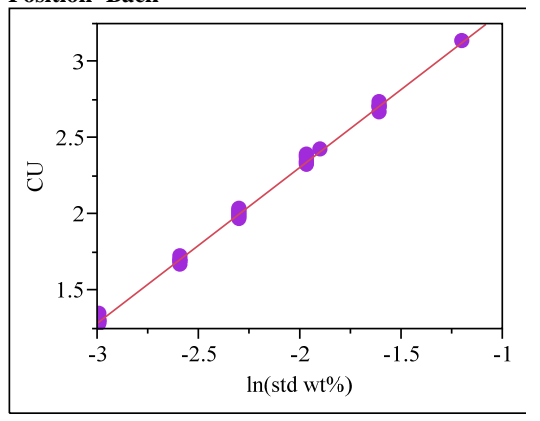

\section{Linear Fit}

Linear Fit

$\mathrm{CU}=4.3599747+1.0222105 * \ln (\mathrm{std} \mathrm{wt} \%)$

\section{Summary of Fit}

RSquare

RSquare Adj

\begin{tabular}{ll}
0.998562 \\
\hline
\end{tabular}

Mean of Reso

Observations (or Sum Wgts) $\quad 47$

\begin{tabular}{lrrrr}
\multicolumn{2}{l}{ Lack Of Fit } & & & \\
Source & DF & Sum of Squares & Mean Square & F Ratio \\
Lack Of Fit & 5 & 0.00167408 & 0.000335 & 0.8610 \\
Pure Error & 40 & 0.01555556 & 0.000389 & $\begin{array}{r}\text { Prob }>\text { F } \\
\text { Total Error }\end{array}$ \\
& 45 & 0.01722963 & 0.5156 \\
& & & & $\begin{array}{r}\text { Max RSq } \\
\text { RST } \\
\end{array}$ \\
& & & & 0.9987
\end{tabular}

Analysis of Variance
Source DF Sum of Squares Mean Square F Ratio

$\begin{array}{lrrrr}\text { Source } & \text { DF } & \text { Sum of Squares } & \text { Mean Square } & \text { F Ratio } \\ \text { Model } & 1 & 12.228566 & 12.2286 & 31938.31\end{array}$

$\begin{array}{lrrrrr}\text { Model } & 1 & 12.228566 & 12.2286 & 31938.31 \\ \text { Error } & 45 & 0.017230 & 0.000383 & \text { Prob }>\text { F }\end{array}$

\begin{tabular}{lrrrr} 
Error & 45 & 0.017230 & 0.000383 & $\begin{array}{l}\text { Prob }>\text { F } \\
\text { C. Total }\end{array}$ \\
\hline & 46 & 12.245796 & $<.0001$ *
\end{tabular}

\section{Parameter Estimates}

Term Estimate Std Error t Ratio Prob $>|t|$

$\begin{array}{lrrrr}\text { Intercept } & 4.3599747 & 0.013245 & 329.18<.0001 *\end{array}$

$\begin{array}{llrrr} & \text { In }\left(\text { std } w t^{\circ} \%\right) & 1.0222105 & 0.00572 & 178.71<.0001^{*}\end{array}$
Bivariate Fit of CU By ln(std wt\%) Meter=System 2, Date=27-Nov, Position=Frwrd

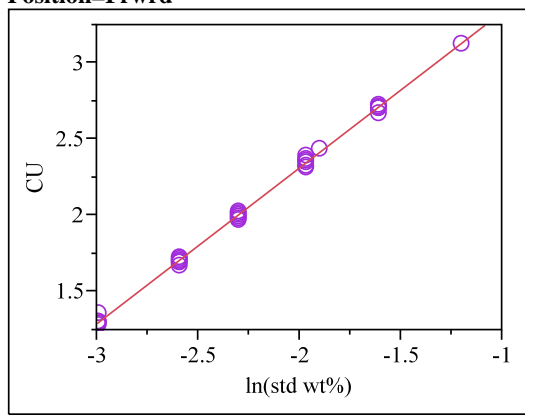

\section{- Linear Fit}

Linear Fit

$\mathrm{CU}=4.3602092+1.0216556^{*} \ln (\mathrm{std} \mathrm{wt} \%)$

Summary of Fit

$\begin{array}{ll}\text { RSquare } & 0.998275 \\ \text { RSquare Adj } & 0.998237\end{array}$

RSquare Adj $\quad 0.998237$

Mean of Response $\quad 2.05$

$\begin{array}{lr}\text { Mean of Response } & 2.05 \\ \text { Observations (or Sum Wgts) } & 47\end{array}$

\section{Lack Of Fit}

Source DF Sum of Squares Mean Square F Ratio $\begin{array}{lrrrr}\text { Lack Of Fit } & 5 & 0.00130700 & 0.000261 & 0.5281\end{array}$ $\begin{array}{lllll}\text { Pure Error } 40 & 0.01980000 & 0.000495 & \text { Prob }>\text { F } \\ & 45 & \end{array}$

$\begin{array}{llr}\text { Total Error } 45 \quad 0.02110700 & \begin{array}{r}0.7536 \\ \text { Max RSq }\end{array} \\ & & \end{array}$

Analysis of Variance

Source DF Sum of Squares Mean Square F Ratio

$\begin{array}{lllll}\text { Model } & 1 & 12.215293 & 12.2153 & 26042.93\end{array}$

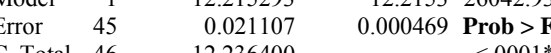

\section{Parameter Estimates}

Term Estimate Std Error t Ratio Prob $>\mid \mathbf{t}$

$\begin{array}{lrrrr}\text { Intercept } & 4.3602092 & 0.01466 & 297.42< & <.0001^{*} \\ \ln (\text { std } \mathrm{wt} \%) & 1.0216556 & 0.006331 & 161.38<.0001^{*}\end{array}$ 
Exhibit A3. Linear Model of CU Values versus Natural Logarithm of Wt\% Solids Values

Bivariate Fit of CU By ln(std wt\%) Meter=System 2, Date=27-Nov, Position=Left

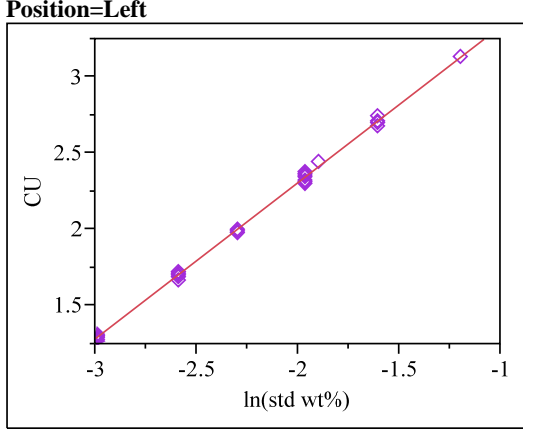

\section{Linear Fit}

Linear Fit

$\mathrm{CU}=4.3595347+1.0239919 * \ln (\mathrm{std} \mathrm{wt} \%)$

\section{Summary of Fit}

RSquare

RSquare Adj

Root Men Square Error

0.998592

2.044043

\section{Lack Of Fit}

Source DF Sum of Squares Mean Square F Ratio

$\begin{array}{lllll}\text { Lack Of Fit } & 5 & 0.00159677 & 0.000319 & 0.8131\end{array}$

$\begin{array}{lrrrr}\text { Pure Error } & 40 & 0.01571111 & 0.000393 & \text { Prob }>\text { F } \\ & 45 & 0.01730789 & & 0.5474\end{array}$

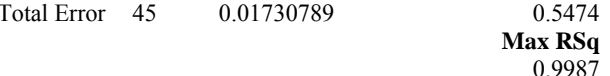

Analysis of Variance

Source DF Sum of Squares Mean Square F Ratio

$\begin{array}{lllll}\text { Model } & 1 & 12.271224 & 12.2712 & 31904.83\end{array}$

$0.017308-0.000385$ Prob $>$ F

Parameter Estimates

Term $\quad$ Estimate Std Error t Ratio Prob $>|t|$

Term Estimate Std Error t Ratio Prob $>\mid$

$\begin{array}{llllll} & \text { Estercept } & 4.3595347 & 0.013275 & 328.40<.0001^{*}\end{array}$
Bivariate Fit of CU By $\ln ($ std wt \%) Meter=System 2, Date=27-Nov, Position=Right

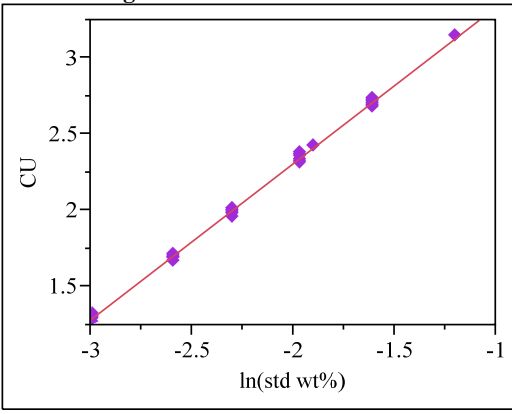

\section{Linear Fit}

Linear Fit

$\mathrm{CU}=4.3605374+1.0248117 * \ln (\mathrm{std} \mathrm{wt} \%)$

\section{Summary of Fit}

RSquare

RSquare Adj

Root Mean Square

0.998689

0.998659 2.043191

Observations (or Sum Wgts) $\quad 47$

\begin{tabular}{lrrrr}
\multicolumn{2}{l}{ Lack Of Fit } & & & \\
Source & DF & Sum of Squares & Mean Square & F Ratio \\
Lack Of Fit & 5 & 0.00162928 & 0.000326 & 0.8982 \\
Pure Error & 40 & 0.0145111 & 0.000363 & Prob $>$ F \\
Total Error & 45 & 0.01614039 & 0.4917 \\
& & & & Max RSq \\
& & & 0.9988
\end{tabular}

Analysis of Variance
Source DF Sum of Squares Mean Square F Ratio

$\begin{array}{crrrr}\text { Source } & \text { DF } & \text { Sum of Squares } & \text { Mean Square } & \text { F Ratio } \\ \text { Model } & 1 & 12.290881 & 12.2909 & 34267.43\end{array}$

$\begin{array}{lrrrrr}\text { Model } & 1 & 12.290881 & 12.2909 & 34267.43 \\ \text { Error } & 45 & 0.016140 & 0.000359 & \text { Prob }>\text { F }\end{array}$

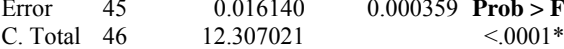

\section{Parameter Estimates}

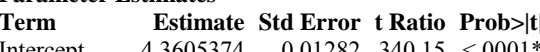

$\begin{array}{lllll} & 0.01282 & 340.15<.0001 *\end{array}$
Bivariate Fit of CU By In(std wt \%) Meter=System 2, Date=3-Dec, Position=Back

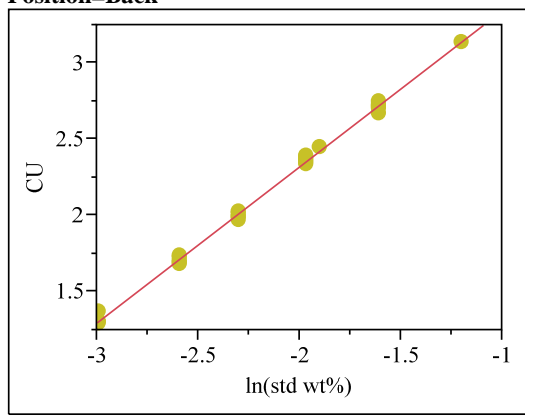

\section{- Linear Fit}

Linear Fit

$\mathrm{CU}=4.3690375+1.0231134 * \ln (\mathrm{std} \mathrm{wt} \%)$

Summary of Fit

RSquare

$\begin{array}{ll}0.998388 \\ \text { RSquare Adj } & 0.998352\end{array}$

Root Mean Square Error $\quad 0.020968$

Root Mean Square Eroor $\quad 0.020968$

$\begin{array}{lr}\text { Mean of Response } & 2.055532 \\ \text { Observations (or Sum Wgts) } & 47\end{array}$

Source DF Sum of Squares Mean Square F Ratio $\begin{array}{lllll}\text { Lack Of Fit } & 5 & 0.00222902 & 0.000446 & 1.0158\end{array}$ $\begin{array}{lllll}\text { Pure Error } & 40 & 0.01755556 & 0.000439 & \text { Prob }>\text { F } \\ \text { Total Error } & 45 & 0.01978458 & & 0.4212\end{array}$

$\begin{array}{lrrr}\text { Total Error } \quad 45 \quad 0.01978458 & 0.4212 \\ & & & 0.9986\end{array}$

Analysis of Variance

Source DF Sum of Squares Mean Square F Ratio

$\begin{array}{lllll}\text { Model } & 1 & 12.250177 & 12.2502 & 27863.02\end{array}$

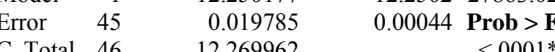

\section{Parameter Estimates}

Term Estimate Std Error t Ratio Prob $>\mid \mathbf{t}$

$\begin{array}{lrrrrr}\text { Intercept } & 4.3690375 & 0.014193 & 307.83 & <.0001^{*} \\ \text { In(std wt\%) } & 1.0231134 & 0.006129 & 166.92<.0001^{*}\end{array}$ 
Exhibit A3. Linear Model of CU Values versus Natural Logarithm of $\mathrm{Wt} \%$ Solids Values

Bivariate Fit of CU By ln(std wt \%) Meter=System 2, Date=3-Dec,

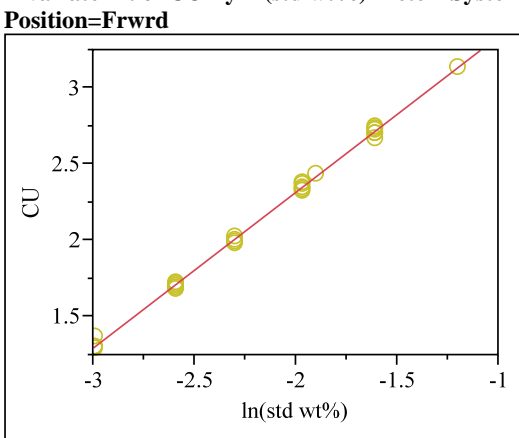

\section{Linear Fit}

Linear Fit

$\mathrm{CU}=4.3638079+1.0217416 * \ln (\mathrm{std} w \mathrm{w} \%)$

\section{Summary of Fit}

RSquare

RSquare Adj

0.998504

(O)

Observations (or Sum Wgts) $\quad 47$

\begin{tabular}{lrrrr}
\multicolumn{2}{l}{ Lack Of Fit } & & & \\
Source & DF & Sum of Squares & Mean Square & F Ratio \\
Lack Of Fit & 5 & 0.00121718 & 0.000243 & 0.5835 \\
Pure Error & 40 & 0.01668889 & 0.000417 & Prob $>$ F \\
Total Error & 45 & 0.01790607 & 0.7124 \\
& & & & Max RSq \\
& & & 0.9986
\end{tabular}

Analysis of Variance

Source DF Sum of Squares Mean Square F Ratio

$\begin{array}{lllll}\text { Model } & 1 & 12.217349 & 12.2173 & 30703.59\end{array}$

$\begin{array}{llll}1 & 12.217349 & 0.000398 & \text { Prob }>\text { F }\end{array}$

$\begin{array}{lrrr}\text { C. Total } & 46 & 12.235255 & <.00391^{*}\end{array}$

Parameter Estimates

Term Estimate Std Error t Ratio Prob $>\mid t$

$\begin{array}{llllll}\text { Intercept } & 4.3638079 & 0.013503 & 323.18 & <.0001\end{array}$

$\begin{array}{llllll} & \text { In(std wt\%) } & 4.3638079 & 0.013503 & 323.18<.0001^{*}\end{array}$
Bivariate Fit of CU By $\ln ($ std wt \%) Meter=System 2, Date=3-Dec, Position=Left

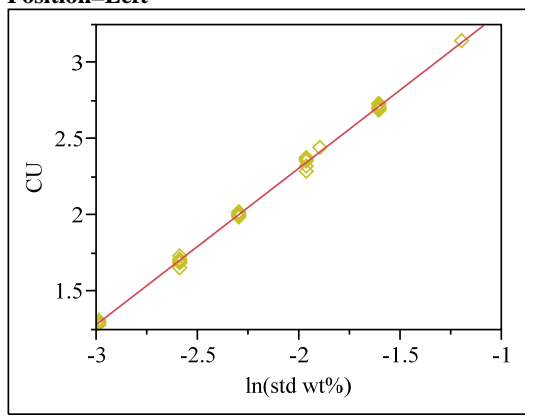

\section{Linear Fit}

Linear Fit

$\mathrm{CU}=4.3684259+1.0257598 * \ln (\mathrm{std} \mathrm{wt} \%)$

\section{Summary of Fit}

RSquare

RSquare Adj

\begin{tabular}{ll}
0.998507 \\
\hline
\end{tabular}

2.048936

Observations (or Sum Wgts) $\quad 47$

\begin{tabular}{lrrrr}
\multicolumn{2}{l}{ Lack Of Fit } & & & \\
Source & DF & Sum of Squares & Mean Square & F Ratio \\
Lack Of Fit & 5 & 0.00150347 & 0.000301 & 0.7285 \\
Pure Error & 40 & 0.0165111 & 0.000413 & Prob $>$ F \\
Total Error & 45 & 0.01801458 & 0.6062 \\
& & & & Max RSq \\
& & & 0.9987
\end{tabular}

Analysis of Variance
Source DF Sum of Squares Mean Square F Ratio

$\begin{array}{ccrrr}\text { Source } & \text { DF } & \text { Sum of Squares } & \text { Mean Square } & \text { F Ratio } \\ \text { Model } & 1 & 12.313632 & 12.3136 & 30759.16\end{array}$

$\begin{array}{lrrrr}\text { Model } & 1 & 12.313632 & 12.3136 & 30759.16 \\ \text { Error } & 45 & 0.018015 & 0.0004 & \text { Prob }>\text { F } \\ \text { C. } & & 12.33647 & & <000\end{array}$

$\begin{array}{lrrr}\text { Error } & 45 & 0.018015 & 0.0004 \underset{\text { Prob }>\text { F }}{<} \\ \text { C. Total } & 46 & 12.331647 & <.0001^{*}\end{array}$

\section{Parameter Estimates}

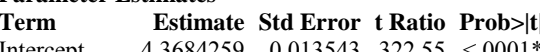

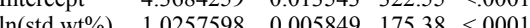

Bivariate Fit of CU By In(std wt \%) Meter=System 2, Date=3-Dec, Position=Right

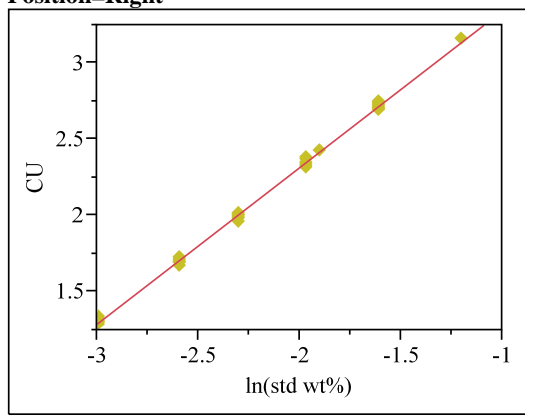

\section{Linear Fit}

Linear Fit

$\mathrm{CU}=4.3704702+1.0265697 * \ln (\mathrm{std} w \mathrm{wt} \%)$

Summary of Fit

RSquare

0.998407

Root Mean Square Error $\quad 0.998371$

Root Mean Square Eror -0.02013

$\begin{array}{lr}\text { Mean of Response } & 2.049149 \\ \text { Observations (or Sum Wgts) } & 47\end{array}$

\section{Lack Of Fit}

Source DF Sum of Squares Mean Square F Ratio $\begin{array}{lllll}\text { Lack Of Fit } & 5 & 0.00203588 & 0.000407 & 0.9231\end{array}$ $\begin{array}{lll}\text { Pure Error } & 40 & 0.01764444\end{array}$ $\begin{array}{llrr}\text { Total Error } 45 & 0.01968032 & 0.4762 \\ & & & 0.9986\end{array}$

Analysis of Variance

Source DF Sum of Squares Mean Square F Ratio

$\begin{array}{lllll}\text { Model } & 1 & 12.333086 & 12.3331 & 28200.19\end{array}$

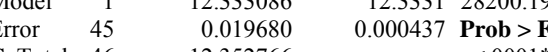

$\begin{array}{llr}\text { C. Total } 46 & 12.352766 & 0.00437 \\ <.0001 *\end{array}$

Parameter Estimates

Term Estimate Std Error t Ratio Prob $>\mid \mathbf{t}$

$\begin{array}{lrrrr}\text { Intercept } & 4.3704702 & 0.014156 & 308.74<.0001^{*} \\ \text { In(std wt\%) } & 1.0265697 & 0.006113 & 167.93 & <.0001^{*}\end{array}$ 
Exhibit A3. Linear Model of CU Values versus Natural Logarithm of Wt\% Solids Values

Bivariate Fit of CU By ln(std wt \%) Meter=System 2, Date=4-Dec, Position=Back

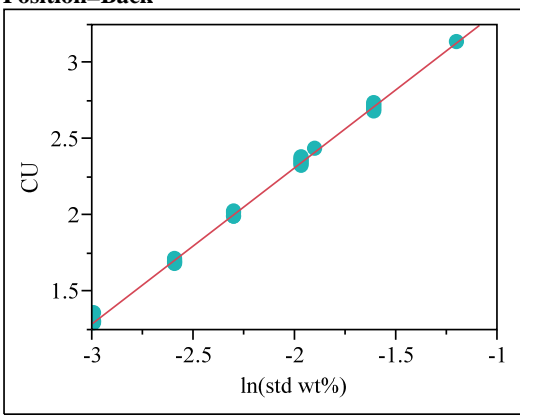

\section{Linear Fit}

Linear Fit

$\mathrm{CU}=4.3671137+1.0239563 * \ln (\mathrm{std} w \mathrm{w} \%)$

\section{Summary of Fit}

RSquare

RSquare Adj

0.998694

(O)

Observations (or Sum Wgts) $\quad 47$

\begin{tabular}{lrrrr}
\multicolumn{2}{l}{ Lack Of Fit } & & & \\
Source & DF & Sum of Squares & Mean Square & F Ratio \\
Lack Of Fit & 5 & 0.00322738 & 0.000645 & 2.0710 \\
Pure Error & 40 & 0.01246667 & 0.000312 & Prob $>$ F \\
Total Error & 45 & 0.01569405 & 0.0893 \\
& & & & Max RSq \\
& & & 0.9990
\end{tabular}

Analysis of Variance

Source DF Sum of Squares Mean Square F Ratio

$\begin{array}{llllll}\text { Model } & 1 & 12.270370 & 12.2704 & 35183.19\end{array}$

$\begin{array}{lrrr}\text { Error } & 45 & 0.015694 & 0.000349 \text { Prob }>\text { F }\end{array}$

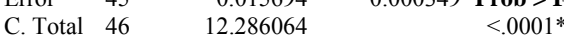

Parameter Estimates

Term Estimate Std Error t Ratio Prob $>|\mathbf{t}|$

$\begin{array}{llllll} & \text { Estercept } & 4.3671137 & 0.012641 & 345.47<.0001^{*}\end{array}$
Bivariate Fit of CU By ln(std wt \%) Meter=System 2, Date=4-Dec, Position=Frwrd

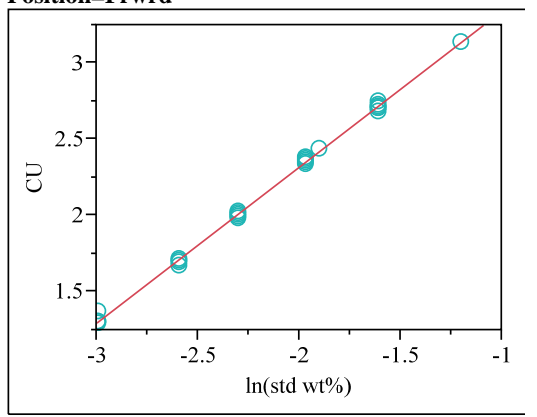

\section{Linear Fit}

Linear Fit

$\mathrm{CU}=4.367488+1.0234632 * \ln (\mathrm{std} \mathrm{wt} \%)$

\section{Summary of Fit}

RSquare

RSquare Adj

Root Mean Square

0.998512 2.053191

Observations (or Sum Wgts) $\quad 47$

\begin{tabular}{lrrrr}
\multicolumn{2}{l}{ Lack Of Fit } & & & \\
Source & DF & Sum of Squares & Mean Square & F Ratio \\
Lack Of Fit & 5 & 0.00342257 & 0.000685 & 1.8956 \\
Pure Error & 40 & 0.01444444 & 0.000361 & $\begin{array}{r}\text { Prob }>\text { F } \\
\text { Total Error }\end{array}$ \\
& 45 & 0.01786701 & 0.1167 \\
& & & & $\begin{array}{r}\text { Max RSq } \\
\text { RST } \\
\end{array}$ \\
& & & & 0.9988
\end{tabular}

Analysis of Variance
Source DF Sum of Squares Mean Square F Ratio

$\begin{array}{lrrrr}\text { Source } & \text { DF } & \text { Sum of Squares } & \text { Mean Square } & \text { F Ratio } \\ \text { Model } & 1 & 12.258554 & 12.2586 & 30874.50\end{array}$

$\begin{array}{lrrrrr}\text { Model } & 1 & 12.258554 & 12.2586 & 30874.50 \\ \text { Error } & 45 & 0.017867 & 0.000397 & \text { Prob }>\text { F } \\ \text { C. } & & & \end{array}$

\begin{tabular}{llrrr} 
Error & 45 & 0.017867 & 0.000397 & $\begin{array}{l}\text { Prob }>\text { F } \\
\text { C. Total }\end{array}$ \\
\hline & 46 & 12.276421 & $<.0001$ *
\end{tabular}

\section{Parameter Estimates}

Term Estimate Std Error t Ratio Prob $>|\mathbf{t}|$

$\begin{array}{lrlllll} & 4.367488 & 0.013488 & 323.81<.0001 *\end{array}$
Bivariate Fit of CU By In(std wt\%) Meter=System 2, Date=4-Dec, Position=Left

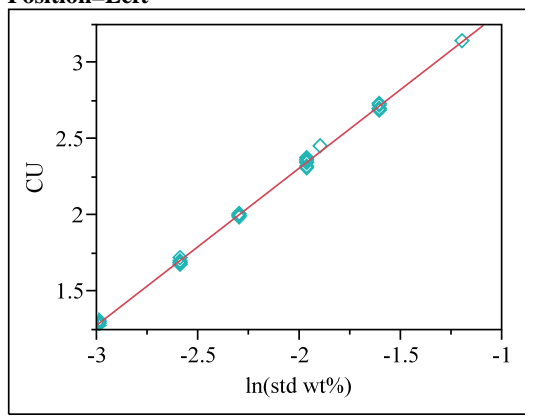

\section{- Linear Fit}

Linear Fit

$\mathrm{CU}=4.3772695+1.0300471 * \ln (\mathrm{std} \mathrm{wt} \%)$

Summary of Fit

RSquare

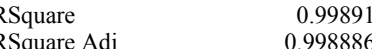

RSquare Adj $\quad 0.998886$

Root Mean Square Error $\quad 0.017351$

Mean of Response 2.048085

\section{Lack Of Fit}

Source DF Sum of Squares Mean Square F Ratio $\begin{array}{lrrrr}\text { Lack Of Fit } & 5 & 0.00192499 & 0.000385 & 1.3250\end{array}$ \begin{tabular}{lllll} 
Pure Error & 40 & 0.01162222 & 0.000291 & Prob $>$ F \\
\hline
\end{tabular}

$\begin{array}{llrr}\text { Total Error } & 45 & 0.01354721 & 0.2733 \\ & & & \text { Max RSq } \\ & & \end{array}$

Analysis of Variance

Source DF Sum of Squares Mean Square F Ratio

$\begin{array}{lrrrr}\text { Model } & 1 & 12.416780 & 12.4168 & 41245.03\end{array}$

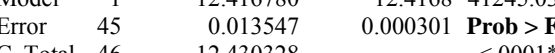

\section{Parameter Estimates}

Term Estimate Std Error t Ratio Prob $>|\mathbf{t}|$

$\begin{array}{llllll}\text { Intercept } & 4.3772695 & 0.011745 & 372.70<.0001^{*} \\ \text { In(std wt\%) } & 1.0300471 & 0.005072 & 203.09<.0001^{*}\end{array}$ 
Exhibit A3. Linear Model of CU Values versus Natural Logarithm of Wt\% Solids Values

Bivariate Fit of CU By ln(std wt\%) Meter=System 2, Date=4-Dec,

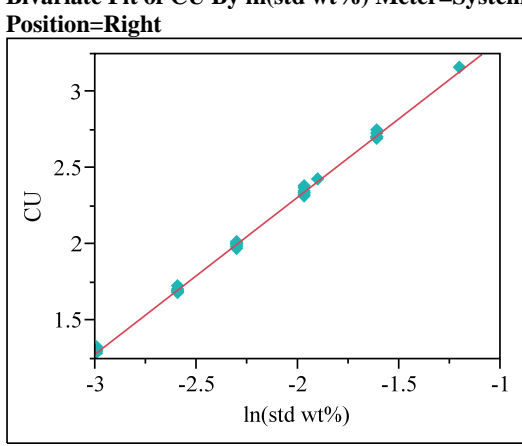

\section{— Linear Fit}

Linear Fit

$\mathrm{CU}=4.3745054+1.0293893 * \ln (\mathrm{std} \mathrm{wt} \%)$

\section{Summary of Fit}

\begin{tabular}{lr} 
RSquare & 0.998816 \\
RSquare Adj & 0.99879 \\
Root Mean Square Error & 0.018071 \\
Mean of Response & 2.046809 \\
\hline
\end{tabular}

Observations (or Sum Wgts)

Lack Of Fit

Source DF Sum of Squares Mean Square F Ratio

$\begin{array}{lllll}\text { Lack Of Fit } & 5 & 0.00213968 & 0.000428 & 1.3633\end{array}$

$\begin{array}{lll}\text { Pure Error } & 40 & 0.01255556\end{array}$

$\begin{array}{rrrr}\text { Total Error } & 45 & 0.01469524 & 0.2585 \\ & & \text { Max RSq }\end{array}$

Source DF Sum of Squares Mean Square F Ratio

$\begin{array}{lllll}\text { Model } & 1 & 12.400926 & 12.4009 & 37974.32\end{array}$

$\begin{array}{llll}\text { Error } & 45 & 0.014695 & 0.000327 \text { Prob }>\text { F }\end{array}$

C. Total $46 \quad 12.415621 \quad<.0001^{*}$

Parameter Estimates

Term Estimate Std Error t Ratio Prob $>|t|$

$\begin{array}{llllll}\text { Intercept } & 4.3745054 & 0.012232 & 357.62 & <.0001^{*} \\ \text { In(std wt\%) } & 1.0293893 & 0.005282 & 194.87 & <.0001^{*}\end{array}$ 
Exhibit A4. Statistical Investigation of LTL Values by Turbidity Meter

Distributions Meter=System 1 95/95 LTL

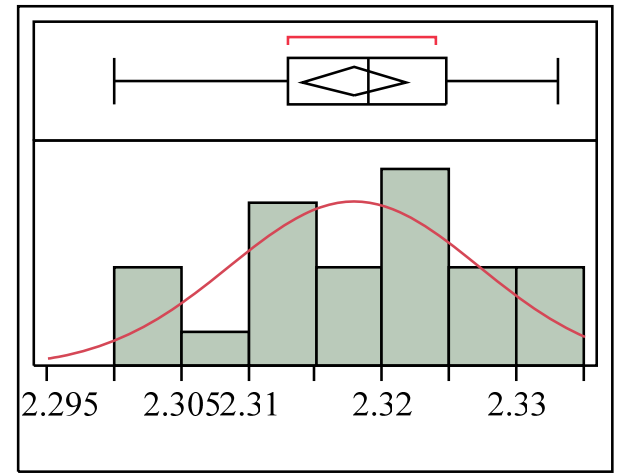

Normal(2.31779,0.0093)

Quantiles

$\begin{array}{llr}100.0 \% & \text { maximum } & 2.333 \\ 99.5 \% & & 2.333 \\ 97.5 \% & & 2.333 \\ 90.0 \% & & 2.33 \\ 75.0 \% & \text { quartile } & 2.32475 \\ 50.0 \% & \text { median } & 2.319 \\ 25.0 \% & \text { quartile } & 2.313 \\ 10.0 \% & & 2.301 \\ 2.5 \% & & 2.3 \\ 0.5 \% & & 2.3 \\ 0.0 \% & \text { minimum } & 2.3\end{array}$

Moments

Mean $\quad 2.3177917$

Std Dev $\quad 0.0093016$

Std Err Mean $\quad 0.0018987$

Upper 95\% Mean 2.3217194

Lower 95\% Mean 2.3138639

$\mathrm{N}$

Fitted Normal

Parameter Estimates

Type Parameter Estimate Lower 95\% Upper 95\%

$\begin{array}{lllll}\text { Location } \mu & 2.3177917 & 2.3138639 & 2.3217194\end{array}$

$\begin{array}{llll}\text { Dispersion } \sigma & 0.0093016 & 0.0072293 & 0.0130479\end{array}$

$-2 \log ($ Likelihood $)=-157.414209472197$

Goodness-of-Fit Test

Shapiro-Wilk W Test

$\begin{array}{rr}\mathbf{W} & \text { Prob }<\mathbf{W} \\ 0.952727 & 0.3101\end{array}$

Note: $\mathrm{Ho}=$ The data is from the Normal distribution. Small p-values reject $\mathrm{Ho}$

One-sided Tolerance Interval

Proportion Lower TI Upper TI 1-Alpha
$0.950 \quad 2.296312$
0.950

Distributions Meter=System 2

95/95 LTL

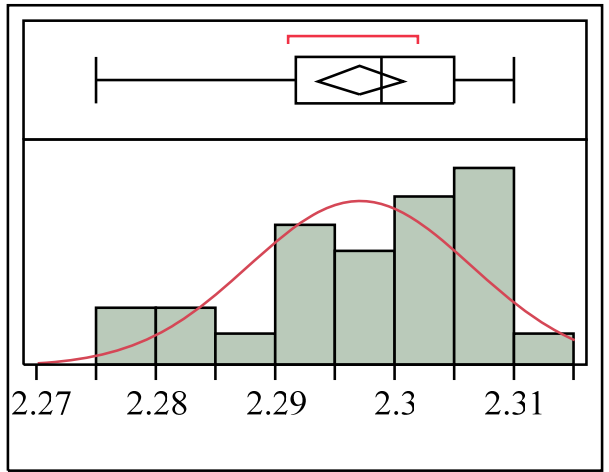

Normal(2.297,0.00933)

Quantiles

$\begin{array}{llr}100.0 \% & \text { maximum } & 2.31 \\ 99.5 \% & & 2.31 \\ 97.5 \% & & 2.31 \\ 90.0 \% & & 2.309 \\ 75.0 \% & \text { quartile } & 2.305 \\ 50.0 \% & \text { median } & 2.299 \\ 25.0 \% & \text { quartile } & 2.29175 \\ 10.0 \% & & 2.2824 \\ 2.5 \% & & 2.275 \\ 0.5 \% & & 2.275 \\ 0.0 \% \text { minimum } & 2.275 \\ \text { Moments } & \end{array}$

Mean 2.297

Std Dev $\quad 0.0093294$

Std Err Mean $\quad 0.0017631$

Upper 95\% Mean 2.3006175

Lower $95 \%$ Mean 2.2933825

$\mathrm{N}$

Fitted Normal

Parameter Estimates

Type Parameter Estimate Lower 95\% Upper 95\%

Location $\mu \quad 2.297 \quad 2.2933825 \quad 2.3006175$

$\begin{array}{llrr}\text { Dispersion } \sigma & 0.0093294 & 0.007376 & 0.0126985\end{array}$

$-2 \log ($ Likelihood $)=-183.316393011789$

Goodness-of-Fit Test

Shapiro-Wilk W Test

$\begin{array}{rr}\mathbf{W} & \mathbf{P r o b}<\mathbf{W} \\ 0.937403 & 0.0949\end{array}$

Note: $\mathrm{Ho}=$ The data is from the Normal distribution. Small p-values reject Ho.

One-sided Tolerance Interval

Proportion Lower TI Upper TI 1-Alpha

$\begin{array}{lll}0.950 & 2.276048 \quad . \quad 0.950\end{array}$ 
Exhibit A5. Plot of Time Study in Handling Sludge Simulant Standard Samples

CU By Time (sec) Wt $\%=0.05$ Wt $\%$

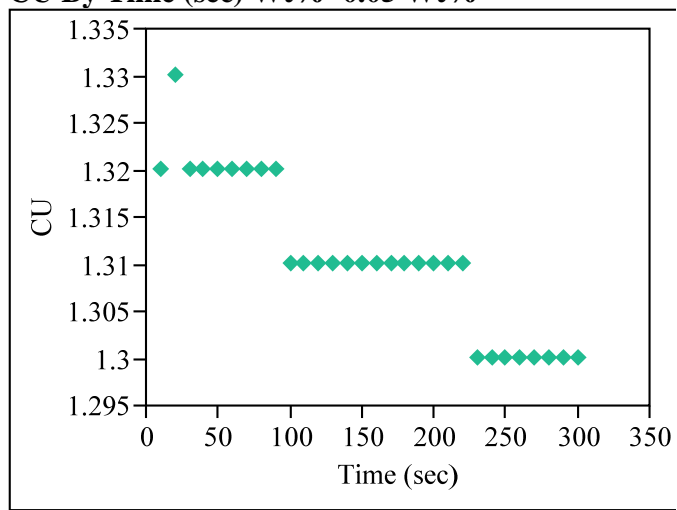

CU By Time (sec) Wt $\%=0.1 \mathrm{Wt} \%$

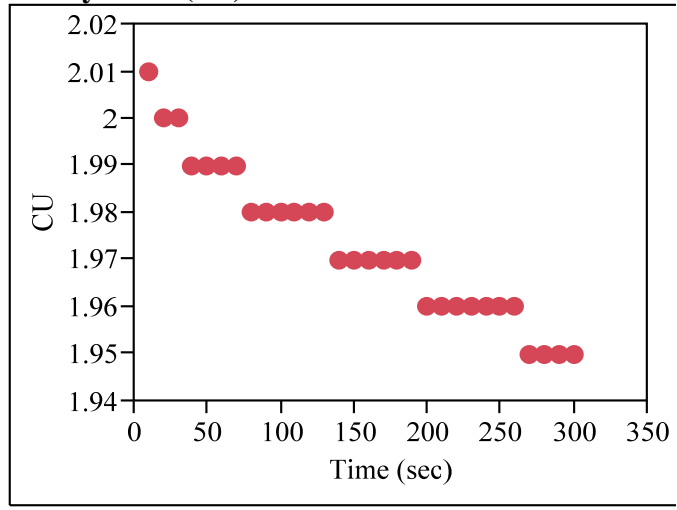

CU By Time (sec) Wt\%=0.15 Wt\%

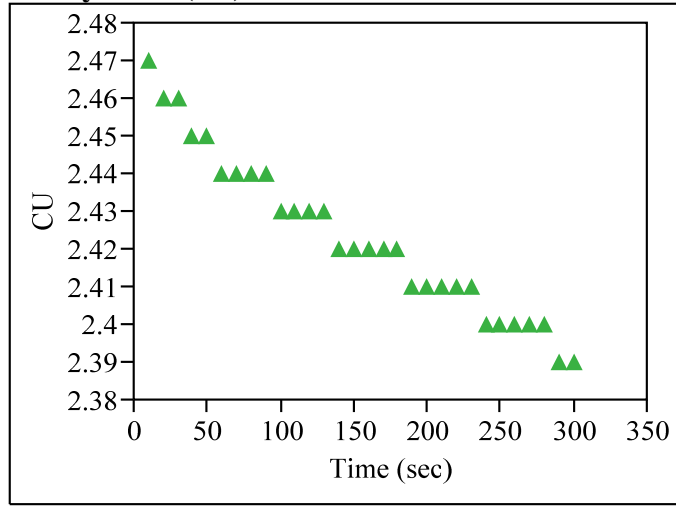

CU By Time (sec) Wt\% $=0.2 \mathrm{Wt} \%$

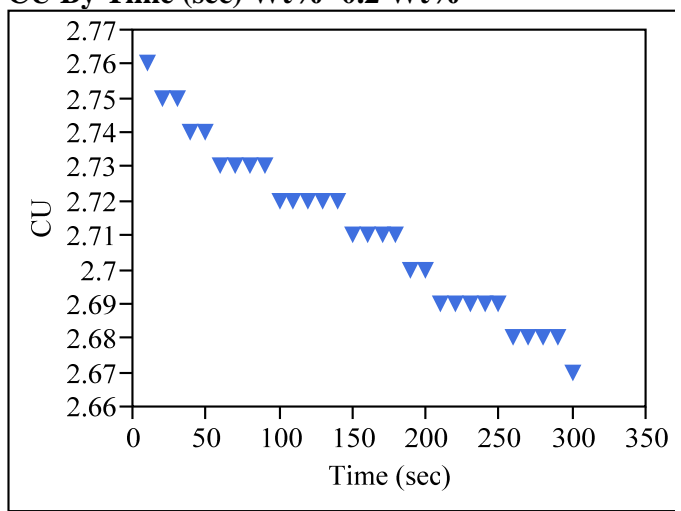

CU By Time (sec) Wt $\%=0.4$ Wt $\%$

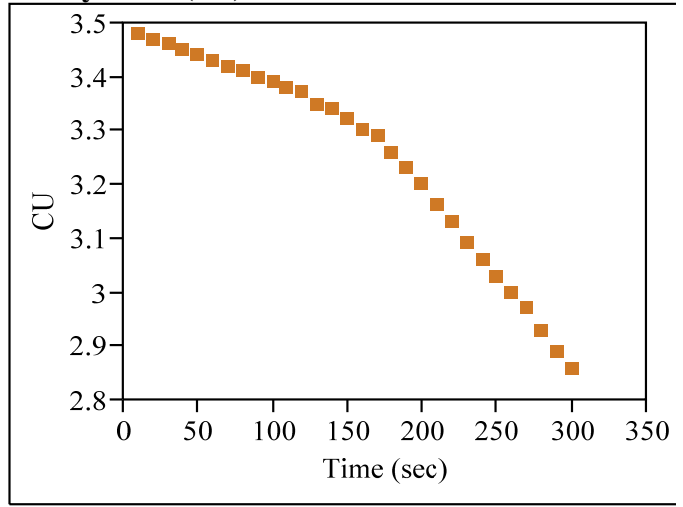

CU By Time (sec) Wt $\%=0.8 \mathrm{Wt} \%$

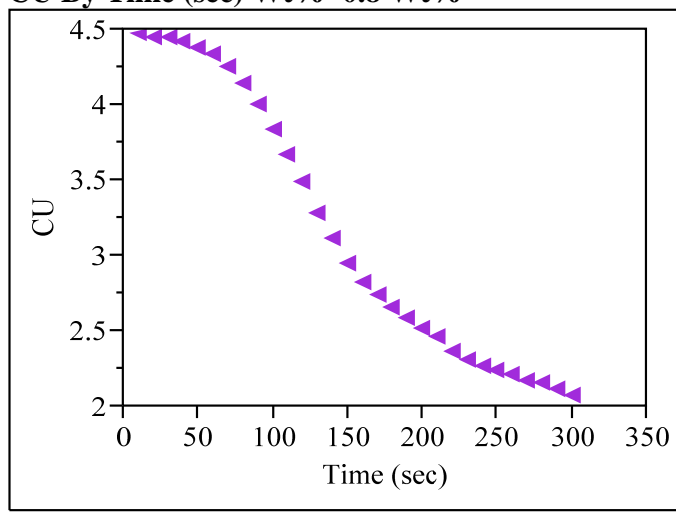


SRNL-STI-2013-00016

Revision 0

This page intentionally left blank. 


\section{Electronic Distribution by E-Mail to the Following}

\begin{tabular}{|c|c|}
\hline Name: & Location: \\
\hline Sharon Marra & 773-A \\
\hline Connie Herman & 999-W \\
\hline Patricia Lee & $703-41 \mathrm{~A}$ \\
\hline Gene Shine & $703-41 \mathrm{~A}$ \\
\hline Michael Stone & 999-W \\
\hline David Peeler & 999-W \\
\hline Tommy Edwards & 999-W \\
\hline Kevin Fox & 999-W \\
\hline Fabienne Johnson & 999-W \\
\hline Eric Freed & $704-S$ \\
\hline Jonathan Bricker & $704-27 \mathrm{~S}$ \\
\hline John Iaukea & $704-30 \mathrm{~S}$ \\
\hline Ryan McNew & $704-S$ \\
\hline Jeff Ray & $704-S$ \\
\hline Robert Hinds & $704-S$ \\
\hline Terri Fellinger & $704-26 \mathrm{~S}$ \\
\hline Michael J. Hart & $210-S$ \\
\hline Roger N. Mahannah & $704-28 \mathrm{~S}$ \\
\hline Michael T. Feller & $704-28 \mathrm{~S}$ \\
\hline Arthur Whittington & $704-28 \mathrm{~S}$ \\
\hline Paul Farrow & $704-26 \mathrm{~S}$ \\
\hline John Gregory & $704-S$ \\
\hline Amanda Shafer & $704-27 \mathrm{~S}$ \\
\hline Aria Behrouzi & $704-27 \mathrm{~S}$ \\
\hline Mason Clark & $704-27 \mathrm{~S}$ \\
\hline Helen Boyd & $704-27 \mathrm{~S}$ \\
\hline Hank Elder & $704-24 \mathrm{~S}$ \\
\hline Bill Holtzscheiter & $704-15 \mathrm{~S}$ \\
\hline Pat Vaughan & $773-41 \mathrm{~A}$ \\
\hline
\end{tabular}

